University of Louisville ThinkIR: The University of Louisville's Institutional Repository

Electronic Theses and Dissertations

$8-2017$

\title{
Perceptions of peer group interactions on self- efficacy and academic identity of African-American students in advanced placement classes.
}

Tinisha Yvette Taylor

University of Louisville

Follow this and additional works at: https://ir.library.louisville.edu/etd

Part of the Gifted Education Commons

\section{Recommended Citation}

Taylor, Tinisha Yvette, "Perceptions of peer group interactions on self-efficacy and academic identity of African-American students in advanced placement classes." (2017). Electronic Theses and Dissertations. Paper 2743.

https://doi.org/10.18297/etd/2743

This Doctoral Dissertation is brought to you for free and open access by ThinkIR: The University of Louisville's Institutional Repository. It has been accepted for inclusion in Electronic Theses and Dissertations by an authorized administrator of ThinkIR: The University of Louisville's Institutional Repository. This title appears here courtesy of the author, who has retained all other copyrights. For more information, please contact thinkir@louisville.edu. 


\title{
PERCEPTIONS OF PEER GROUP INTERACTIONS ON SELF-EFFICACY AND ACADEMIC IDENTITY OF AFRICAN-AMERICAN STUDENTS IN ADVANCED PLACEMENT CLASSES
}

\author{
By \\ Tinisha Yvette Taylor \\ B.A., University of Louisville, 2001 \\ M.A.T, University of Louisville 2005 \\ M.Ed., University of Louisville, 2010
A Dissertation
Submitted to the Faculty of the College of Education and Human Development
University of Louisville
for the Degree of

Doctor of Education in Educational Leadership and Organizational Development

Educational Leadership, Evaluation and Organizational Development University of Louisville Louisville, Kentucky

August, 2017 
Copyright 2017 by Tinisha Y. Taylor

All rights reserved 

PERCEPTIONS OF PEER GROUP INTERACTIONS ON SELF-EFFICACY AND ACADEMIC IDENTITY OF AFRICAN-AMERICAN STUDENTS IN ADVANCED PLACEMENT CLASSES

\author{
By
}

Tinisha Yvette Taylor

B.A., University of Louisville, 2001

M.A.T, University of Louisville 2005

M.Ed., University of Louisville, 2010

A Dissertation Approved on

July 12, 2017

By the following Dissertation Committee:

Dr. W. Kyle Ingle, Chair

Dr. Bradley Carpenter

Dr. Jason C. Immekus

Dr. Ann Larson 


\section{DEDICATION}

First giving honor to God above. Through Him all things are possible.

To my husband Dalton: thank you for your patience, motivation, love and support. You took care of our family and household while I wrote and wrote.....and wrote. You pushed me to keep going even I was exhausted and sleep deprived. Thank you for traveling this dissertation road along with me. You helped me to be resilient and to persevere. You are truly my rock! I love you.

To my daughter, Naya: I started this doctoral journey with you as my inspirationdetermined to show you that you too can be anything you want to be and that you can do whatever you put your mind to. For as long as you can remember, mommy has been in college working on one degree or another-well now I am finally finished! Your encouragement, love and support helped push me forward toward my goal. Now, I am looking forward to celebrating the beautiful young woman you will become and the opportunity to encourage and support you through life's journey wherever it may take you. You are priceless! I love you.

To Anthony: I know you do not understand much of what this degree means right now. You just see mommy writing all the time, too busy to spend much time with you. Just know that I am doing all of this in hopes to show you that through hard work and perseverance, you can achieve your wildest dreams and be whatever you want to be-if you only put your mind to it. You are amazing! I love you. 


\section{ACKNOWLEDGEMENTS}

I would like to thank my parents for instilling in me the guidance, wisdom and work ethic to persevere through adversity, for setting an example and for always challenging me to do more, be better and to never settle.

I would like to thank, my chair Dr. Ingle for his patience, patience and more patience--as well as his guidance and expertise throughout this doctoral journey. You have dedicated your time, energy, and a substantial amount of patience to assist me (and so many others) with this process. Your meticulous attention to detail helped to improve my skills as a researcher and writer. Your feedback and wisdom are invaluable and I have grown tremendously. Thank you for not giving up on us!

To Dr. Carpenter, thank you for the opportunity to participate in this program. Your enthusiasm for social justice drew us all in and kept us pushing for forward. Throughout this journey, I would say repeating inside my head "I must keep going I can't be 'ABD'." Thank you! 


\begin{abstract}
PERCEPTIONS OF PEER GROUP INTERACTIONS ON SELF-EFFICACY AND ACADEMIC IDENTITY OF AFRICAN-AMERICAN STUDENTS IN ADVANCED PLACEMENT CLASSES
\end{abstract}

\author{
Tinisha Y. Taylor
}

July 12, 2017

The last several decades have seen numerous efforts to close the achievement gap and minimize educational disparities between diverse student populations. This study explored the participants of one state-based initiative to increase the number of marginalized students (e.g., low socioeconomic status, students of color) in gifted classrooms. Research suggests that the educational experiences of gifted students of color is vastly complex, even multidimensional and is particularly influenced by sociocultural factors. For gifted African-American students these issues may be exacerbated as they struggle with identity development due to ostracizing from peers for their pursuit of academic excellence (Fordham \& Ogbu, 1986). The purpose of this study was to understand how African-American students who "self-identify" as gifted, perceive their peers as influencing the development of their own academic identity and efficacy beliefs. The study used student voice as a tool to capture the essence of informant experiences, allowing for a rich data collection and detailed cross-case analysis. 


\section{TABLE OF CONTENTS}

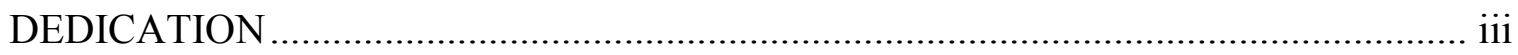

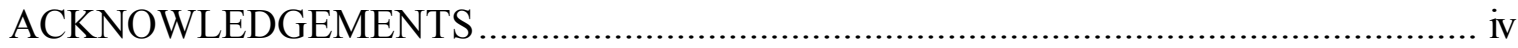

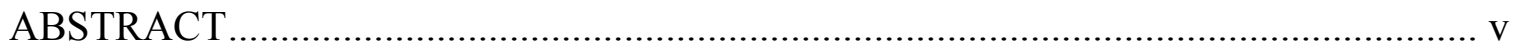

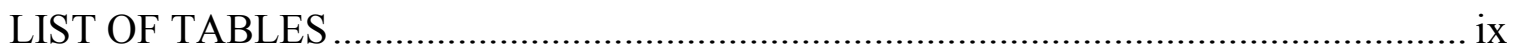

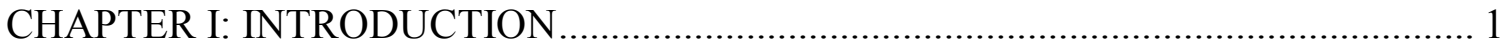

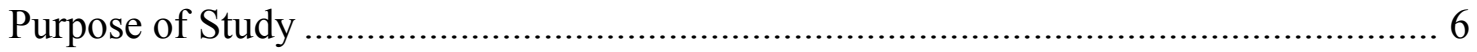

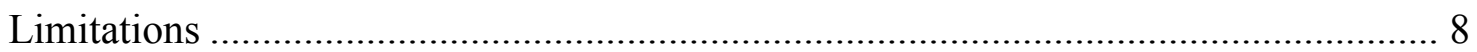

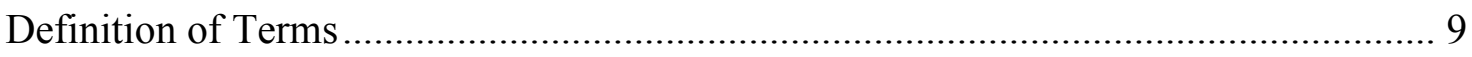

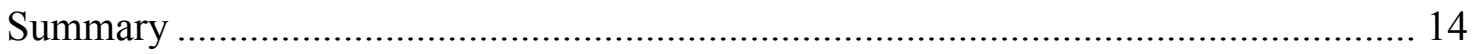

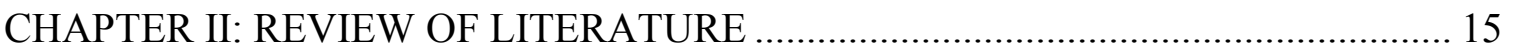

Academic Achievement among Students of Color and Reform Efforts ....................... 16

A Place for the Gifted: Advanced Placement............................................................... 21

Peer Group Experiences in Academic Contexts .......................................................... 30

Self-Efficacy Theory: Perception of Academic Ability …………………………....... 35

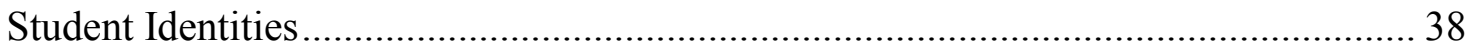

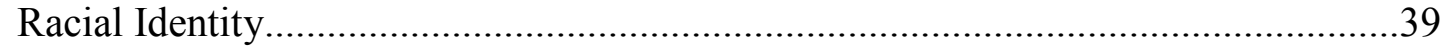

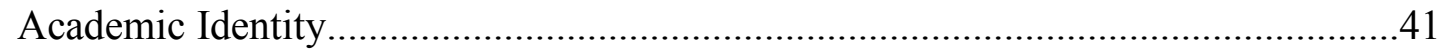

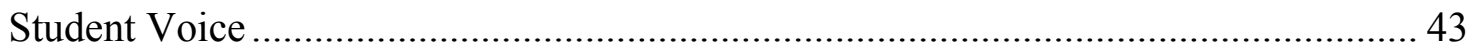

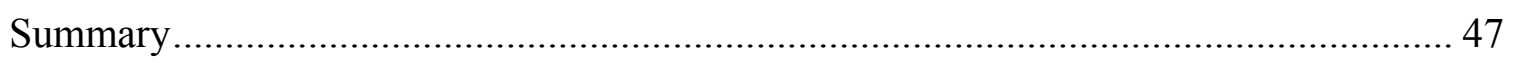

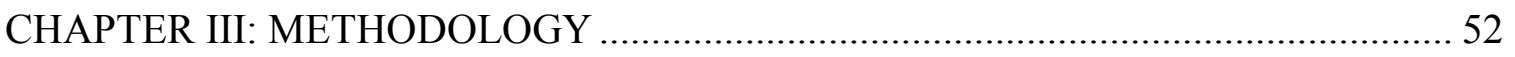

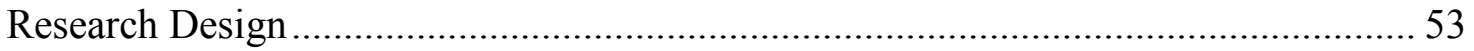

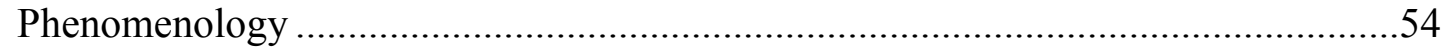

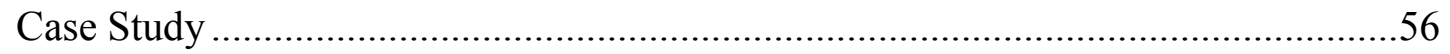

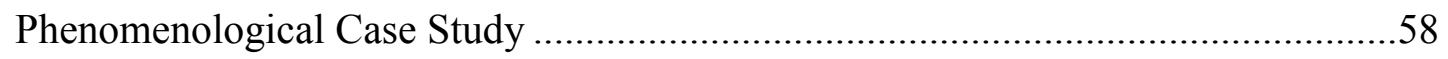

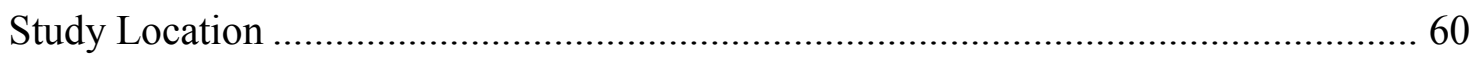

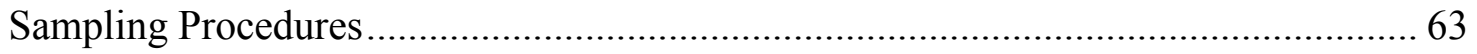

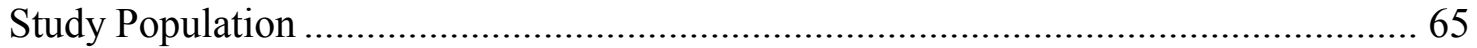

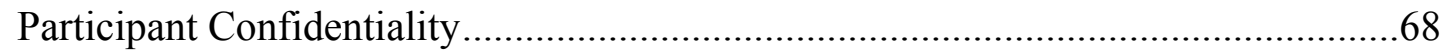

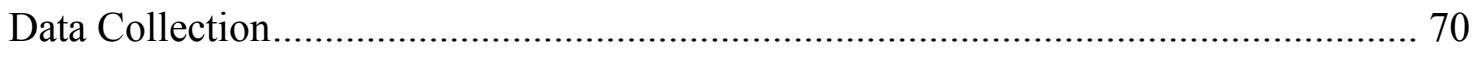




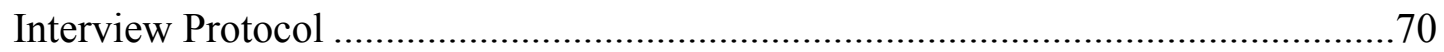

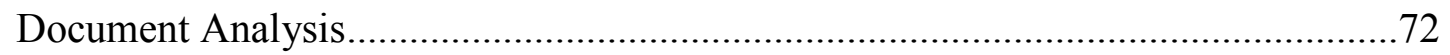

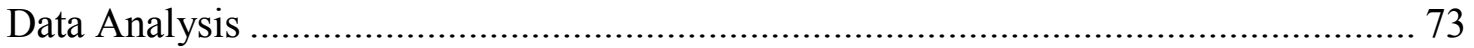

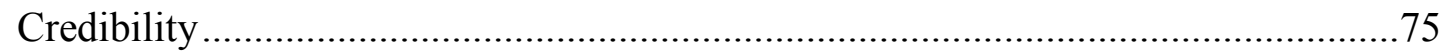

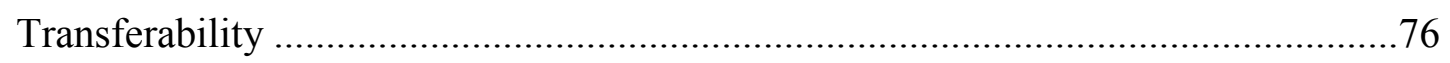

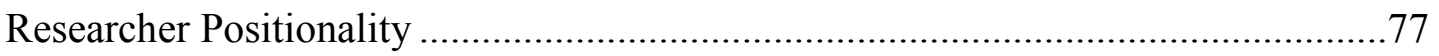

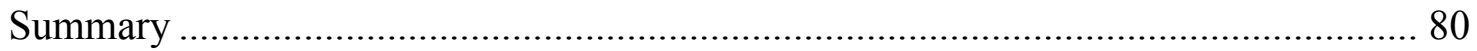

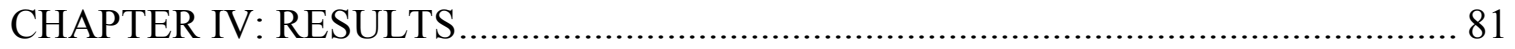

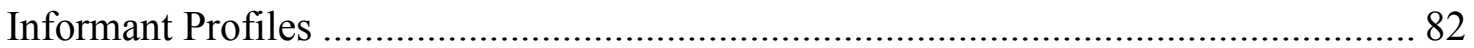

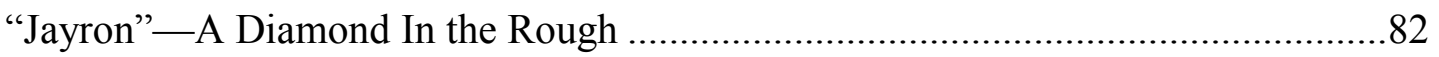

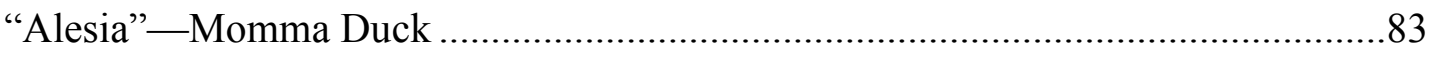

"Victoria"-I Can and I Will.................................................................................84

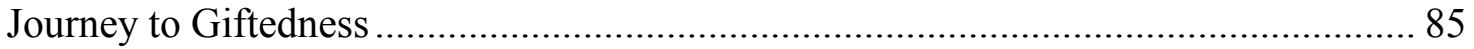

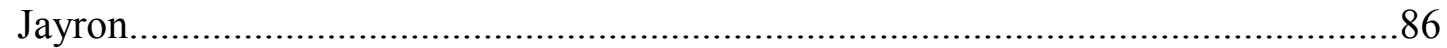

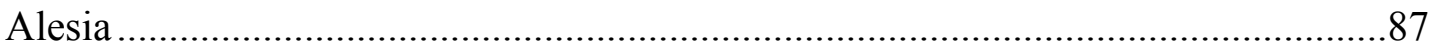

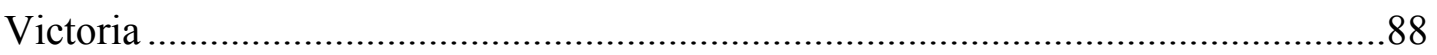

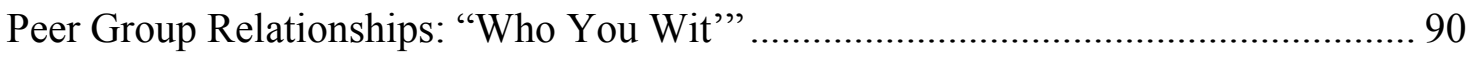

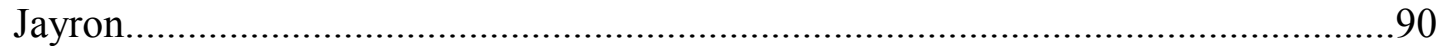

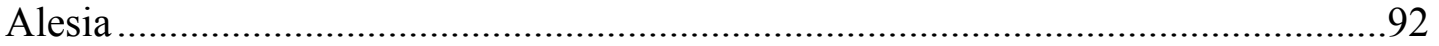

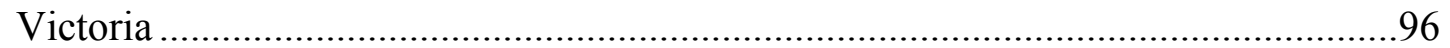

Self-Efficacy as a Gifted Student: "I Think I Can Do This"..........................................99

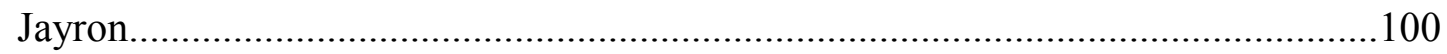

Alesia

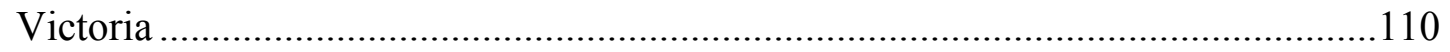

Academic Identity: African-American and Gifted.............................................. 116

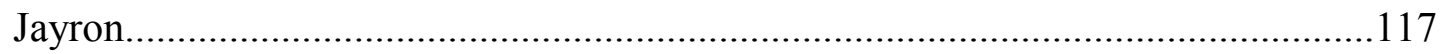

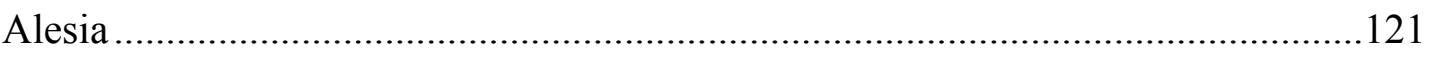

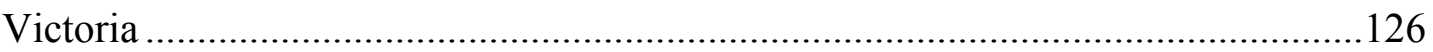

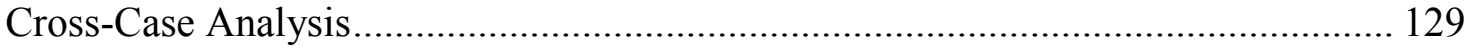

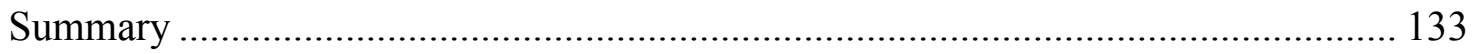

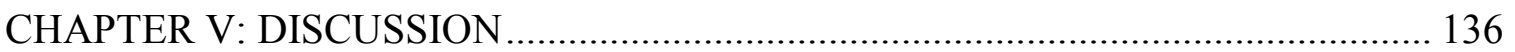

Peer Group Relationships................................................................................ 137 


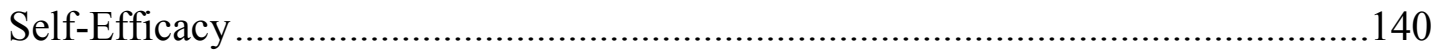

Academic Identity ..........................................................................................141

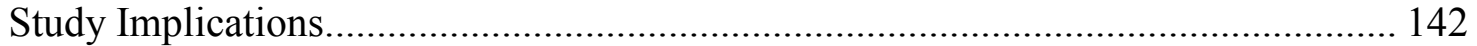

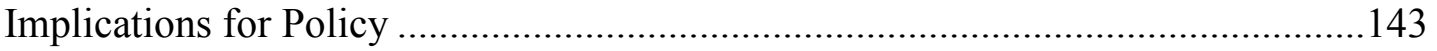

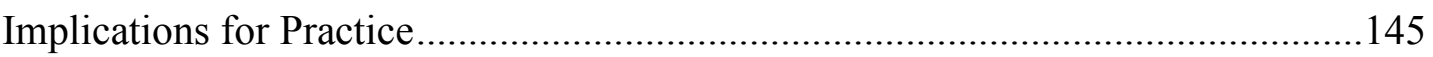

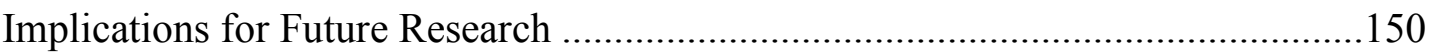

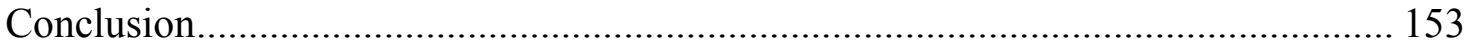

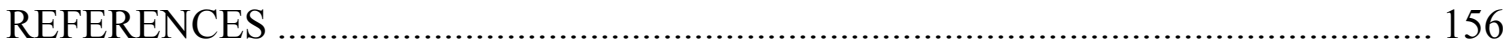

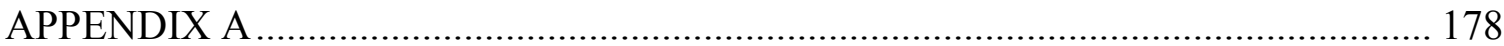

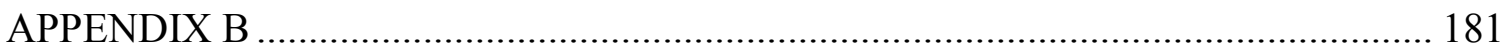

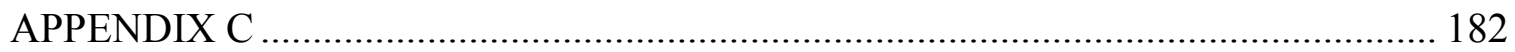

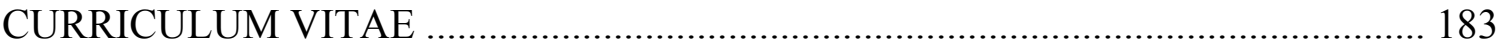




\section{LIST OF TABLES}

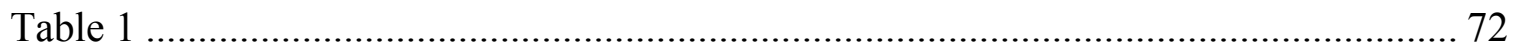

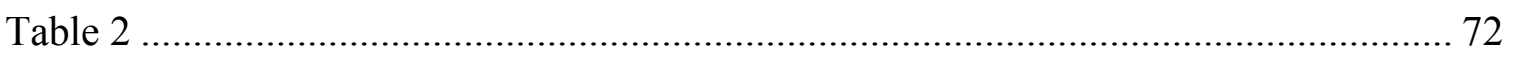

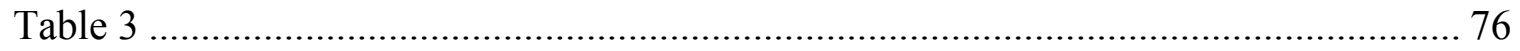

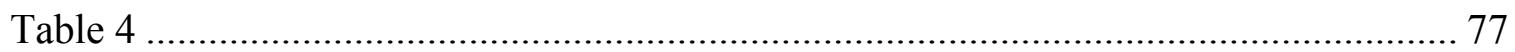

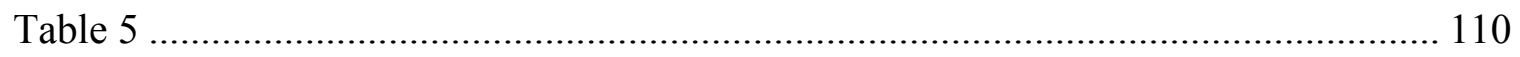

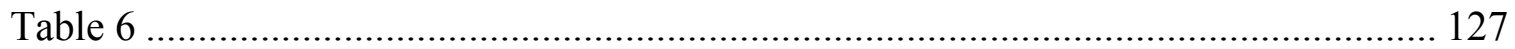

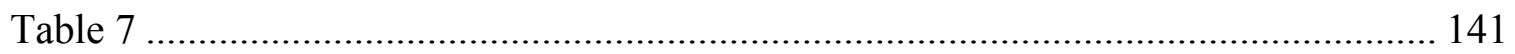

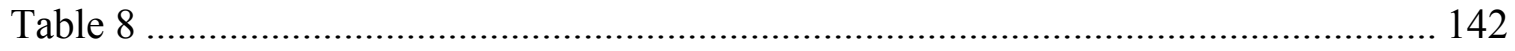




\section{CHAPTER I: INTRODUCTION}

The last several decades have marked a dramatic shift in racial demographics within the United States, marking a significant increase in the enrollments of racial and ethnic minorities in public schools (U.S. Department of Education, 2015). Despite enrollment growth, educational outcome disparities exist between students of color and their white counterparts. Results from the National Assessment of Educational Progress (NAEP) for eighth grade students indicated that on standardized assessments, an achievement gap of 31 points remained between African-American and White students (U.S. Department of Education, 2015). For other disenfranchised groups such as Latinos, assessment scores have gradually improved. However when placed alongside increased scores for White students, achievement gap data for Latinos remained relatively unchanged through 2009 (U.S. Department of Education, 2011). Researchers and policymakers offer various explanations and tentative solutions for the ongoing academic disparities of African-American students, yet they continued to lag behind White peers by as much as 30 points in some states (U.S. Department of Education, 2009a, 2009b, 2010).

In a concerted effort to decrease the achievement gap, the United States federal government enacted No Child Left Behind (NCLB) in 2002, the sixth reauthorization of the Elementary and Secondary Education Act of 1965 (ESEA). Among other things, the legislation required that states hold schools and districts accountable for improving graduation rates, college-career readiness and academic performance of student subpopulations based on race/ethnicity and program enrollment (e.g., special education). 
Further, NCLB emphasized uniformity for state academic standards, proficiency in literacy by the 2013-14 school year and maintained that schools report annual progress across identified student populations as measured by standardized assessments (ASCD, 2015). Despite its intentions and notable improvements in reading and math for some states, NCLB failed to address many of the inherent issues it set out to accomplish, including significant reduction to the achievement gap.

In December 2015, the U.S. Congress again reauthorized the Elementary and Secondary Education Act of 1965 (ESEA), replacing NCLB with the Every Student Succeeds Act (ESSA). The legislation sought to enhance improvements of educational outcomes for all students. In states and school districts where students persistently fall short of academic goals, ESSA insisted that in order to increase student rigor and accountability, educators must develop targeted interventions and supports to accommodate student needs (The White House, 2015). For urban schools serving large populations of marginalized students, achieving college and career readiness and increasing rigor are commendable goals that require educators be more than responsive to both the academic and nonacademic needs of students (Beckar \& Luthar, 2002; Gibson \& Dembo, 1984; Pajares, 1996). ESSA differs from NCLB by specifically addressing targeted objectives directed at improving state core content standards, assessments, accountability measures, educator effectiveness, comprehensive education programs and the allocation of federal funds. The legislation required that states' core content standards align with the acceptance criteria for post-secondary institutions and allowed state autonomy for the development of standards to accommodate students moderate to severe disabilities (ESEA, 2015). With regard to assessments, ESSA bears many similarities to 
NCLB with a few exceptions, including one that allows states the option to use a single annual exam or multiple tests given throughout the year resulting in a combined score.

Another noteworthy change in ESSA is in the determination of accountability for schools and states, specifically the elimination of NCLB yearly progress objectives and measures for subgroups, as well as proficiency goals. ESSA policies provide further autonomy for states to determine how much emphasis to place on accountability and the resulting penalties for schools who fall short. Additionally, ESSA requires a more detailed state issued school report card, including per pupil disbursements and higher education information (ESEA, 2015; ESSA, 2016; The White House, 2015). It outlined specific objectives to address improvements in the allocation of federal funds for persistently failing schools, teacher quality and effectiveness and ensured that schools receive resources for arts and physical education programs. With regard to school improvement, ESSA requires states to identify, closely monitor, and develop a clear rigorous plan for addressing low-preforming schools while doing away with federal grant incentives - forcing schools to allocate a percentage of their Title I funding towards school improvement needs. To address educator effectiveness, ESSA improved on NCLB's efforts to maintain qualified teachers and administrators by requiring states to prove that all federally funded schools are hiring employees that meet state certification requirements (ESSA, 2016). In order to enhance the quality of teachers and administrators, ESSA requires relevant professional development for all school-based educators that is directly linked to effectiveness particularly in under performing schools. Given the recent enactment of ESSA, it remains unclear how these changes will impact issues such as the achievement gap and diversity in gifted education. 
In response to the achievement gap, initiatives continue to emerge that seek to increase enrollments of students of color in gifted courses such as Advanced Placement (AP). Scholars report that African-American students are frequently overlooked as potential candidates for enrollment in gifted courses and that increasing their numbers may improve their academic performance and diminish the achievement gap (Baldwin, 2004; Kao \& Thompson, 2003; Klopfenstein, 2004; Kyburg, Herberg-Davis \& Callahan, 2007; Solorzano \& Ornelas, 2004; Zarate \& Pachon, 2006). In order to increase enrollments, particularly at low-performing urban high schools, some state and district administrators have adopted an alternative "open enrollment" process for admitting diverse groups of students into AP courses. Through open enrollment, underrepresented students (e.g., students of color, low socioeconomic status, English Language Learners) that are interested in enrolling in gifted courses may do so without testing qualifications. This reform effort was successful in increasing participation for students of color in AP courses, but the goal of closing the achievement gap has not been realized (Dougherty, Mellor \& Jian, 2006; Gubbins, Callahan \& Renzulli, 2014; U.S. Department of Education, 2010; The College Board, 2006). Increasing diversity within gifted programs merely scratched the surface of adequately preparing African-American students to matriculate successfully through college preparatory coursework and ultimately graduate from four-year post-secondary institutions (Gallagher, 2009; Lichten, 2007; The College Board, 2006; U.S. Department of Education, 2010). Although admirable, this initiative failed to address what Grantham and Ford (2003) describe as the social and cultural stigma encountered by academically successful students of color. 
Scholars recognize the modest increase in diversity among AP classrooms (Ford, 1998; Ford \& Thomas, 1997; Grantham \& Ford, 2003; Kao \& Thompson, 2003; Klopfenstein, 2004; Kyburg et al., 2007; Ndura, Robinson \& Ochs, 2003), but contend that increasing enrollment alone is inadequate in addressing the real problem - lower levels of academic achievement in gifted courses. Among African-American students taking AP examinations, $72 \%$ scored below a three, which is the minimum score required to receive college credit (Kyburg et al., 2007; The College Board, 2006). Lichten (2007) argues that simply placing minorities in AP courses is not enough and that effective preparation and supports are needed to ensure they are successful. Gifted courses add a unique environmental component to the pluralistic academic experience of students of color (Horvat \& Lewis, 2003), which are also influenced by social and cultural nuances that help to shape their identity. Baldwin (2004) notes that minority students have academic experiences that are distinct from their White peers and require targeted supports that embrace their unique needs. In their assessment of high-performing minority students, Ford and Grantham (2003) note that these students are laden with socioemotional and psychological stress stemming from their minority peers' perceptions that they have disconnected from cultural norms by "acting White." In her review of culturally diverse gifted populations, Baldwin (2004) contends that in order to expand access and offer effective support, educators must rethink their attitudes and preconceived notions about acknowledging academic potential in students of color.

Despite a slight increase, researchers contend both academic and non-academic factors contribute to the lack of diverse enrollment in gifted and talented courses, yet they agree in the need for further action in order to address what Ford (1998) candidly refers 
to as "the persistent and pervasive under-representation of minority students..." (p.12) and what Kao and Thompson (2003) insist is a "racial and ethnic disadvantage" (p.423). Several studies have examined the quantitative effects of peer influence on student academic achievement by comparing social interactions among grade-level peers at the elementary and secondary levels, finding that these factors may have adverse effects on student learning (Guay, Boivin \& Hodges, 1999; Nelson \& Debacker, 2008; Ryan, 2000; Schunk \& Pajares, 2001; Zimmerman, 2003). Educators and researchers continue to search for solutions and the answer lies beyond those perceived deficit characteristics commonly recognized as the cause for the marginalization of gifted students of color (Klopfenstein, 2004). Perhaps a more effective method to ascertain those factors influencing achievement would be to ask the students themselves. Mitra (2004) praises student voice as a valuable approach to research, stating:

...students possess unique knowledge and perspectives about their schools that adults cannot fully replicate. Through open conversations about injustices in schools, student voice can raise issues of equity that tend to get swept under the rug by administrators and other adults in the school who would rather avoid controversy. (p.652 - 653)

Research that captures the voices of high-achieving minority students, the sociocultural nuance of their powerful personal experiences, celebrations, concerns, stressors, and hindrances may inform the response and supports that educational leaders, teachers, and support staff can provide these students. 


\section{Purpose of Study}

As previously discussed, researchers suggest that for students of color, environmental elements such as socioeconomic status, quality of schools, teacher efficacy, racism and parental education attainment influence their participation and achievement in school (Kao \& Thompson, 2003; Klopfenstein, 2004; Kyburg et al., 2007; Solorzano \& Ornelas, 2004). Among adolescents, the dynamics of peer relationships are thought to play significant roles in social behavior, self-efficacy, and perceptions of academic ability and those experiencing pressures from peer groups may have their academic behavior patterns and perceptions of ability reinforced. The extant literature presents contrasting arguments, identifying multiple factors that contribute to low achievement for both gifted and non-gifted students of color. A barrage of disheartening assessment statistics, coupled with the under-representation of students of color in advanced level classes, elicit the need for alternative approaches to the problem.

Given that networking among peer groups was found to influence feelings of academic self-efficacy, as well as impinge upon their academic decisions (Schunk \& Pajares, 2001), capturing the unique experiences of gifted minorities and their interaction with peers may provide insights into what encourages and hinders their achievement. Horvat and Lewis (2003) challenge Fordham and Ogbu's (1986) theory that black students view their successful peers as acting White. They suggest that this perception is "oversimplified, leading many to overlook the collaboration and culmination of multiple forces on the educational outcomes of the black students" (Horvat \& Lewis, 2003, p.266). In his analysis of peer effects on achievement, Zimmerman (2003) maintains that quantifying peer effects is a challenging task, amplifying the need for qualitative study to 
discover the inherent characteristics of social relationships among peers groups and how these interactions might inspire or hinder achievement. Moreover, additional research is needed to uncover alternative explanations for the under-representation and underachievement of gifted students of color. For example Ford (1998) states that, "to avoid reaching erroneous conclusions about the minority under-representation problem, we must look not only at the changes in figures, but also at contextual factors - both sociocultural and sociopolitical" (p.11). Exploring the unique sociocultural contextual factors surrounding gifted students of color through the use of student voice helped uncover influences on self-efficacy and academic identity and provide insight for educators to create holistic support systems to ensure student success.

The purpose of my study was to explore the perceptions of urban AfricanAmerican students in AP courses. I sought specifically to understand how they perceived peers as influencing the development of their own academic identity, as well as the beliefs about their own academic potential (e.g., self-efficacy). Guiding my study were the following research questions:

1. What are the perceived influences of peers on the development of self-efficacy and academic identity of African-American students enrolled in Advanced Placement courses?

2. How do African-American students in Advance Placement courses perceive interactions with peers and cultural ideas towards achievement, as influencing their overall academic experiences in Advance Placement courses?

Through this study, I sought to contribute a unique qualitative perspective, giving voice to a marginalized population of students. Providing a platform for gifted minorities 
to express their perceptions and concerns would assist high school educators in developing support systems aimed at addressing the social and academic needs of these students. My study focused on three African-American students, all of whom participated in an open enrollment program to attend at least one AP course at their high school. These students attend the same high school, which is part of a large urban school district located in the southeastern United States. In an attempt to uncover themes, I also solicited perspectives from one peer group member per gifted minority student that I interviewed, as well as an in-school trusted adult (e.g., teacher, coach, and/or mentor). I discuss each of these informants in more detail in Chapter III.

Two theoretical frameworks guided my study - a multifaceted interpretation of academic identity, as it pertains to interactions among peer groups of highly motivated and successful minority students and Bandura's (1977) theory of self-efficacy in academic settings. Horvat and Lewis (2003) note that for gifted minority students, academic identity is "multidimensional" and "requires and understanding of how students manage their success," as well as the "various orientations of the peer groups" (p.276). By exploring the sociocultural academic experiences of gifted African-American students in relation to their peers, I gained insight into experiences that could help shape best practices for AP educators and stakeholders, allowing them to better address needs of this marginalized population.

\section{Limitations}

I must acknowledge several limitations of my study. Although not generalizable beyond the nine student-informants (three gifted African-American students, three peers, and three trusted adults), my research brought awareness to the personal experiences of 
gifted African-American students, thus providing educators with insight for improving best practices. My study did not explore the experiences of gifted minority students from other racial subpopulations. Asian Americans, Native-Americans and Pacific Islanders were excluded due to the fact that their numbers total less than $2 \%$ of the student population for the sampled district and school site. Given the above parameters I recognized the intent of this case study research design was "not to represent the world, but to represent the case" (Stake, 2005, p. 460).

The depth and honesty of the qualitative data that I collected from the informants depended on my ability to make them comfortable in discussing their personal experiences with me. I took concrete steps to ensure that the informants were fully informed of their rights as voluntary participants, as explained in the consent forms. I also disclosed my positionality to establish rapport and integrity. Beyond its limitations, my study has relevance and implications for urban educators seeking to mitigate the achievement gap through diverse participation in gifted programs, specifically using open enrollment (i.e., self-nomination) protocol. It is incumbent upon educational leaders and educators to listen to the voices of the students they serve in order to understand how to implement holistic support systems that meet their needs.

\section{Definition of Terms}

The following definitions of terms were used in the context of my study:

Academic Identity: There is no concise definition for academic identity in the literature, however it is best described as a multidimensional process by which an individual defines oneself within an educational context. In her study of academic success in gifted Puerto Rican students, Flores-Gonzalez (1999) describes academic identity as "the interaction of 
factors such as minority status, social class, sociocultural context of the school, and ethnic identities" (p. 346). Thus, in the context of this study, Flores-Gonzalez's (1999) interpretation provides a multifaceted view of academic identity which is applicable to marginalized groups of students attempting to navigate the interconnected nuances of their ethnicities, peer groups and culture, all while trying to maintain academic success. Advanced Placement (AP): For many schools, Advance Placement classes are designated as the primary setting for gifted and talented students. The AP program as a challenging academic program offered at more than 19,000 high schools nationwide designed to prepare high school students for college level coursework by providing the potential to earn college credit upon passing end of course examinations (The College Board, 2015).

AdvanceState (pseudonym) ${ }^{1}$ : AdvanceState is a statewide open-enrollment initiative aimed at increasing opportunity and access to gifted programming (e.g., Advanced Placement) for underrepresented student populations without traditional means of testing in. Students who express interest in gifted courses me voluntarily enroll and receive funding to offset the cost of cumulative examinations. A specific goal of AdvanceState was to increase accessibility to post-secondary education for students of color by arming them with the critical thinking skills needed to prepare them for the rigorous coursework. In addition, instructors receive professional development to assist with content-based training. State administrative data revealed that by 2013-14 school year 100 high schools across 74 districts adopted AdvanceState open enrollment protocols.

\footnotetext{
${ }^{1}$ AdvanceState is a pseudonym used to protect the State organization tasked with coordinating access to gifted programming for nontraditional students.
} 
Gifted and Talented: Throughout literature, the term "gifted and talented" is used to identify students with inherent potential to achieve academic success. The U.S. Department of Education has modified Marland's (1971) definition ${ }^{2}$ to include diverse groups of students, indicating "outstanding talents are present in children and youth from all cultural groups, across all economic strata, and in all areas of human endeavor" (Ross, 1993, p. 26). According to the National Association for Gifted Children (2015b), gifted courses are defined as “...education programs that will challenge high-ability learners [them] in regular classroom settings and enrichment and accelerated programs to enable them to make continuous progress in school."

Gifted Programs: By this definition, given their rigorous academic design and in the context of this study, The College Board Advanced Placement courses are tagged as qualifiers for gifted education. Given the parameters of this study the terms "traditional enrollment" and "open enrollment" refer to AP admission processes in which students are required to test-in or self-nominate to participate respectively.

Self-Efficacy: A theory developed by Albert Bandura during the 1970s, self-efficacy is rooted in human behavior interactions and defines an individual's belief in one' own abilities. Bandura (1993) pinpoints cognitive attributes such as aspirations, enthusiasm and academic success that are influential in determining individual self-efficacy. There are inherent differences in self-efficacy with regard to ability, gender and race/ethnicity however, a student's ability to gauge their inherent capability to complete a task or reach a goal is vital in helping to shape their academic success. Self-efficacy is a basic function

\footnotetext{
2 "Gifted and talented children are those identified by professionally qualified persons who by virtue of outstanding abilities, are capable of high performance," (Marland, 1971, p.8).
} 
of cognitive development and therefore influences a child's belief in their ability to master challenging curriculum (Bandura, 1993).

Sociocultural: Among racial and ethnic minorities there are recognized social differences that are generally associated with specific groups. For example, individuals from AfricanAmerican and Latino subpopulations often struggle to overcome socioeconomic barriers which result in culminating disparities that affect everything from education to housing (Betancourt, Green, Carrillo, \& Ananeh-Firempong, 2003). These racial subgroups also have distinct cultural features that define their relationships with community and family and influence their perspectives on society, politics and interactions both within and outside the group. Betancourt and his colleagues (2003) describe sociocultural as a contextual component resulting from a strategic understanding of cultural competency and socio-economic factors that affect a particular group. For the purpose of this study, sociocultural refers to the social (e.g., peer relationships) and cultural features (e.g., family, community) that shape the academic and self-efficacy beliefs of gifted AfricanAmerican students.

Students of Color: As previously discussed in the context of this study the terms "minorities" and "students of color" refers to African-American students exclusively, due to their representative numbers within the sampled district. For this study gifted minorities/students of color included student who is identified as a minority, as defined above in the context of this study (e.g., African-American) and enrolled in an at least one AP course via open enrollment.

Student Voice: The idea of student voice was initiated during the 1960s and 1970s when student demonstration became a prominent means of educational protest. Student voice 
includes recognizing students as viable stakeholders in their educational process and considering their opinions, thoughts and perspectives as essential to improving schools (Mitra, 2004; Mitra, Serriere \& Stoicovy, 2012). More recently, student voice has resurfaced with the educational reform efforts aimed at improving academic outcomes for students. Student voice honors integrity and the principle that their voices matter and should be part of the decision-making process. Scholars contend that students have unique perspectives and experiences that cannot be accurately portrayed by adults (Fielding, 2004b; Fielding \& Rudduck, 2004; Mitra, 2004; Mitra et al., 2012).

Open enrollment: In an effort to increase enrollment for underrepresented student populations, some state and district administrators decided to diminish barriers associated with restricted access to gifted courses. Scholars recommended using alternative methods for assessing students' eligibility for gifted programs with particular concern about enrollment opportunities for African-American students (Ford, 1998; Frasier, Garcia \& Passow, 1995). Currently, many school districts have adopted policies which allow for disadvantaged students (e.g., low socioeconomic, students of color) to voluntarily enroll into advanced course with testing-in. Gallagher (2009) notes that when compared to traditional enrollment practices (e.g., testing-in), schools participating in alternative placement processes seen increased student diversity in gifted programs.

Traditional enrollment: Traditionally, students are identified as qualified to enroll in gifted programs, such as Advanced Placement, based on standardized assessment results. The College Board determined that students should be identified for giftedness based on their performance on the standardized assessments, such as the Scholastic Aptitude Test (Chenoweth, 1998; Klopfenstein, 2004) coupled with district-defined abilities (Ford \& 
Thomas, 1997). Selecting gifted students became the primary method for entry to Advanced Placement courses.

\section{Summary}

I divide my study into five chapters outlining the knowledge base, procedures, protocols, results, discussion and implications. This introductory chapter is followed by Chapter II, which reviews literature highlighting historical perspectives, existing studies using relevant frameworks and models for exploring the academic experiences of minority students, self-efficacy beliefs and factors influencing the formation of academic identities. Chapter III discusses my selection of qualitative methodology, data collection and analysis techniques for the proposed study. Chapter IV presents study findings from the nine informant that I interviewed for this study as well a document analysis and results of cyclic coding within categories determined by theoretical frameworks. I conclude with Chapter V, a summary of findings and the implications for stakeholders, policy, and recommendations for future research. 


\section{CHAPTER II: REVIEW OF LITERATURE}

In this study, I sought to explore the sociocultural academic experiences of gifted African-American students, who elect to enroll in Advanced Placement (AP) courses through an open enrollment process offered at their urban high school. In particular, I was interested in how peer group relationships shape gifted minorities' efficacious beliefs in terms of academic ability, which in turn may influence how they establish their academic identity. I answered the following research questions: (1) What are the perceived influences of peers on the development of self-efficacy and academic identity of AfricanAmerican students enrolled in AP courses? (2) How do African-American students in Advance Placement courses perceive interactions with peers and cultural ideas towards achievement, as influencing their overall academic experiences in Advance Placement courses?

To demonstrate need for the proposed study and its research questions, my review of literature begins with a discussion of academic achievement as measured by annual standardized tests used for accountability, as well as reform efforts aimed at closing the African-American-White achievement gaps, in particular initiatives aimed at increasing enrollment in Advance Placement courses. Next, I provide a brief history of Advance Placement program, AP minority enrollment trends and effectives. Finally, I discuss literature on the social constructs of peer relationships among students of color, as well as theoretical frameworks of self-efficacy and academic identity. Also included is a 
discussion of student voice, utilized as a tool for capturing realistic informant experiences.

\section{Academic Achievement among Students of Color and Reform Efforts}

Since the enactment of No Child Left Behind in 2002, educational reform efforts have focused on increasing assessment scores of minority students in order to address substantial gaps in achievement. According to the National Center for Education Statistics (2009a, 2009b), the African-American-White achievement gaps have fluctuated over the last 30 years. However, significant disparities remain across standardized reading and math assessments. In some instances for students of color scores on the National Assessment of Educational Progress show significant deficits in comparison to their White counterparts (U.S. Department of Education, 2009a, 2009b). Scholars assign several contributing factors to this identified gap, poverty (Brooks-Gunn \& Duncan, 1997) including low scores on standardized assessments (Kober, 2001), high grade level retention rates and frequent assignment to low-performing academic groups (e.g. special education) (Beckar \& Luthar, 2002). In her discussion of inequity, Ladson-Billings (2006) describes the achievement gap as “... a way of explaining and understanding the persistent inequality that exists (and has always existed) in our nation's schools" (p. 5). Many of the schools she refers to are designated as persistently low achieving or "failing" and are required by state and federal policy to meet benchmarks designed to demonstrate growth in student achievement, which in turn is their contribution to reducing the achievement gap. Yet continuous low performance coupled with the challenges that accompany their impoverished communities, educators in urban schools were working 
tirelessly to reach state and federal NCLB accountability measures (Becker \& Luthar, 2002; Lee \& Bowen, 2006).

As part of the recently enacted Every Student Succeeds Act (ESSA), Congress decided to revise NCLB provisions to streamline accountability measures for schools struggling to improve student achievement. As part of a 2015 press release overview of ESSA, Press Secretary Josh Earnest stated "the bill rejects the overuse of standardized tests and one-size-fits-all mandates on our schools, ensures that our education system will prepare every child to graduate from high school ready for college and careers..." (The White House, 2015, p. 1). Two important provisions of ESSA included improving college and career-readiness (CCR) standards and increasing rigorous accountability for every child. These CCR revisions focused on the implementation of President Obama's 2008 Race to the Top initiative which provided states with financial incentives for improvements in academic outcomes. Although the effectiveness of ESSA policies on the achievement gap remains to be seen, its focus on diverse educational opportunities for all students in promising.

The harsh reality of the achievement gap became a central component of educational research, as scholars investigated its causes and theorized practical solutions for educators, parents and communities to impede its progressive growth (Beckar \& Luthar, 2002; Ladson-Billings, 2006; Lee \& Bowen, 2006; Viadero \& Johnston, 2001). Academic challenges among disadvantaged populations have been attributed to embedded cultural deficiencies, such as poverty, parenting styles, cultural-family dynamics and education values, which may prevent students' ability to learn in context of mainstream schools (Kober, 2001; Ladson-Billings, 2006; Lee \& Bowen, 2006). Thus, 
within the literature there was no single source credited with causing the achievement gap. For African-American students, biases in curriculum and testing (Kober, 2001; Viadero \& Johnston, 2000), ineffective pedagogical methods (Kober, 2001; LadsonBillings, 1999, 2006), poverty (Brooks-Gunn \& Duncan, 1997, Haveman \& Wolfe, 1995; Kober, 2001; Lee \& Bowen, 2006), social-emotional factors (Beckar \& Luthar, 2002), as well as cultural and family dynamics (Kober, 2001, Ladson-Billings, 1999) were all identified as contributing factors to lags in achievement.

For years test bias has been linked to academic achievement among students of color. Viadero and Johnston (2000) recognized inherent test bias against minority groups as a factor influencing the achievement gap. In her analysis of the achievement gap, Kober (2001) identified bias questioning within standardized tests as a reason for lags in achievement among students of color and called for "stricter accountability systems that monitor subgroup performance" (p. 27). In their discussion of the Black-White achievement gap, Jencks and Phillips (2011) explicitly outlined several types of racial bias in standardized testing and the impacts of each on achievement outcomes. Selection system bias, which is considered an important of racial testing, occurs under the appearance of performance conditions that are designed to measure cognitive skills without regard to racial and cultural factors that influence performance (Jencks \& Phillips, 2011). For educational institutions selection system bias results in test scores that may be an inaccurate measure of student skills and "...puts Blacks and Hispanics at a greater disadvantage than a selection system based on actual performance" (Jencks \& Phillips, 2001, p.58). Testing bias may be an influential factor affecting academic 
outcomes for African-American students, yet it alone cannot explain vast differences in the achievement gap.

Ladson-Billings (1999) also acknowledged test bias as an influential factor on the achievement gap, but insisted that pedagogical practices were direct source of inequity in education. She argued that this is a result of teachers being unprepared to effectively communicate with and address the learning needs of diverse groups of students in their classrooms. Throughout the article Ladson-Billings (1999) emphasized critical race theory and its structural components as the foundation for improving how instructors engage diverse groups of students. When implemented with fidelity, culturally sustaining (Paris, 2012) pedagogical practices can construct a bridge between home and school cultures enabling students to make connections between them instead of viewing each as a completely separate entity. This inherent since of connection enhances school culture and creates since of belonging, particularly true for African-American students who may feel ostracized by their gifted peers.

Scholars suggested that low socioeconomic status, coupled with other factors contributes to limited literacy, and poor academic achievement in schools (Brooks-Gunn \& Duncan, 1997; Haveman \& Wolfe, 1995; Teachman, Paasch, Day \& Carver, 1998; White, 1982), thus it is identified as a prominent contributor to the achievement gap. In his analysis of the 1966 Coleman Report, White (1982) insisted there is a significant relationship between academic achievement and socioeconomic status due lack of educational resources provided at home. With regard to educational attainment and progression, family income is considered to be an influential factor: 
The abilities of parents and their educational choices jointly determine the level of family income and the quantity and quality of both time and goods inputs (or "home investments") that parents devote to their children. Children's ability and the levels of parental income and home investments in time and goods determine the schooling attained by children and, through schooling, the level of postschool investment. (Haveman \& Wolfe, 1995, p. 1,834)

Limited financial resources not only affects availability of resources, but often reflects parental education attainment and may contribute to parents' value for their child's education and involvement therein (Lee \& Bowen, 2006). In spite of poverty and its related hardships that students experience, schools are held accountable for their progress towards narrowing the achievement gap and thus are responsible for educating all students, rich and poor.

Although narrowing over the last few decades (U.S. Department of Education, 2009a, 2009b), substantial disparities in achievement still exist not only for minority students, but also for high poverty students and those with exceptional education needs. These struggling groups, often referred to as the gap-groups, are primarily composed of low socioeconomic students and African-Americans. In an effort to hold schools accountable, particularly those serving low socioeconomic communities and designated as low performing, federal and state officials have adopted NCLB-based incentive programs aimed at increasing achievement for gap groups. One such effort sought to increase minority enrollment in AP courses and reduce the cost of AP examinations for minority students attending urban schools (Kyburg et al., 2007). 


\section{A Place for the Gifted: Advanced Placement}

Following World War II, U.S. education officials observed a downward trend in the number of high school graduates pursuing higher education, indicating the immediate need of a sustainable solution. In response, the Ford Foundation generously allocated grant funding to support research investigations to find tentative solutions for improving educational outcomes (The College Board, 2003). The Foundation's primary goal was to enhance student matriculation through colleges and universities and the resulting study recommended the creation of a curriculum at the secondary level, which armed students with critical skills necessary to succeed in college. With these considerations in mind, higher education officials from several Ivy League universities collaborated to develop high school coursework that aligned with college curriculum, challenged student academic abilities and nurtured critical skills. Thus, throughout the 1950s high schools across the United States initiated Advanced Placement (AP) courses. Within the first five years of inception, 1,229 seniors enrolled in AP courses and 130 colleges accepted them as college credit (Kyburg et al., 2007). In the decades that followed, coursework expanded to all secondary grade levels. The number and type of courses available for college credit through AP programs flourished.

It was not until almost 30 years after its creation that The College Board encouraged its participating schools to make a concerted effort to reach out to low SES and minority students in AP courses (The College Board, 2003). In 1988, the Jacob K. Javits Gifted and Talented Students Education Act subsidized state departments of education and local school districts in their efforts to develop research strategies to support the needs of nontraditional (e.g., special education, gifted and talented) learners 
(Ford, 1998; Gubbins et al., 2014; Renzulli, Callahan \& Gubbins, 2014; Stephens \& Karnes; 2000; U.S. Department of Education, 2015). The most profound section of the Javits Act highlights the need for education officials to give priority to the learning needs of students who are of low socioeconomic status, limited English proficiency or disabled (U.S. Department of Education, 2015). Despite the Javits Act's conspicuous demand for prioritizing underrepresented students in gifted programs, many states continued to identify eligible students using an archaic, culturally-null federal definition established in 1978. In response to a persistent need for inclusiveness in gifted classrooms--coupled with the rapidly changing population demographics - the U.S. Department of Education (Office of Educational Research and Improvement) revised its definition for the identification of giftedness in 1993:

Children and youth with outstanding talent perform or show the potential for performing at remarkably high levels of accomplishment when compared with others of their age, experience, or environment. These children and youth exhibit high performance capability in intellectual, creative, and/or artistic areas, possess an unusual leadership capacity, or excel in specific academic fields. They require services or activities not ordinarily provided by the schools. Outstanding talents are present in children and youth from all cultural groups, across all economic strata, and in all areas of human endeavor. (p. 26)

The Javits Act, aligned with the new definition of giftedness and state modified versions that followed, were widely accepted as viable for identifying giftedness in students of color and have since been used to develop alternative options for recruiting diverse 
groups of students into gifted classrooms. One such recruiting option that developed is "open enrollment".

In recent years, AP courses have transformed options for academic achievement at the secondary level. Moreover, their rigorous course work and culminating examinations are designed to be prominent predictors of college success (Adelman, 1999; Dougherty et al., 2006; Klopfenstein, 2004; Solorzano \& Ornelas, 2004; Zarate \& Pachon, 2006). The expansion of AP courses in high schools across the country has led to federal and state government initiatives, as well as private investors (e.g., Bill and Melinda Gates Foundation Early College Initiative) to promote rigorous, collegepreparatory classes such as Advance Placement as "a model of rigorous curriculum for high school learners" (Kyburg et al., 2007, p.181). In turn, this encouraged more high schools to adopt AP curriculum in multiple content areas to enhance perceptions of school quality.

In response to federal financial support, colleges and universities have transcended high school grade point averages and college entrance exams (American College Test, Scholastic Aptitude Test) as primary criteria for admission and instead are considering AP coursework and examination scores as alternatives. High school students receiving AP exam score between 3 and 5 are considered exemplary and innately equipped to achieve at higher academic levels and may be considered for possible exemption from primary undergraduate coursework (Kyburg et al., 2007; Lichten, 2007; The College Board, 2003, 2005; The Princeton Review, 2017). Such class exemptions allow prospective college students and their families to mitigate the time and cost of higher education, thus saving money and graduating in earlier. Further many colleges and 
universities give considerable attention to AP courses, as part of their admission criteria and course placement procedures (Speroni, 2011). Scholars note that in comparison to their peers in comprehensive courses, gifted students who score below the required minimum on AP examinations are still better prepared for college and as a result, mitigate excessive expense by graduating within five years (Callahan, 2003; Klopfenstein, 2004; Ndura et al., 2003; Olszewski-Kubilius, Lee, Ngoi \& Ngoi, 2004; Speroni 2011; The Princeton Review, 2017) . The post-secondary benefits of AP programs are substantial, yet African-American students remained disproportionately under-represented in gifted programs. Education officials recognized this disparity and their reform efforts shifted towards increasing opportunities for disadvantaged students and increasing diversity in gifted classrooms.

With the federal government acknowledging a revised definition of giftedness, minority participation in Advance Placement classes experienced a dramatic increase between 1998 and 2002, as some high schools across the country reported as much as $77 \%$ of their minority students were enrolled in AP courses (Dougherty et al., 2006; Kyburg et al., 2007). Yet, many urban high schools, the majority of which serve large populations of low socioeconomic and ethnically diverse students, did not offer Advance Placement courses. Therefore, in an effort to expand AP course adoption among high schools, the Secretary of Education established NCLB policies to allocate grant funds to school whose efforts to expand their programs lead to increased pass rates and exam scores (Kyburg et al., 2007). In spite of federal and state government support and financial incentives, scholars documented disproportionately fewer minority students participating in AP courses at the secondary level (Dougherty et al., 2006; Ford, 1998; 
Ford \& Grantham, 2003; Ford \& Thomas, 1997; Fordham \& Ogbu, 1986; Grantham \& Ford, 2003: Kao \& Thompson, 2003; Klopfenstein, 2004; Oakes, 1990; Solorzano \& Ornelas, 2004; Van Tassel-Baska, Feng, Swanson, Quek \& Chandler, 2007; Van Viadero \& Johnston, 2001). Edwards and Duggan (2012) noted that among high students enrolled in Advance Placement in the 2011-2012 academic years, 57\% of students are White, $10.3 \%$ are Asians 10.3\%, 9\% are African-American, and 17\% are Latino/a. This shift in minority enrollment is surprising, given that data from the previous school year shows African-Americans comprise 13\% of the total number of AP students, (U.S. Department of Education, 2010). Even fewer African-American students are completing AP courses and passing subsequent examinations to receive college credit (The College Board, 2006; U.S. Department of Education, 2010). Although AP examination scores are lower for minorities, educators and researchers alike insist that participation in AP coursework provides the rigorous learning environment necessary to equip them with the critical skills used as indicators of future college success (Dougherty et al., 2006; Gubbins et al., 2014).

In the case of urban schools, achievement data for minority populations are critically important measures of accountability. Academic data for those students from impoverished communities is vital, due to their limited access to academic resources and expanded learning opportunities (Kao \& Thompson, 2003; Klopfenstein, 2004). Analyses of inequities surrounding minority access to Advance Placement courses, suggest schools must enact curriculum changes and implement strategic multicultural resources to effectively increase and sustain minority enrollment in gifted courses (Ndura et al., 2003; Oakes, 1990; Zarate \& Pachon, 2006). Therefore, the use of AP courses to narrow the 
achievement gap would require a significant increase in minority participation, particularly in urban schools where underrepresented populations are prevalent. Accomplishing this objective necessitates moving beyond current studies in deficit thinking and engaging in insightful, qualitative exploration of gifted students to better understand the contextual nuances of their academic experiences and determine the extent of their needs.

Historically, Advance Placement courses were comprised of a homogeneous group of students, predominately White males, with a few females and Asians representing diversity (Ford, 1998). Students were identified as gifted or talented following successful performance on standardized assessments such as Scholastic Aptitude Test (SAT) (Chenoweth, 1998; Klopfenstein, 2004) or Intelligent Quotient (IQ) exams coupled with district defined abilities for determining giftedness (Ford, 1998; Ford \& Thomas, 1997). Despite their best efforts, African-American students were more often than not, excluded from participating in school-based gifted programs (Ford et al., 2001: Ford \& Grantham, 2003; Siegle, 2001). However, with the adoption of No Child Left Behind and subsequent policies to diminish the achievement gap, gifted classrooms became increasingly more diverse. In their presentation to stakeholders on defining equity in the context of AP courses, Edward and Duggan (2012) insists that educators "eliminate barriers that restrict access to AP for students from ethnic, racial and socioeconomic groups that have been traditionally unserved" (p. 8). Not alone in his sentiments, many of Duggan's predecessors recognized the under-representation of minority students in gifted courses (Baldwin, 2004; Edward \& Duggan, 2012; Ford \& Grantham, 2003; Fordham \& Ogbu, 1986; Fordham \& Thomas, 1997; Grantham \& Ford, 
2003; Klopfenstein, 2004; Ndura, Robinson \& Ochs, 2003; Solozano, 2004). Scholars offered a variety of solutions, including performance-based assessments (Van TasselBaska et al., 2007) and observations of varied tasks (Passow \& Frasier, 1996) neither of which proved viable for the long-term.

Several researchers suggested the use of alternative assessment measures to identify gifted minorities (Ford, 1998; Frasier, Garcia \& Passow, 1995), including "culturally sensitive instruments, multidimensional strategies, and broader philosophies" (Ford, Harris, Tyson \& Trotman, 2001, p. 56). As the governors of Advanced Placement, The College Board recognized its responsibility to spearhead an initiative to combat traditional notions of minority achievement and in 1990, in conjunction with numerous cooperate sponsors, launched its national EQUITY 2000 program. The program sought to increase participation in AP courses for disadvantaged (e.g., low socioeconomic, minority) students using a systemic approach, uniting schools, districts, states and postsecondary institutions to narrow the gap in college acceptance rates between marginalized groups of students and their White peers (The College Board, 2001). At the school level this meant increasing access to college preparatory coursework through deliberate dismissal of academic tracking for admission in to AP courses, as well as providing professional development for instructors, afterschool academic and assessment preparatory programs for students and academic support training for parents (The College Board, 2001). In its summative analysis of the EQUITY 2000 program participation across thirteen states, The College Board (2001), noted promising improvement in the number of minority students enrolled in AP courses, particularly in mathematics and science. 
In their ongoing efforts to mitigate the deficit-model of identification, many high schools are shifting their AP enrollment process from traditional measures (grade point averages, standardized test scores) to open enrollment format, in which students self-elect to participate. Although not yet widely accepted one such program, AVID (Advancement Via Individual Determination), began in 1980 by a veteran classroom teacher and is based on the philosophy that students held to high academic standards will succeed with the necessary academic and social supports (AVID, 2017). AVID incorporates "researchbased strategies and curriculum" (AVID, 2017) and educator training as a part of a K-12 program designed to target marginalized students in the "academic middle" and help them acquire the critical skills needed to enter post-secondary institutions. Group of students are selected as candidates and upon review of their academic history are selected to participate based on their overall latent ability to be successful in rigorous classes and matriculate into college. The AVID program emphasizes sociocultural supports, including positive teacher-student and student-student relationships. Data from the 201314 school year indicate substantial growth from its modest classroom beginnings, with more than 4,800 schools actively participating in the program. Data on AVID senior high school students shows: $74 \%$ take part in Free-or-Reduced Lunch, $72 \%$ identify as gapgroup learners (i.e., African-American) and 99\% of seniors graduate high school within four years (AVID, 2017).

Although national programs like the examples described above are promising, the issue of under-representation among ethnic minorities remains a concern among researchers and practitioners alike. Many states and districts continue to struggle with finding a viable solution to the problem. These efforts are more feasible given the 
provision of the recently enacted, Every Student Succeeds Act (ESSA) bill, which suggests autonomy for state departments of education and schools districts in "develop[ing] their own strong systems for school improvement based upon evidence, rather than imposing cookie-cutter federal solutions like the No Child Left Behind Act did" (The White House, 2015). However prior to ESSA revisions some states had already begun their journey to school improvement by taking matters into their own hands.

In a statewide effort to decrease a substantial gap in achievement between African-American and White students, in 2008 the Department of Education within the state site of this study, formulated a program called AdvanceState (pseudonym). AdvanceState is a statewide initiative designed to recruit diverse students into Advanced Placement classes. Despite its humble beginnings as targeted math and science initiative, to date more than 100 high schools across 74 districts have adopted this initiative and more than 4500 Advanced Placement and Advance Program (Pre-AP) attended contentbased training sessions. The primary goal of AdvanceState is to increase accessibility to post-secondary education for students of color, by arming them with the critical thinking skills needed to prepare them for rigorous coursework. To achieve the goal of increased representation for marginalized groups, schools participating in AdvanceState forego traditional measures for admitting students into gifted courses and instead use an open enrollment protocol to recruit students into rigorous classes. Moreover, counselors, teachers and administrators are able to recommend students to participate in AP classes. Upon consent, these students transition into one or more classes during the next available enrollment period (i.e., beginning of a semester, trimester or school year). In a state issued report, the State Commissioner of Education, commended AdvanceState saying: 
Clearly this initiative is having a positive impact...AdvanceState students have repeatedly demonstrated that in an environment of equity, inclusion, and high expectations, not only will they enroll in rigorous coursework, but also they can succeed and gain a competitive edge over other students.

In addition to support from AdvanceState, students in this and 12 other states, receive subsidized cost for AP examinations. This type of open enrollment initiative is not unique to this state and although response rates have been modest, other states have adopted protocols, which allow students to self-nominate into the gifted courses (Coleman, Gallagher \& Foster, 1994).

To demonstrate the continued need for its existence, state administrative data indicate that AdvanceState conducts longitudinal studies to evaluate growth for those students enrolled in AP classes at participating schools in comparison to nonparticipating schools. Study results are measured by comparing qualifying student scores (a score of3 or higher) on culminating AP examinations among the two groups of schools. Further, additional research indicates that American College Test (ACT) scores and college enrollment rates are significantly higher for AdvanceState participants than students not enrolled in the program. The AdvanceState open enrollment initiative served as the contextual backdrop providing a distinct environment for the exploration of interactions among both gifted and traditional peers.

\section{Peer Group Experiences in Academic Contexts}

Throughout the literature, researchers identify various social elements as factors that contribute to lags in student achievement. Influential social elements such as culture, family, peer groups, audiovisual media, social media and teacher perception inhibit or 
contribute to student achievement. In their assessment of causes for the achievement gap, scholars assert that adolescents are most influenced by their peers' perception of them, as they have a stake in maintaining these social relationships (Nelson \& Debacker, 2008; Robertson \& Symons, 2003; Ryan, 2000; Steinberg, Brown \& Dornbusch, 1997). Peer relationships are a vital component of student beliefs, academic self-confidence, subsequent academic achievement (Ryan, 2000; Wentzel, 1991) and adolescents often hold the opinion of peers at greater value than that of their parents (Burke \& Sass, 2013; Darensbourg \& Blake, 2014; Steinberg et al., 1997). Within the existing research literature there are some studies that examine social and cultural factors related to peer influence (Chen, French \& Schneider, 2006; Ford, 1998; Hanushek, Kain, Markman \& Rivkin., 2003). This section summarizes key findings from literature on the relationship between peer groups and achievement, in particular minority peer interactions as a component of academic success.

Ryan (2000) identifies schools as social entities for adolescents where interactions among peers are prevalent and seemingly influence decisions regarding behavior and achievement. She contends that an in-depth discovery of peer group contexts is needed to understand the vital role schools play in facilitating peer relationships and manifesting their influential behavior. Ryan refers to an emerging peer group as one in which members choose to have more frequent interactions with beyond the classroom setting. At the high school level, students spend significantly more time engaging in shared interactions with their peers through conversations, behavior and experiences (Schunk \& Pajares, 2001; Zimmerman, 2003). These interactions occur amongst various peer subgroups in both academic and nonacademic contexts. Moreover, the context of these 
interactions play an important role in determining the impact of peer relationships on adolescent decision-making processes (Ryan, 2000).

Adolescent derived decisions from opinions, recommendations and shared experiences of peers, thus high school students often seek peer approval in order to finalize both social and academic decisions. Peer pressure is a social construct defined within various contexts pertaining to adolescent development, behavior and academic success. Despite its ambiguity, experts insist that peer pressure deserves significant attention due to its prevalent influence on adolescent attitudes and academic achievement (Ryan, 2000; Viadero \& Johnston, 2001; Zimmerman, 2003), which peaks during high school years. Peer pressure resulting from school social groups affects both positive and negative intrinsic views on achievement and encompasses a realistic context for students (Ryan, 2000; Schunk \& Pajares, 2001; Zimmerman, 2003). Because of this pressure, individual beliefs that members deem as negative are less likely to reappear, while those upheld as positive are more likely resurface. It is this social phenomenon that underlies Roberston and Symons' (2003) argument that adolescent self-perception of educational capabilities are a function of peer group influences.

Peer relationships are a primary factor in shaping adolescent aspirations for academic success. Perhaps unknowingly, peer group members participate in what Davies and Kandel (1982) refer to as a reciprocal system of influence in which interpersonal discussions among peers, impacts decisions about educational ambitions. Davies and Kandel argue that although a complementary process exists, the resulting influences are weak predictors of educational attainment when compared to other factors such as parental involvement and socioeconomic status. Later research confronts this idea 
insisting that friends are academically similar (Nelson \& Debacker, 2008; Ryan, 2000), and that peer relationships explicitly alter academic self-efficacy in teenagers (Schunk \& Pajares, 2001). For high school students, increased time spent interacting with peers and the inherent desire to assimilate into subgroups creates interpersonal experiences that may provide motivation to academically improve or instead stimulate apathy for learning.

Throughout literature, the effect of peer influence on minority student achievement is broadly explored through a psychosocial, developmental lens. The primary focus of these studies was to uncover cultural assumptions underlying the achievement gap and analyze the resulting academic deficiencies for minority students. Fordham and Ogbu (1985) explored the academic experiences of African-American students in the context of predominately White schools. After reviewing data from a preliminary ethnographic study, Fordham and Ogbu (1985) contend that educational challenges observed in African-American students are attributed to their "inordinate ambivalence and affective dissonance" with regard to the expectation of academic performance (p. 2). She references earlier assertions by her future co-author, John Ogbu, who emphasized a theory of resistance and a culture of apathy toward academic pressures resulting from the historical oppressive experiences of Black Americans. Fordham acknowledges Ogbu's assertions as relevant; however she contests their exclusivity by highlighting verbal accounts of minority students who were successfully matriculating through Advance Placement courses in predominately White high schools — despite experiencing oppressive ridicule from both sides.

Scholars (Baldwin, 2004; Ford et al., 2001; Klopfenstein, 2004; Kyburg et al., 2007; Viadero \& Johnston, 2001) have referenced both Fordham and Ogbu in their 
studies on academic achievement in underserved populations, citing a distinct connection between social pressures and classroom performance. In her analysis of existing research, Baldwin (2004) finds that culture dictates the attitudes in minority communities, which then transcend into peer groups resulting pressure on individuals to conform. Further, peer relationships may serve to amplify existing doubts about minority student's perception of their own academic ability. This may help to explain lags in achievement for students of color, as well as their adverse rates of enrollment in Advance Placement courses.

In order to increase minority participation in AP classes, many high schools have removed selection protocols, allowing any interested student to enroll voluntarily. Following these open enrollment initiatives, analyses reveal that many qualified students still choose not to enroll (Viadero \& Johnston, 2001). The insightful rhetoric of one African-American high school student in response to low enrollment numbers at her school captures the essence of the problem - "A lot of times, I think [we] are discouraged from being in Honors or AP classes because they see no one else [like them] in those classes" (Viadero \& Johnston, 2001, p. 3). This discouraging perspective aligns theorists who maintain that peer groups influence individual responses to changes within school environments, which may adversely affect self-perceptions of academic ability (Berndt \& Keefe, 1995; Pajares, 1996; Ryan 2000). These findings suggest that cultural norms and peer influence persuade gifted minority students to neglect their intellectual abilities and deny opportunities to challenge their skills in advance courses (Hibert, 2000; Reis, Hibert, Diaz, Maxfield \& Ratley, 1995). Research on peer relationships studies are predominately quantitative analyses (Berndt \& Keefe, 1995, 2002; Nelson \& Debacker, 
2008; Zimmerman, 2003) demonstrating the need for qualitative exploration into the socio-academic experiences of gifted minorities and how they perceive their peers as influencing them. Despite open enrollment efforts at some schools, it is currently unknown to what extent peer groups and subsequent relationships actually influence the academic experiences of minority students, particularly those in gifted programs.

\section{Self-Efficacy Theory: Perception of Academic Ability}

Albert Bandura introduced the concept of elf-efficacy during the 1970s and 1980s; a concept rooted in social cognitive theory. He notes that self-efficacy and human achievement are manifested through cognitive developmental processes such as motivation and selection, as well as behavioral interactions (Bandura, 1982; 1986; 1993). Self-efficacy profiles an individual's belief in his or her own ability to coordinate and complete a specific task, in order to achieve an expected outcome. Bandura identified several internal factors: motivational, cognitive and selection, through which self-efficacy can shape individuals' beliefs about their own ability to accomplish a task. Each of these levels, in turn, go on to influence achievement factors such as, aspirations, enthusiasm and academic success (Bandura, 1993). In other words, in order to appraise one's own capabilities effectively, an individual must engage in several intrinsic processes, which will ultimately determine their desire, motivation and commitment to success. Moreover, researchers note that self-efficacy is a prominent factor contributing to the range and value of human meaning (Bandura, 1986; Pajares, 1996).

Bandura (1986) noted that one's ability to surmount a difficult task required them to regulate thought, emotion and action. In addition, individuals must demonstrate a strong self-belief that they possess required skills-set to complete the task successfully In 
his analysis of self-efficacy, Pajares (1996) acknowledges Bandura's (1986) inclusion of cognitive and emotional influences on self-efficacy, yet he continues that social interactions and psychological well-being also contribute to an individual's efficacious strength. Both authors agree that personal confidence is a viable predictor of accomplishment (Bandura, 1986; Pajares, 1996): “...outcomes people expect are largely dependent on their judgments of what they can accomplish, outcome expectations are unlikely to make much of an independent contribution to predictions of behavior when self-efficacy perceptions are controlled" (Bandura, 1986 p.241). Thus despite its individualized function, self-efficacy is clearly a multidimensional process influenced by a both intrinsic and extrinsic factors.

Self-efficacy is identified as having a significant effect on student academic achievement at all grade levels (e.g., Kindergarten through 12 ${ }^{\text {th }}$ ) (Bong \& Skaalvik, 2003; Pajares, 1996, 2003; Schunk, 1991, 1995; Schunk \& Pajares, 2001). In the classroom, efficacy determines a student's ability to gauge their inherent capability to complete a task or reach a goal and is vital in helping to shape their academic success. Bandura (1993) contends self-efficacy is an essential component of cognitive development and as such, it directly influences a child's belief in their ability to master challenging curriculum, thus influencing their motivation and achievement. Sources of self-efficacy are believed to differ with regard to ability, gender and race/ethnicity (Usher \& Pajares, 2006). Moreover, high-ability learners are efficacious, ambitious and driven to succeed despite unexpected setbacks (Bandura, 1993), while those with low self-efficacy tend to view challenges as overwhelming and stressful. For gifted students, self-efficacy may influence the confidence to successfully complete rigorous coursework. 
For adolescents, high school presents a challenging adjustment period in which their academic work will continuously align with that of their peers, creating a competitive nuance with negotiable effects. Navigating this incessant, comparative process may affect students' appraisal of their own capabilities, however those who view themselves as efficacious are successful in managing their own learning. In this regard, students inherently challenge themselves to recover from failed tasks by analyzing and correcting errors, all while gaining satisfaction from the experience. Self-efficacy exerts a multidimensional impact within the classroom including acting as viable predictor of academic performance (Bong \& Skaalvik, 2003; Pajares, 1996, 2003; Schunk, 1991, 1995; Schunk \& Pajares, 2001), behavior (Bandura, 1993), and cognitive ability (Bandura 1986, 1993, 1997; Schunk, 1991).

A student's natural drive to assess their own abilities and set goals based on their strengths, results in rigid commitments and increased effort toward achievement. Pajares $(1996,2003)$ notes that this appraisal process among students is further complicated by variables directly affecting academic performance such as practicality, prior experience and achievement, natural talent, demographics, socioeconomic status and mental health. In addition, those students who lack task-specific confidence, struggle to visualize success, inundate themselves with self-doubt and often forecast their own demise, thus making achievement even more difficult (Bandura, 1993; Pajares, 1996). Academic and personal accomplishments require positive self-belief, intrinsic enthusiasm and ability overcome disappointment. However, Bandura (1993) argues that when pronounced, selfefficacy can define aspirations, align effort, and even predict academic achievement. Researchers cite high-achieving students whose efficacious behavior, despite the 
challenge level, provides them with an elevated sense of self-guidance, inherent motivational support, which for them prefaces a successful outcome (Bandura, 1993; Bong \& Skaalvik, 2003; McCoach \& Siegle, 2003; Pajares, 1996; Schunk \& Pajares, 2001). Efficacious students tend to recover quickly from setbacks with minimal downtime, display resilience and exert positive work ethic. In contrast, students with low self-belief, particularly those who experience failure, are likely to avoid challenging tasks, have weakened persistence (Bandura, 1993; Ford, 1998) show decreased interest and effort (Bandura, 1997; Schunk, 1995; Schunk \& Swartz, 1993). As a result, these students often have lower academic grades and assessment results, and experience psychological effects such as academic anxiety and depression.

\section{Student Identities}

Adolescence marks a period of physical, emotional and social change "during which individuals are continually structuring and shaping their sense of self" (Chavous, Hilkene, Schmeelk-Cone, Caldwell, Kohn-Wood \& Zimmerman, 2003, p. 1,076). For teenagers developing a secure identity is a multifaceted process filled with contextual complexities influenced by environmental, social, racial, cultural, gender and academic factors. Throughout this crucial period of development students may find themselves struggling to find their place (or not) in one or more social groups. Researchers often connect identity exploration to peer group relationships (Steele, Spencer \& Aronson, 2002; Tajfel \& Turner, 1986), voluntary activities (Barber, Eccles, Stone, 2001), and to both positive and risky adolescent behaviors. Tajfel and Turner's (1986) social identity theory suggests that individuals develop an identity by placing themselves into categories based on their social relationships with others- others that have also been grouped into 
categories based on predetermined characteristics (Ashford \& Mael, 1989). Within organizations including educational institutions, social relationships have a complex role in identity development as each person struggles to define themselves in relationship to the group (organization), subgroups and as individuals (Ashforth \& Mael, 1989). For gifted students, this process may be intensified in their attempt to solidify an identity that will be accepted among both gifted and traditional peers.

\section{Racial Identity}

When compared to their White peers, African-Americans students experience greater struggles in adhering to cultural norms while attempting to establish individual identities. For African-American students, the extant literature identifies racial identity as one of many factors contributing to lags in academic achievement for this subgroup (Chavous et al., 2003; Cokley, McClain, Jones \& Johnson, 2011; Ford, Harris \& Schuerger, 1993; Ford-Harris, Schuerger \& Harris, 1991; Fordham, 1988; Fordham \& Ogbu, 1986). The conflicting perspectives on the significance of education for minority and dominant cultures exacerbates the process of identity development for gifted students of color by. For African-Americans, the idea of race centrality, connectedness to one's race, as well as their beliefs and values associated with culture, determines the level of significance a person and his or her judgement has to their lives (Rodgers, 2008). Students possessing higher levels of race centrality have increased sensitivity to the opinions of their peers and tend to struggle more with identity development, whereas those with lower race centrality are less affected by such judgments.

Elder generations of African-Americans held steadfast to the idea that education is a characteristic of the dominant culture (i.e., Whites) and to pursue it shows minimal 
appreciation for one's own culture (Ford et al., 1993; Fordham, 1988; Ford-Harris et al., 1991). In contrast, as younger generations of African-Americans are entering institutions of higher education at much higher rates (U.S. Department of Education, 2016), they clearly possess a more progressive attitude toward educational attainment, acknowledging the benefits of social and occupational mobility and perhaps signaling the revitalization of this once cultural norm. Despite their efforts, there remains a negative stigma associated with African-American children particularly males with regard to academic achievement and social behavior. Scholars argue this affirms a deficit mindset among educators who are then less likely recommend high achieving minorities for gifted courses, (Cokley et al., 2011; Ford et al., 1993; Ford \& Harris, 1997; Fordham \& Ogbu, 1986) thus contributing to the trend of under-representation.in gifted programs. Ford and Harris (1997) contend that these embedded social constructs coupled with cultural expectations shape how African-American students view themselves and their position within the racial group-- a sentiment they refer to as racial identity.

Minority students' adverse self-perceptions resulting from societal bias are significant to identity development and may affect beliefs about academic achievement (Chavous et al., 2003; Ford \& Harris 1997: Graham \& Anderson, 2008). For academically gifted African-American students these beliefs may lead to anxious feelings about whether to identify with more with their racial or their academic identities. The literature discusses the impact of racial identity on several factors, yet among scholars agree on the presence of a "dual-identity" (Rodgers, 2008, p. 115) that the academically gifted must contend as they explore individuality (Chavous et al., 2003; Cokley et al., 2011; Graham \& Anderson, 2008; Tang, McLoyd, Hallman, 2016). Thus for these 
students the level of connectedness with their racial group will either provide the motivation to achieve or embrace negative stereotypes and "group identification, then, either exacerbates or buffers discrimination effects" (Chavous et al., 2003, 1078). Buffering allows positive racial identity values and connectedness to the group to permeate while creating a shift in academic self-concept (e.g., efficacy) and achievement. The socioemotional experiences associated with racial identity for gifted AfricanAmerican students vary greatly and many struggle when forced to negotiate a dualidentity while trying to establish who they will become.

\section{Academic Identity}

The ability to define oneself as a student is a complex concept frequently influenced by social, cultural and developmental dynamics that may intersect each other within one individual. The task of identity formation as it relates to academic contexts is further complicated a student's motivation (or lack thereof) to succeed in school and is referred to as "academic identity." Within educational research, academic identity is a multidimensional concept investigated by researchers within a variety of contexts, including adolescence, higher education, race and ethnicity. In their analysis of motivational and contextual factors affecting identity formation in middle school students, Roeser and Lau (2002) state:

The challenge facing researchers of adolescence today is that of studying the complex process of identity formation and development during adolescence in particular activity settings such as the home, the school and afterschool settings. Such 'situated studies' can advance our scientific understanding of adolescent development and inform educational and mental health policy and practice. ( $\mathrm{p}$. 
92)

For minority students, establishing an academic identity may be an arduous task viewed as an "either-or" option, instead of a multifaceted concept of self, encompassing social, cultural, psychological, cognitive and developmental dynamics that intersect and manifest themselves into distinct individual forms.

As previously discussed, African-American students hold a double-edged identity sword as they battle cultural and academic identity components that frequently contribute to socioemotional issues. The effects of this identity conflict may be detrimental for some students wherein their academic motivation is completely deterred causing a decline in achievement. Cokley and his colleagues (2011) refer to this phenomenon as academic disidentification; wherein a student becomes increasingly disconnected from his or her academic efficacy, which in turn negatively affects the motivation to perform well in school. Academic disidentification affects all students, but is of particular concern for African-American students whose lags in achievement are reflected in the persistent achievement gap found in large-scale assessment results (e.g., NAEP). Moreover, such concerns are amplified for gifted minorities as their identities and inherent commitment to their racial group are often called into question because dedication to achievement demonstrates value for the ideals of the dominant culture. Graham and Anderson (2008) insist that such social barriers continually force gifted students of color to choose between their racial identity in an effort to confirm their group affiliation or pursuit of educational attainment as a means of mobility.

In her effort to uncover the nuances of academic identity among Puerto Rican students, Flores-Gonzalez (1999), highlights diverging cultural perspectives between 
African-American students as the underlying premise for their distinct perspectives on academic success. She notes that Puerto Rican students view academic achievement as a middle class measure of success that is attainable without the overwhelming risk of feeling ostracized by their peers for acting White, thereby having minimal influence on how they describe their academic identities (Flores-Gonzalez, 1999). In contrast, several prominent studies argue that for African-American students, cultural acceptance is vital component of identity creation. Feelings of discontent arise from what Smith (1989) argues is the inability of individuals from a particular race to define themselves separately from their racial membership. Thus African-American students often find themselves at a crossroads between achievement and acceptance (Ford, 1998; Ford et al., 2001; Ford \& Thomas, 1997; Grantham \& Ford, 2003), finding it difficult to solidify who they are in academic contexts for fear of racial group alienation. According to researchers, this may partially explain why many gifted African-American students "camouflage their [academic] abilities to be accepted socially by their peers" (Fordham \& Ogbu, 1986; Grantham \& Ford, 2003, p.21).

For educators, the limited understanding and training on cultural issues requires additional insight to create intentional strategies target the complex needs of this unique group of students. To understand the diverse needs of students of color, educator may choose seek information from the most reputable source — the students themselves.

\section{Student Voice}

The concept of student voice emerged as an important component of modern education research; as many institutions struggle to implement reform efforts aimed at improving student achievement effectively. Not an entirely new concept, student 
discourse was initiated during the 1960's and 1970's as students demanded the right to decision-making power within the school and has recently resurfaced with the insurgence of educational reform efforts aimed changing academic outcomes for students. Various researchers have used participant voice across genres such as education, politics and healthcare. In her foundational analysis on student voice. Orner (1992) asserts that it is the responsibility of education researchers to ensure student voice remains authentic through their careful consideration when selecting the language, context, subjectivity and examination lens. Mitra (2004) notes that in wake of trying to improve student academics, schools neglect to seek assistance from the source of the problem-the students themselves.

Following the mid-twentieth century the resurgence of student voice within education as a tool for initiating change in schools became a valuable component of improving pedagogy, curriculum and student-adult relationships because they have unique perspectives that cannot be accurately portrayed by adults (Fielding, 2004b; Fielding \& Rudduck, 2004; Mitra, 2004; Mitra et al., 2012). The use of student voice as a tool for change is met with a power struggle stemming from the adults versus students systemic hierarchy (Mitra et al., 2012). This presents the challenge of holding adults accountable for their interactions with students to ensure students are actively heard and not overshadowed by adult status. Adult reenactment of student voice minimizes its value; therefore, it requires that adults encourage and actively engage students to express themselves freely which in turn enhances a sense of belonging and acceptance. At the very least student voice includes sharing opinions on school-level issues, however, adults should also understand that student voice can encompass a variety of activities that 
"encourage reflection, discussion, dialogue and action on matters that primarily concern students" (Fielding, 2004a, p. 199) and may in turn benefit the communities they live in (Fielding, 2004a; Mitra 2012). Regardless of the activity, positive adult interactions throughout schooling is essential to the student voice dynamic and it is the responsibility of adults to create positive encounters to ensure the process is successful (Mitra et al., 2012). Student voice is powerful and can act as a "catalyst for change in schools" (Mitra, 2004, p. 652). At the classroom level, students afforded opportunities to express themselves improve academically and when they collaborate with teachers may help to increase effectiveness of curriculum and pedagogical practices (Flutter \& Rudduck, 2004; Oldfather, 1995; Oldfather \& Thomas, 1998; Rudduck \& Flutter, 2000).

Scholars report that students do not regularly communicate with school adults because they are made to feel as if their opinions do not matter (Mitra, 2004) and that they are powerless to initiate change (Fielding, 2004b; Fielding \& Rudduck, 2002; Mitra, 2004). Educators could learn a lot from students if they would simply listen to them.

Fielding and Rudduck (2002) highlight the importance of integrity and open communication as a part of student voice:

There are many silent or silenced voices - students who would like to say things about teaching and learning but who don't feel able to without a framework that legitimates comment and provides reassurance that teachers will welcome their comments and not retaliate. (p. 13)

The sense of powerlessness and invisibility that students feel due to can lead to disengagement and low self-efficacy, which may contribute to poor academic performance (Mitra, 2004). If educational reforms are truly aimed at improving academic 
performance, then every effort should be made to hear from those who represent the primary variables by which this change is measured - the students.

In her analysis student voice in the classroom through a feminist poststructuralist lens, Orner (1992) insists that context is the underlying component of silenced voices, as students struggle to find instances they perceive as appropriate to voice their opinions. The authoritative hierarchy that exits in schools (e.g., administrator, teacher, and student) places students at bottom and as a result adults may misinterpret student silence as defiance or resistance (Orner, 1992). Within this hierarchy, students often struggle to find, use and maintain their individual power, including their right to use or silence their voice as a tool for change. Student voice and silence stemming from power relations in school are complex and include various internal and external factors that influence the decision to speak or not (Orner, 1992; Mitra, 2004). Student silence is be attributed frequently to factors that adults are unaware of, and in some cases, their silence may be the loudest voice they have to express feelings of injustice in school. Orner (1992) challenges opponents of student voice in education saying:

How power relations in the classroom are manifest is crucial. How do the subject positions inhabited by one student connect with the subject positions of everyone else in the room? How do these multiple identities and positions inform who speaks and who listens? Who is comfortable in the room and who is not? Who was insulted and who did the insulting in the hall just before class? It seems impossibly naïve to think that there can be anything like a genuine sharing of voices in the classroom. (p. 81) 
Within education, tiered levels of power will always exist, however those at higher levels are responsible for cultivating an environment that acknowledges student voice as a valuable component for effective change.

\section{Summary}

Researchers contend both academic and nonacademic factors contribute to the lack of minority enrollment in gifted and talented courses (e.g., Advance Placement). Studies suggest environmental elements, such as socioeconomic status, quality of schools, teacher efficacy, racism and parental education attainment each influences participation and achievement in school (Kao \& Thompson, 2003; Klopfenstein, 2004; Kyburg et al., 2007; Solorzano \& Ornelas, 2004). However, among adolescents, the dynamics of peer relationships are thought to play significant roles in social behavior, self-efficacy, perceptions of academic ability and formation of academic identity. In the case of minority students enrolled in Advance Placement (AP) courses, insight into their unique socio-academic experiences assisted educators in providing enhanced resources and support to ensure their success.

The literatures presents contrasting arguments, identifying multiple characteristics (e.g., pedagogy, poverty, assessment bias) that contribute to the achievement gap crisis. These disheartening factors prompted educators to develop a viable solution to address the stagnant gap elicit the need for alternative approaches to the problem. One such reform effort focused on combating the limited representation of students of color in gifted and talented programs. As a part of No Child Left Behind, the federal government allocated substantial provisions for states working to increase minority achievement through participation in AP courses (Kyburg et al., 2007). The reenactment of ESSA 
supports diverse growth within gifted programs by retaining the Javits Act, which includes revised protocol for data collection and the requirement that states and districts use inclusive language when referring to gifted students (National Association for Gifted Children, 2016). Despite increased diversity within gifted programs across many states (The College Board, 2003, 2006, 2009), minority students are not receiving qualifying scores on Advance Placement exams at the same rate as White students because of ineffective, or in some cases, nonexistent support systems to facilitate their success.

Within the literature, researchers have used a deficit model to approach lags in academic achievement for students of color-seemingly placing them at a disadvantage in various academic contexts. This deficit approach is most prominent in studies that attempted to understand the nuances of high achieving African-American students, surrounded by peers who accused them acting White (Ford \& Grantham, 2003; Ford \& Thomas, 1997; Fordham \& Ogbu, 1986; Ogbu, 1991). However, for students of color enrolled in gifted programs, the deficit model seems less applicable given that they have surpassed the status quo and have, or are at least attempting to diminish their own personal achievement gap. In a 2002 article, Ford and her colleagues insist that educators must move beyond deficit-model thinking in their efforts to identify minority students as gifted (Ford et al., 2002). The authors continue that educators must intentionally encourage minority students to participate in gifted programs and work to ensure adequate supports for successful matriculation (Ford et al., 2002). In essence, what better way to determine how best to support students than by listening? The voices of this unique group of students hold significant value to researchers and educators seeking to understand minority students efficiently navigate sociocultural "need for achievement 
and [the need for] affiliation" (Ford \& Thomas, 1997, p.3) and acceptance. Of particular interest are interactions with peer groups, feelings of self-efficacy, and how these encourage or hinder development of positive academic identities. Zimmerman (2003) maintains that quantifying the impact of peer effects on achievement is a challenging task and determining the impact of peer effects through empirical analyses is complicated by external and internal influences (Hanushek et al., 2003). Given these viewpoints, a qualitative exploration that provides the opportunity for gifted minorities to discuss the tones of their academic experiences provides a personal approach to discover inherent characteristics of social and cultural factors.

Throughout the research literature, several studies examined the link between interactions among peer groups and the effect on academic achievement (Berndt \& Keefe, 1991, 1992; Guay, Boivin \& Hodges, 1999; Robertson \& Symons 2003; Ryan, 2000; Schunk \& Hanson, 1985, 1987; Wentzel, 1991). Still others focused on establishing a relationship between academic self-efficacy and achievement (Bandura, 1977, 1982, 1991, 1993, 1997; Bong \& Skaalvik, 2003; Britner \& Pajares, 2006; Pajares, 1996; Walker \& Greene, 2009; Zimmerman, 1990, 2000). Many of these studies elected to use quantitative methodology to examine these proposed relationships, however inherently absent was the voice of the students themselves, in particular students of color. For those minorities participating in gifted programs, how do they manage the numerous social and cultural nuances as they navigate through academic challenges? I have instead elected to design a qualitative study to capture the experiences of gifted minorities. It is my hope that the descriptive data from this study helps to shape best practices for educators, enabling them to meet the needs of gifted minority populations effectively. 
Reform efforts designed to confront the stagnant achievement gap between minority students and their White peers, have resulted in financial incentives, revised policies, program development and increased representation in rigorous courses. However, the debate continues as to the effectiveness of gifted programs in preparing of students of color to enter post-secondary education. Many question the ability of minority students to successfully matriculate through Advance Placement courses and subsequently pass culminating exams to earn college credit. However with more rigorous coursework comes more responsibility—an identity tug-of-war as gifted minority students struggle to maintain their academic success, while enduring the sociocultural and emotional stress of acceptance (Ford \& Grantham, 2003).

Despite efforts to increase enrollment, overall minorities remain less likely to participate and succeed in Advance Placement courses compared to their White peers. Consequently, an increasing number of colleges and universities assign substantial weight to AP course work as a determinant of admission. However, lags in enrollment and positive examination results means that minority students do not have access to the benefits of college preparatory coursework and are ill prepared for post-secondary opportunities. Politicians, educators, and officials offer suggestions and strategies to address achievement disparities for culturally diverse students. Howard (2003) notes that "inexplicably absent from the discourse have been perspectives from the students" (p. 4). My study responded to gaps in the literature regarding sociocultural experiences of gifted African-American students who participated in open enrollment to gain access to AP courses. I sought to collect anecdotal evidence on how these gifted students perceive their peers as shaping their self-efficacy beliefs and academic identity. As the number of 
minority students in gifted courses continues to grow, it is imperative that educators consider the personal experiences and sociocultural dynamics that influence beliefs about their own ability to be academically successful. 


\section{CHAPTER III: METHODOLOGY}

The purpose of my study is to explore the sociocultural academic experiences of gifted African-American students electing to enroll in Advanced Placement courses through open enrollment. I sought to discover connections between students' academic identity and self-efficacy beliefs, as determined by peer group interactions defined by cultural norms and adolescent social nuances. I was guided by two research questions: (1). What are the perceived influences of peers on the development of self-efficacy and academic identity of African-American students enrolled in Advanced Placement courses? (2). How do African-American students in Advance Placement courses perceive their interactions with peers and cultural influences on achievement, as influencing their overall academic experiences? In particular, I was interested how interpersonal relationships within formal and informal peer groups, classroom peers and selfnominating peers shape academic identity and efficacious beliefs. I used a phenomenological case study research design to capture the sociocultural experiences of African-American students enrolled in AP courses within an urban school district. This qualitative method was effective at capturing student voice, highlighting realistic experiences of this unique group of gifted students. The chapter sections that follow describe the study's design, sample population, study location, methods, study limitations, researcher positionality, as well as data collection and analysis procedures. 


\section{Research Design}

Qualitative methodology allows for in depth exploration of issues in a natural setting to capture the details of participant experiences. Qualitative researchers construct an intricate representation of the human experience that increases understanding and provokes thought within the reader (Strauss \& Corbin, 1990). Miles and Huberman (1994) describe qualitative research as a process by which the researcher explores the normal experiences of individuals or groups with the aim of sharing a holistic view of the data. Although qualitative research provides rich detailed data of human experiences, its small sample size and specific detail limit its generalizability. The process requires the researcher to consider participant experiences and then carefully analyze the details, realize themes and make meaning of complex circumstances (Miles, Huberman \& Saldana, 2014). Qualitative data may result in "thick, rich descriptions that are contextualized" (Leech \& Onwuegbuzie, 2007). I selected a qualitative methodology based on a review of extant literature surrounding access for gifted African-American students and achievement, specifically to address gaps related to peer influence on, selfefficacy and academic identity.

There are multiple research designs within qualitative research. The five broad categories of qualitative research are ethnography, grounded theory, case studies, phenomenology, and narrative research (Creswell, 2009). Ethnographic studies involve the study of an individual or group in a natural setting for an extended period. These observations are transformed into narrative research to retell participants' personal stories within a chronological sequence that may incorporate the researcher's view (LeCompte \& Goetz, 1982). Grounded theory research supports the development of a new theoretical 
approach that ventures beyond the constraints of established frameworks and is developed from the participant's perspective (Corbin \& Strauss, 2014). A case study design allows the researcher to conduct in depth explorations of individuals or group of individuals within similar context while phenomenology enhances the focus of study by narrowing it to a specific context. I discuss the components of each framework inherently support the unique scope of this study in detail later in this chapter.

\section{Phenomenology}

I selected phenomenology as one piece of the collaborative methodology guiding my study. Phenomenology is a methodology in which the researcher explores the nature of experiences as told by the participants themselves and emphasizes the delicacy of individual viewpoints and interpretations of those living through a particular circumstance (Lester, 1999). My study aligns with Lester's (1999) description of phenomenology, focusing on three African-American students and their sociocultural experiences in gifted classrooms. Phenomenology is a methodology, as well as philosophical viewpoint and shares qualities with other qualitative frameworks including ethnography and narrative research. Early philosophers interpreted phenomenology as a method for obtaining participant knowledge through the visualization of their own consciousness and their perception of life events (Groenewald, 2004; Moustakas, 1994). However, the goal of phenomenology, as established by Edmond Husserl in the early twentieth century, was to focus solely on the perspective of the participant-wherein the researcher sets aside preconceived ideas and biases to capture the quintessential reality of the participant (Groenewald, 2004; Lester, 1999; Moustakas, 1994; Sokolowski, 2000; Wertz, 2005). The use of student voice as a tool to highlight the reality of participant 
experiences and perspectives on peer influences emphasizes my study's alignment to a phenomenological viewpoint. Moustakas (1994) contends that the phenomenological researcher "engages in disciplined and systematic efforts to set side prejudgments regarding the phenomenon being investigated (p. 22). Thus, the phenomenological researcher becomes inherently open to listening to participant narratives and capturing the surrounding dynamics to provide genuine insight into their experiences. For this study, viewpoints of selected peers and trusted adults were included to enhance details from student-informant interviews. Phenomenology was an ideal method to elicit the experiences of gifted students in this study to uncover the sociocultural dynamics that help to shape their academic identities and beliefs.

In order to portray the lived experiences of individuals in relation to contextual circumstance researchers must capture reality. Summarizing Husserl's description of phenomenology, Wertz (2005) notes:

This return to phenomena as they are lived, in contrast to beginning with scientific preconceptions, is a methodological procedure and does not imply that such knowledge is false; it simply suspends received science, puts it out of play, and makes no use of it for the sake of fresh research access to the matters to be investigated. (p. 168)

Phenomenology focuses on exploring the realities of participants for this is "the only absolute data from where to begin" (Groenewald, 2004, p.4). The term phenomenon, derived from the Greek work phaenesthai, which means "to bring to light, to place in brightness, to show itself in itself" (Heidegger, 1977). An individual's phenomena are their cognitive interpretation of lived experience and helps to create new perspectives and 
knowledge. In his analysis of phenomenological research, Moustakas (1994) notes that "phenomena are the building blocks of human science and the basis for all knowledge" (p. 26). Lester (1999) continues defining phenomenological research as the discovery of related events as interpreted by persons directly involved with the actual event. In this way, their relation to an identified event seemingly connects study participants. In my study, student interconnections are by designation to a racial subgroup (i.e., AfricanAmerican) and by their voluntary participation in academically gifted programs (i.e., Advanced Placement). Despite the phenomenological connection-- although it is likely that the individual interpretations may differ with regard to how they perceive interactions with peers as influencing their academic experiences. To enhance this phenomenological approach, I used Mitra's (2004) student voice tool to facilitate stakeholders understanding of gifted student experiences. I believe the rich data resulting from this study provided insight for school and district stakeholders to understand the sociocultural factors that influenced academic motivation, matriculation and level of success for this exceptional group of students.

\section{Case Study}

My selection of a case study design for this research allowed for exploration of a distinct group or subgroup wherein the individuals are a linked because of their commonalities with regard to specific cultural and contextual factors (e.g., AfricanAmerican students in gifted programs). Hays (2004) defines case studies as "research [that] can involve the close examination of people, topics issues or programs" with the primary purpose of enlightenment by answering distinct questions through the collection of details (p. 218). According to Stake (1978), the focus of a case study is the object or 
individual that is likely a member of a population of particular interest to the researcher. In my study each student-informant represented an individual case and were the primary focus of the study. In his critique of proper case study usage among researchers, Yin (1984) elaborates that "as a research strategy, the distinguishing characteristic of the case study is that it attempts to examine: (a) a contemporary phenomenon in its real-life context, especially when (b) the boundaries between phenomenon and context are not clearly evident" (p.2). The flexible structure of case studies make them ideal for exploring individuals or issues in-depth to collect substantial detail and promote understanding (Creswell, 2009; Hays, 2004; Noor, 2008; Yin, 1984, 2003, 2009). Moreover, Yin (2009) notes that case studies are an ideal framework for researchers wishing to explore both simple and complex relationships among and between various individuals and entities and can be enlightening if they are purposefully selected for their relevance to a particular construct. For some gifted African-American students the boundary between academic success and efficacious beliefs are often blurred by the inherent need for peer acceptance and compliance with cultural norms. Multifaceted phenomena such as these are a good fit for case study research, given that its broad design enables the "deconstruction and subsequent reconstruction of various phenomena" (Baxter \& Jack, 2008, p.544) within a particular context. The fluid design of case study research allows for the alignment of two frameworks within this study: academic identity among minority students - to explore how gifted African-American navigate through sociocultural nuances in an effort to establish a clear identity for themselves as students, and how they come to acknowledge beliefs about their own academic ability. In his review of case study research, Flyvbjerg (2006) notes that case studies are advantageous 
in their ability to provide rich detail that resemble participants' personal experiences as they relate to a specific context or phenomena.

Case studies allow for in depth exploration of people and their lived experiences, which aligns with my selection of student voice as the tool to capture in-depth, the sociocultural experiences of gifted African-American students. Stake (1978) affirms that although not readily generalizable, "case studies are useful in the study of human affairs because they are down-to-earth and attention-holding" (p.5). The flexibility of instrumentation and approach of a case study allows the researcher to choose traditional or collaborative case study design in an effort to illuminate participant experiences. In the following section, I describe my rationale for selecting phenomenology framework for this case study.

\section{Phenomenological Case Study}

In my effort to capture the sociocultural essence and peer group experiences of gifted African-American students, I decided to combine the phenomenology and case study methodologies. A phenomenological case study approach seemed appropriate given that each student informant (case) is African-American and the phenomena that connects them is their voluntary participation in gifted education program within the same high school. A case study as a type of inquiry that focus on a specific issue that explores a phenomenon through the analysis of multiple sources (Baxter \& Jack, 2008; Noor, 2008: Yin 2003, 2009). For this study, multiple sources of data were analyzed including transcribed interviews, documents and field notes. Unlinked to a particular methodological style, case studies are therefore open to alignment with the approach that best suits the purpose and nature of the phenomenon under study. The natural flexibility 
of a case study design allows the individual experience of each informant to permeate within a shared academic environment. Sokolowski (2000) suggests that phenomenology "recognizes the reality and truth of...things that appear" (p. 14) and is therefore at the heart of exploring human connection with experienced phenomena. Exploring sociocultural dynamics and peer relationships among gifted African-American students, particularly those who elect to enroll in AP courses, are distinct phenomena that provide valuable insight to stakeholders with regard to program development. In order to capture the experiences of participants effectively, I aligned these two approaches into a phenomenological case study. This collaborative design provided the framework for existing studies such as Sumsion (2002) and West (2013) in which participant experiences were explored in relation to a phenomenon. In the aforementioned studies, researchers explored group (West, 2013) and individual (Sumsion, 2002) participant experiences as they related to a particular phenomenon-each remaining innately focused on capturing the essence experience.

Phenomenological case studies are part of an intrinsic research paradigm. Researchers employ the use of intrinsic paradigm when there is a sincere interest to obtain a better understanding of the phenomenon or case and not simply investigate its divergence from the 'norm' or to build theory (Stake, 1995, 2005). The fluid design and inherent focus on capturing realistic experiences makes phenomenological case study an ideal methodology for my study. The intrinsic nature of my study necessitates the student voice as a data collection tool as it engages reader's interest by soliciting advice from the source of the experience. Mitra, 2004 asserts that we must listen to the voices of students of color and explore their lived experiences. My own lived experience as an African- 
American gifted student and educator have fueled a genuine interest in understanding the sociocultural experiences among gifted students of color-in particular, how these experiences may help to shape the development of their academic beliefs about themselves.

\section{Study Location}

The location for this study is a large urban school district within the southeastern region of the United States. State administrative data indicates that as of 2015 the district serves over 100,000 students attending 173 schools, including 22 high schools, all of which offer AP classes as part of their curriculum. Continuing, there are several schools throughout the state and district designated as consistently low performing. One high school from this Persistently Low Achieving (PLA) group, referred to as North High (a pseudonym), was purposefully selected as the site for this study because of its diverse student body and its recent adoption of open enrollment procedures for its Advance Placement courses.

North High School (NHS) is a Title I school with a diverse student population and is centrally located within the district. According to 2014 State administrative data, school demographic information for the 2013-14 school year shows a total student body population of 1400 the majority of which are students of color- $43 \%$ AfricanAmerican, $42 \%$ White, $10.6 \%$ Latino/a, 2\% Asian, with 2\% of students identifying themselves as two or more races and $0.2 \%$ American Indian/Alaskan Native/Pacific Islander combined. State administrative data show many NHS students are from lowsocioeconomic households with $76.3 \%$ eligible for Free-or-Reduced Lunch. According to state records, the state department of educated designated NHS as a Persistently Low 
Achieving (PLA) school due to its inability to meet its annual measured objective for the last three years. In its continuous effort to exit PLA status and following the diligent work of the school counselor and gifted programs coordinator, Mr. Simmons, school administrators adopted AdvanceState open enrollment process as the primary method for accepting students into gifted programming. The student population at NHS is uniquely diverse with students of color comprising $57.8 \%$ of the population. According to Mr. Simmons (a pseudonym) the goal was to increase Advance Placement (AP) access to diverse groups of students, particularly those of low socioeconomic status and students of color in order to reflect the true diversity of the school. Since deciding to allow its students to participate voluntarily in the AP program, North High School has seen a drastic increase in the number of nontraditional students enrolled in its AP classes and the number of African-American students enrolled has more than doubled. According to 2103-2014 school-level data, school year there were 565 students enrolled in advance level courses, of these 314 received free-reduced lunch rates, 131 are African-American, 76 are Latino/a and 21 identify as two or more races. This is a notable increase compared to the previous school year, during which a total of 168 students participated in the AP program and students of color comprised less than $10 \%$ of the total enrollment. Tables 1 and 2 below highlight the 2013-14 demographics and AP enrollment trends at North High School. 
Table 1

North High School Student Demographics, 2013-14

\begin{tabular}{ll}
\hline Total Number of Students & 1400 \\
\hline Race & $\frac{\text { \% of Total }}{42}$ \\
White & 43 \\
African-American & 10.6 \\
Latino/a & 2 \\
Asian & 2 \\
Two or more races & 0.2 \\
American Indian / Alaskan Native
\end{tabular}

Table 1 shows student population demographics for North High for the 2013-14 school year. The demographics depict the distinctive composition of the student population wherein the percentage of African-American students is slightly higher than that of Whites. North High's central location within the urban school district may have contributed to the diversity of its student population. However, Table 2 shows the diversity within gifted classrooms at North did not accurately reflect the diversity of student population it served.

Table 2

North High School Advanced Placement Enrollment, 2013-14

\begin{tabular}{lcr}
\hline AP Total Enrollment & 565 & \\
\hline Demographic Features & \# of Students & \% of AP \\
\hline Free/Reduced Lunch & 314 & 55.5 \\
White & 337 & 59.6 \\
African-American & 131 & 23.1 \\
Latino/a & 76 & 13.4 \\
Two or Races & 21 & 3.7 \\
\hline
\end{tabular}

As seen in Table 2, the majority of North High students participating in AP courses were from low socioeconomic households, which was representative of the overall student 
population. North High managed to increase the number of low SES students in its gifted courses. School-level documents provided by the AP program coordinator, suggested that there had been an overall increase in diversity from the previous school year; however, the racial composition of North Highs remained aligned with national trends wherein African-Americans were underrepresented.

\section{Sampling Procedures}

Purposeful sampling was used to identify study participants. This was a practical sampling process for identifying the students who were became primary informants for my study. Environmental factors that helped to shape the sampling criteria and overall development of the study included time, paradigm, theoretical framework, interests, and observations (Coyne, 1997). For this sampling method, researchers may choose to identify characteristics to select and then categorize participants based on the parameters of the study (Coyne, 1997). The NHS AP coordinator (e.g., school guidance counselor) identified all male and female gifted students of color enrolled in AP courses. In order to collect deep, rich focused data, three students were selected from the AP coordinator's list based on the following criteria: 1) African-American, (2) enrolled in at least one AP course during the 2015-16 school year, (3) previously enrolled in one AP course during the 2014-15 school year and (4) taken at least one AP College Board examination (passing score is not a requirement). These criteria were chosen because they helped to narrow the pool of candidates to only those with AP experiences emphasized the contextual backdrop and provided a more targeted approach to the study. Given the specific criteria listed only $10^{\text {th }}, 11^{\text {th }}$, and $12^{\text {th }}$ grade students remained eligible to participate in this study as informants. The AP coordinator assisted with narrowing the 
pool of candidates by reviewing class schedules, attendance, and other factors that may conflict with interview sessions. The candidate pool was then narrowed to 10 students who were invited to participate in the study, 3 of whom responded with interest. Each student participant was given the necessary study paperwork (e.g., assent, consent forms) and asked to return it with 14 days of receipt.

In an effort to enhance the experiences these gifted students, I engaged in snowball sampling (Patton, 1990) wherein, each student identified one peer and one trusted school-level adult with whom he or she had a relationship. The assent/consent process described above was followed for all adult and peer informants and each returned their forms before they were interviewed. Each peer and adult informant were asked about their relationship with the student informant, including any observed interactions with peers, as well as academic qualities such as perseverance, work ethic, self-efficacy beliefs and academic performance.

The selection criteria described above allowed for the collection of rigorous data and depth of interactions in order to capture how participants view peers as influencing the development of who they were as students genuinely. These criteria sought to reduce variability, minimizing the contextual differences among participants. I recognized the possibility for inherent bias in this study, however given the relatively small sample population and specific criteria, purposeful sampling was the most appropriate method for participant selection. In his assessment of qualitative research, Patton (2002) noted that all qualitative sampling methods are inherently purposeful due the intentional, indepth nature. For this study, sample size limitations and distinct conditions allowed for the collection of rich, descriptive data during participant interviews that illuminated the 
reality of their experiences (Lester, 1999). This was essential to understanding the interwoven complexities presented in peer relationships and the unique experiences of these gifted students in their academic environment.

\section{Study Population}

Through this study, I explored sociocultural nuances associated with elective enrollment into AP courses for three student-informants. To add depth and richness to informant experiences I also interviewed one peer and one trusted adult; each of whom

the students selected, yielding nine study participants. Student-informants were carefully selected based on specific criteria inherent to this phenomenon and their related experience therein (Creswell, 2009). Participant sampling identification and recruitment criteria were discussed in the previous section and included current enrollment in AP courses and completion of a course exam. Student informants and their classroom peers were each enrolled in AP classes at North High School during the 2016-2017 academic year. Each trusted adult participant was a NHS staff member with whom the student informant regularly interacted (e.g., teacher, mentor, administrator). Table 3 below depicts demographic information about gifted student informants. Table 4 includes demographic information about peers and trusted adults and connects each student to their selected peer and adult. 
Table 3

Characteristics of Student Informants

\begin{tabular}{|c|c|c|c|}
\hline Characteristic & Jayron & $\underline{\text { Alesia }}$ & Victoria \\
\hline & 17 & 17 & 17 \\
\hline \multicolumn{4}{|l|}{ Age } \\
\hline Grade & $12^{\text {th }}$ & $12^{\text {th }}$ & $12^{\text {th }}$ \\
\hline Gender & M & $\mathrm{F}$ & $\mathrm{F}$ \\
\hline Race & $\mathrm{AA}$ & AA & AA \\
\hline Years attending NHS & 4 & 4 & 4 \\
\hline \# HS attended & 1 & 1 & 1 \\
\hline \# Years in AP courses & 2 & 3 & 4 \\
\hline $\begin{array}{l}\text { Identified as } \\
\text { gifted/talented in ES }\end{array}$ & No & Yes & Yes \\
\hline $\begin{array}{l}\text { Enrolled in district gifted } \\
\text { courses in ES and/or MS }\end{array}$ & No & Yes & Yes \\
\hline Cumulative GPA & 2.689 & 3.827 & 3.969 \\
\hline
\end{tabular}

Note: Pseudonyms were used to protect informant anonymity. 
Table 4

Trusted Adult and Peer Informant Demographics / Relationships

\begin{tabular}{|c|c|c|c|}
\hline \multicolumn{4}{|c|}{ Characteristics of Peer Informants } \\
\hline Characteristic & $\underline{\text { Hanah }}$ & Deveon & $\underline{\text { Ava }}$ \\
\hline Age & 17 & 17 & 17 \\
\hline Grade & $12^{\text {th }}$ & $12^{\text {th }}$ & $12^{\text {th }}$ \\
\hline Gender & $\mathrm{F}$ & M & $\mathrm{F}$ \\
\hline Race & AA & AA & AA \\
\hline Enrolled in AP courses & Yes & Yes & Yes \\
\hline Student informant relationship & Jayron & Alesia & Victoria \\
\hline $\begin{array}{l}\text { \# Years known student } \\
\text { informant }\end{array}$ & 5 & 6 & 3 \\
\hline $\begin{array}{l}\text { How acquainted with student } \\
\text { informant }\end{array}$ & MS & MS & HS \\
\hline \multicolumn{4}{|c|}{ Characteristics of Trusted Adult Informants } \\
\hline Characteristic & Mr. Moore & Ms. Michaels & $\underline{\text { Mr. Simmons }}$ \\
\hline Role & Teacher & Teacher & $\begin{array}{l}\text { Guidance } \\
\text { Counselor }\end{array}$ \\
\hline Content / Detail & $\begin{array}{l}\text { English / AP } \\
\text { Language Comp }\end{array}$ & $\begin{array}{l}\text { Business / } \\
\text { Technology }\end{array}$ & AP Coordinator \\
\hline \# Years employed in district & 6 & 3 & 12 \\
\hline \# Years employed at NHS & 6 & 3 & 6 \\
\hline \# Other schools employed & 1 & 0 & 2 \\
\hline Race & $\mathrm{AA}$ & $\mathrm{W}$ & $\mathrm{AA}$ \\
\hline Student informant relationship & Jayron & Alesia & Victoria \\
\hline $\begin{array}{l}\text { \# Years known student } \\
\text { informant }\end{array}$ & 1.5 & 4 & 3 \\
\hline $\begin{array}{l}\text { How acquainted with student } \\
\text { informant }\end{array}$ & $\begin{array}{l}\text { Befriended by } \\
\text { chance / In-class }\end{array}$ & In-class & $\begin{array}{l}\text { Assigned as } \\
\text { counselor }\end{array}$ \\
\hline Type of relationship & Mentor & $\begin{array}{l}\text { Teacher / } \\
\text { Mentor }\end{array}$ & $\begin{array}{l}\text { Counselor / } \\
\text { Mentor }\end{array}$ \\
\hline
\end{tabular}

Note: Pseudonyms were used to protect informant anonymity. 
Table 3 shows demographic information about each student informant including age, gender, race and gifted enrollment status. Cumulative grade point averages (GPA) were provided to highlight each informant's current academic achievement level as a gifted student. Table 4 organizes demographic date for both peer and trusted adult informants including features such as the number of years attending North High and employee role within the school building. Table 4 also connects each peer and adult to the student informant with whom they have a relationship.

\section{Participant Confidentiality}

It is the responsibility of the researcher to ensure confidentiality, particularly in qualitative studies wherein participants divulge personal and detailed information about their lives and experiences. Given the in-depth scope of qualitative studies, "researchers face a conflict between conveying detailed, accurate accounts of the social world and protecting the identities of the individuals who participated in their research" (Kaiser, 2009, p. 1632). Confidentiality includes avoiding connections that may identify participants and taking appropriate steps to protect data and documents (Ritchie, Lewis, Nicholls, \& Ormston, 2013). This includes anonymity, which refers to efforts made to protect participant identification beyond members of the research team (Ritchie et al., 2013).

In order to effectively highlight student voice and capture the details of participant experiences, interviews served as the primary source of data collection. I used pseudonyms to ensure anonymity of location and participant identity. As a part of the transcription process, I digitally recorded transcripts and secured them on a password- 
locked computer. Transcribed interview records were stored electronically on both a locked computer and an external password-protected drive.

Prior to beginning the investigation, I secured permission from university Institutional Review Board (IRB), district and school-level officials, school administrators and parents/guardians to conduct participant interviews. Each participant received a letter and consent form explaining the study purpose, parameters and requesting permission to participate in interview sessions. Parents/guardians of minor participants were required to give their permission by signing assent and consent forms. For the purpose of data triangulation, as well as to further ensure the protection of personal information, member checking process were used in which electronic copies of individual interview transcripts were provided to each participant. I provided each participant with the opportunity to review and provide feedback on their transcribed interview and I made changes (e.g., redactions, corrections, clarifications) accordingly.

It is the responsibility of the researcher to ensure study participants are cared for during throughout the duration of the study — in particular for qualitative research, given the flexibility of its structure and the potential for unexpected issues that may arise (Ritchie et al., 2013). As a part of informed consent, researchers must notify potential members that their participation in this study is voluntary and that electing not to participate is not punitive (Ritchie et al., 2013).Each study candidate was required to submit ethical documents prior to participation in the study. The consent form included pertinent information regarding study purpose and parameters, protocols, confidentiality, data collection and use, as well as participant requirements. In addition to consent forms, for student members under the age of 18 , assent forms were also required. 
Parent/guardian signature was required on these forms prior to participation in the study.

In addition, I invited participants and their parent/guardian to contact me with any questions or concerns they had for the duration of the study.

\section{Data Collection}

For this study, I merged two methods of inquiry, phenomenology and case study, which together may include a variety of data sources such as documents, artifacts observations and interviews (Baxter \& Jack, 2008; Lester, 1999). The use of multiple data sources (e.g., student informants, peers, adults, documents) may generate large amounts of data; however, cross-case analysis of informant data increased the credibility of the study (Baxter \& Jack, 2008; Lester 1999; Yin, 2003). For this study, the emphasis on student voice allowed the use of interviews as the primary source of data collection, as well as the analysis of publicly accessible school district documents. Interviews provided in depth information on participants' perspectives and were a prominent qualitative data source (Creswell, 2009). Qualitative researchers may analyze documents as another source of data to support interviews and observations. For my study documents were used to gather academic data including grade point average, enrollments, class schedule, gifted/talented identification, and assessment scores. Analyzing multiple data strengthens triangulation, findings and implications of a study (Baxter \& Jack, 2008; Miles, Huberman \& Saldana, 2014; Yin, 2003), thus enhancing the reliability of the study.

\section{Interview Protocol}

I conducted interviews with three African-American AP student-informants at North High who elected to participate in these classes through the open enrollment process. For each gifted student selected, I also interviewed one peer and one trusted, 
school-level adult, yielding nine study participants. Interviewing a peer and a trusted adult provided a broader perspective into the sociocultural and academic experiences of focus student. Additionally, it provided rich data that may uncover hidden connections between peers and academic self-worth. It was expected that conducting tiered-style interviews (e.g., gifted student, peer, trusted adult) would uncover related themes that provided a realistic perspective of participant experiences. I conducted each interview separately. Interviews lasted for 60 to 75 minutes. All interviews occurred during the same month of the fall semester and there were no noted logistical or attendance issues.

Each interview was facilitated using a semi-structured protocol (see Appendices A, B C) consisting of scaffolded questions, addressing general background information, academic history, social and cultural norms formatted in both definitive and open-ended style. Student informant interview protocol (Appendix A) contains approximately 25 questions, trusted adult (Appendix B) and peer protocols (Appendix C) contain 10 questions each. Questions were developed using information gathered extant literature, research questions and similar interview protocols from a study on peer group influence on achievement and self-efficacy in academic contexts (Usher, 2009) designed to facilitate open dialogue with participants regarding their academic experiences. The semi-structured style provided the "openness" necessary to elicit the complex sociocultural dynamics, peer interactions and personal experiences of gifted AfricanAmerican students. A diverse question style allowed me to establish rapport with participants for the development of topics that unexpectedly emerged and supported the collection of rich data with a distinct "insider's perspective" (Leech, 2002, p.665). Peer and trusted adult interviews had similar protocols both semi-structured designed to elicit 
information about their interactions with the student informant. The added depth from peer and adult informant interviews provided a broader picture of student informant experiences. However, it was imperative that students voices were amplified of the African-American gifted students themselves to better understand what their strengths and struggles to enable educators to provide holistic (e.g., social, cultural and academic) supports to ensure their success.

To ascertain efficacy beliefs in mathematics among middle school students, Usher (2009) engaged a system wherein students were ranked based on average scores from an assessment that measured efficacy at different levels. As part of her study assessment, Usher (2009) allowed students to rank themselves on scale 1 (low efficacy) to 6 (high efficacy) across various tasks and then used mean scores to lead her discussion. To better understand self-efficacy beliefs among student informants, I incorporated a modified version of Usher's (2009) protocol, allowing student informants to rate their ability to complete tasks often associated to gifted coursework. As part of the interview protocol, student informants were asked to rate themselves on a scale of 1 to 10 , where 1 represented no confidence in completing the task and 10 represented full confidence in successfully completing the task. This rating system was not used for peer and adult informants, however they were asked to share their perspectives on the student informant's efficacy factors. A detailed discussion of efficacy data can be found in Chapter IV.

\section{Document Analysis}

I used document analysis as part of my study also. Document analysis is the process of evaluating documents to gain knowledge that supports a thorough 
understanding of participants, settings and background information. Bowen (2009) suggests that researches should perform document analysis to synthesize data and align it to the research questions and purpose of the study. Documents provide empirical strength for qualitative research because "they already exist in the situation; "they do not intrude upon or alter the setting...nor are they dependent on the whims of human beings..."

(Merriam, 2002, p. 13). Researchers locate and define documents that are relevant to their study and uses them to facilitate the synthesis of data and to preserve the context of the study (Patton, 2002).

For the purposes of this study, I collected and analyzed public documents, such as school, district and state level administrative data on demographics, school achievement, Advance Placement program (e.g., enrollment trends, demographics). In addition, I reviewed electronic records of student-informants in order to acquire information about scheduling, academic grades, grade-level enrollments and standardized assessment data. These documents provided essential information on the background and current statistics of the school and district settings and provided the contextual basis for participant experiences. Although interviews were the primary sources of data, document analysis facilitated the sense-making process of interview data and offered support of informant responses..

\section{Data Analysis}

Interviews were audio-recorded and transcribed on a personal computer. Following transcription, interview data were coded using deductive coding. Saldana (2013) suggested that researchers use deductive coding to ensure codes are aligned with study frameworks. Thus prior to conducting interviews, I undertook both structural and 
deductive coding in order to create a list of codes as a means to organize emerging themes into categories based on peer relationship features, self-efficacy and academic identity theories. According to Saldana (2013), structural coding is questioning-style coding method used to categorize data associated with broad themes and is used during first cycle coding to organize data by categorizing it into segments that may be efficiently compared. Continuing, Saldana (2013) notes that this coding is particularly useful in practitioner research and provides for illumination of "child and adolescent voices [which] are often marginalized, and coding with their actual words enhances and deepens an adult's understanding of their cultures and worldviews" (Saldana, 2013, p.91). This type of coding enhances the true meaning for adults because of its student-centered focus on capturing their actual experiences. For second cycle coding I used data theming or thematic analysis (Boyatzis, 1998), in order to identify intersecting ideas that emerged from participant responses. I then organized the themes into subcategories that were utilized during cross-case analysis (refer to Table 7 and Table 8). Each of these coding methods highlighted participant perspectives, emphasized student voice and provided a unique richness to the data. I uncovered themes that captured participant challenges surrounding the social and cultural nuances that influenced their ability to identify efficacious beliefs and solidify their identities as students. In particular, I uncovered themes among participants associated with factors such as identity development, peer interactions, cultural norms and beliefs, and feelings of inclusion and exclusion.

In addition to informant interviews, I conducted document analysis to obtain demographic information on each student and peer informant, as well as academic data such as grade point average, transcripts and assessment results. The information obtained 
from documents was used to supplement data not shared during interviews and provided additional details that enhanced informant perspectives.

\section{Credibility}

Within qualitative studies, the researcher must establish credibility of the study from the perspective of the participants themselves. Since the goal of qualitative research is to explore social experiences of participants, the position and empathy of the researcher may inherently influence the results of the study. Creswell and Miller (2000) identify several methods of validity within qualitative studies, including extended fieldwork, member checking, triangulation, negative evidence, positionality, collaboration, detailed descriptions and peer debriefing. In determining which method to select, researchers must consider the scope, context and parameters of the study.

Given that my study was designed to elicit the experiences of students within a specific context, member checking was be used to enhance credibility. Member checking allowed participants to review study data for authenticity and representativeness typically during triangulation process of data analysis (Cutliffe \& McKenna, 1999). Creswell and Miller (2000) define member checking as the return of "data and interpretations back to the participants in the study so that they can confirm the credibility of the information and narrative account" (p.127). Participants are experts in their own perspective and can legitimately identify discrepancies in the portrayal of their own experiences (Trochim, 2006). It is the responsibility of the researcher to inquire about the accuracy of participant data and corresponding evidence and that any additional comments are included as part of the final product (Creswell \& Miller, 2000). After I transcribed the interviews, I shared a digital copy with each individual informant. Participants were allotted time to review 
transcripts and submit revisions. No informants submitted revisions to transcribed interviews.

Triangulation is a process during which researchers analyze qualitative data across to develop themes (Creswell \& Miller, 2000). Triangulation is a viable source of credibility because instead of depending on a single data source, the researcher is required to use various sources of information to minimize bias and increase the validity of the data. Typically, researchers will search their sources for repetitive information for the merging into categories and subcategories that enhance the richness of the data. Mathison (1988) notes that analyzing data in this way strengthens a study against critical review, minimizing contradictions about the phenomenon under study. For this study, data triangulation will stress the contextual factors surrounding sociocultural experiences of gifted African-Americans as this is the common thread that connects the informants to one another - the shared phenomena. To enhance the credibility of my study further, I facilitated the triangulation process by conducting informant interviews, analyzing documents, and member checking. Document data were cross-referenced to ensure stability of student informant responses and elicit academic information

\section{Transferability}

Establishing trust is a prominent task of researchers seeking to increase the validity and reliability of their study. This is especially true for qualitative researchers who are responsible for affirming the transparency (i.e., trustworthiness) of their study by acknowledging all potential biases and providing detailed information about study protocols, contexts, participants and activities (Denzin, 2009). Transferability is one component designed to increase the validity of study results. The transferability of a 
study is the extent to which the reader perceives the results as applicable to alternative contexts and locations (Trochim, 2006). Although transferability may enhance study trustworthiness and validity, the degree to which a study is transferable is not the burden of the researcher, but tasked to those wishing to apply study results to outside situations. In their analysis of research protocol, Healy and Perry (2000) insist that within qualitative paradigms, "....applicability or transferability are to be the essential criterion for quality" (p. 601). Given the sample size of my study, the applicability of its results to other school locations, populations or contexts is unlikely. The triangulation of interviews, documents and member checking along with the methodological detail discussed in this chapter helped to enhance transferability and transparency of my study.

\section{Researcher Positionality}

As discussed previously, qualitative researchers seek to establish credibility and transparency in their efforts to highlight phenomena. This is an essential component for qualitative studies, especially for those concerning issues of race and culture. Race and culture are prevalent factors in educational research yet due to the inherent sensitivity and multifaceted perspectives, researchers should actively engage in analysis and acknowledgement of their own biases and position. Milner (2007) notes that "it is important that researchers possess or are pursuing deeper racial and cultural knowledge about themselves and the community or people under study" (p. 388) and in doing so suggests a framework for establishing researcher positionality in educational research that includes several interrelated features: researching self, researching self in relation to others and reflection and representation. Milner's framework guides researchers to 
consider and explicitly acknowledge their position on race and cultural views, in an effort to anticipate possible barriers and pitfalls when dealing with participants of color.

In light of my position as an African-American gifted program educator and former AP scholar, it is possible that my positionality will either distort or inform my perspective and objectivity. Milner (2007) insists the race and culture of study participants be considered throughout the research process and failure to do so leads to precarious "exploitation and misrepresentation of individuals and communities of color" (p. 392). Researchers must be self-aware and acknowledge the multifaceted perspectives they bring to their research (Milner, 2007). For this study, my inherent biases are worthy of exploration to ensure my commitment to remain objective in this research effort and so as not to conflate participants' personal experiences with my own. My positionality enhances the contextual perspective as a researcher, providing a sense of comfort for interview participants who may be otherwise reluctant and guarded to share their experiences. Within the study, my role as a researcher is multifaceted. As such, I explored this more in depth in the methodology section of this chapter.

My study focuses on Advance Placement African-American students and how they navigate sociocultural influences in the development of academic self-worth. Given this context, I must acknowledge and reflect on my current roles as an African-American educator, and parent of a gifted student, as well as my prior experience as a gifted student, seeking to understand how each of these influence my perspectives on race and culture and my role as a researcher. I understand that as a gifted elementary school student, seeing no one else that looked like me in my classes for two years, inadvertently impressed upon me issues surrounding race and racism. Similarly, throughout my tenure 
as an educator I have taught numerous gifted courses, observing only a handful of African-American students per class. As a result of these and other similar experiences, as a researcher I was driven to explore the experiences of other gifted African-American students to understand how issues of race, culture and societal norms influence them academically. Yet to do this effectively, I am tasked with establishing firm credibility for my study, including carefully navigating and acknowledging my preconceived notions about race and culture which may influence my positionality as a researcher. Below I discuss how I used Milner's framework to address potential issues concerning my positionality and as a guide to navigate potential issues that may arise.

As an African-American researcher, it seems logical that my exploration of African-American students would be unpretentious, given the obvious similarity of race and the assumption that our cultural experiences are similar. However, Cruz (2001) disagrees, stating that researchers of color who investigate participants of color must be intentional in their ability to "develop alternative spaces and methodologies for the study of their communities" (p.658) in order to depict the experiences of participants accurately without convoluting them with their own (Milner, 2007). Throughout the duration of this study, I engaged in self-research to remain aware of how my experiences and biases may surface, actively reflecting upon how these may be similar or divergent from those of my study participants. To facilitate research of self and self in relation to others, Milner (2007) suggests the use of narrative discourse to accurately represent participants' experiences followed by counter-narrative to highlight alternative or opposing views shared by other participants or the researcher. Continuing, Milner (2007) argues that through narrative and counter-narrative "emphasis and value are placed on knowledge 
construction, on naming one's own reality, and on the multiple and varied voices and vantage points of people of color" (p. 391). This type of discourse does not silence the topic of race, but instead pushes it to forefront of the study bringing clarity to every voice aiming to be heard.

\section{Summary}

Chapter III discussed methodology and research design of the proposed study to explore the extent to which peer interactions influence self-efficacy and academic identity of gifted African-American students. Qualitative methodology was selected because it allows for an in depth exploration of complex issues (Creswell, 2009; Miles, Huberman \& Saldana, 2014), in particular the sociocultural experiences of this unique group of students. I chose a phenomenological case study as it is a methodological that explores "processes, events and activities" (Creswell, 2009, p. 177) that align individuals within a similar context. Capturing the voices of gifted minority scholars whose choice to challenge themselves and strive for academic excellence may be complicated further by navigating the nuances of sociocultural conformity. 


\section{CHAPTER IV: RESULTS}

In this study, I explored the perceptions of African-American Advanced Placement students. The following research questions were used to guide my study: (1). What are the perceived influences of peers on the development of self-efficacy and academic identity of African-American students enrolled in Advanced Placement courses? (2). How do African-American students in Advanced Placement courses perceive interactions with peers and cultural ideas towards achievement, as influencing their overall academic experiences in Advanced Placement courses?

In this chapter, I discuss the results that emerged from my analysis of the collected; the centerpiece of which were nine individual semi-structured interviews: one interview each with three African-American AP students (referred to as studentinformants); one interview each with three in-school peers; one interview each with three trusted adults. The individual student informant selected a peer and trusted adult at the conclusion of the interview. The semi-structured interview protocols (See Appendices A, B, C) were designed to illicit experiences from participants, peers and adults within four categories: (1) general/background information, (2) self-efficacy, (3) peer group relationships (AP and traditional peers) and (4) academic identity. The semi-structured protocol allowed for unscripted questioning, which provided some measure of flexibility in the exploration of participant experiences. 
Discussion of data and analysis are organized into five sections. The first section provides a profile of each student informant. The second describes each participant's path to becoming an AP student. The third section explores perspectives on studentinformants' relationships and interactions within peer groups (e.g., AP, non-AP). The fourth section reviews the self-assigned efficacy ratings for each student informant (Table 3) and highlights student informant perceptions of their own efficacy, as well as observations from peers and adults. The final section describes key components of student informant' academic identity (Table 4) and the perceived influence peer groups may have on identity formation. A summary of informant responses grouped by categories and subcategories created during first cycle structural coding, along with thematic schemes generated from second cycle data-theming coding. These are organized and displayed in Tables 6 and 7.

\section{Informant Profiles}

In this study, each student-informant shared their academic, social and cultural experiences as a self-identified gifted student of color. Then, each participant selected an in-school peer and trusted adult, each of who were contacted and asked to share their perspectives on the student-informants (refer to Table 3). I assigned pseudonyms to all informants regardless of their role in order to protect their identities.

\section{"Jayron"-A Diamond In the Rough}

Jayron is a 17-year-old senior who has attended North High since the starting the ninth grade and is on track to graduate with his class. Jayron is the youngest of three children. Despite his parents' divorce, they established with their son that pursuing higher education was a formal expectation. Jayron's brother and sister are enrolled in college 
currently, leading by example and leaving him as an 'only child' in the house. During our interview, I took note in my observations of his repeated shifting in his chair and nervous laughter with some topics that appeared to make Jayron cautious and unsure of himself. Despite this, he valued the importance of higher education and this was evidenced by discussion of his plans to follow his sibling's examples and attend college. Currently, Jayron is enrolled in two AP courses, AP English and AP Environmental Science.

When asked to describe his personal attributes, Jayron responded that he was uncomfortable in doing so, stating, "Uh, I guess I'm more relaxed than others (laughs)...I guess uh...it's weird talking about myself (laughs)." Jayron conceded that he has no one in his life that he admires or looks to as a role model. Throughout our conversation, he provided few positive comments about himself and often deflected instead to what others have said about him. For example, when asked about his qualities as an AP student, in my notes I referenced the extended pauses, nervous laughter and his eyes looking downward each time before Jayron briefly responded about others' comments about him. Jayron highlighted that during parent conferences teachers often shared that he was respectful, nice and thoughtful.

\section{"Alesia"-Momma Duck}

Alesia is a 17 -year-old senior and Governor's Scholar ${ }^{3}$ promoted to North High from a middle school math-science technology (MST) magnet program. Alesia's parents are divorced and she lives with her father and his girlfriend. She has a large blended

\footnotetext{
${ }^{3}$ The Governor's Scholar program is an academically competitive state-funded six-week summer program for high school seniors designed to promote economic and civic leadership through targeted university curriculum, project-based learning and a college campus experience.
} 
family, including four siblings and other live-in children she affectionately refers to as her "soon-to-be stepsiblings." Alesia characterized herself in this way:

I'd say a mix between extraverted and introverted because I can be a homebody but I also enjoy spending time with my friends outside of school and I also like to talk to different people, new people, like I enjoy my job. I work at shoe store so I associate with people I enjoy.

In my observations, I noted that Alesia's body posture is confident as she speaks, sitting upright, making frequent eye contact and smiling. Her discussion of her goals indicates a focus on long-term career plans and the necessary steps she must take to achieve them. After graduation, Alesia plans to attend a four-year university and pursue a degree in journalism. Ms. Michaels, her trusted adult, described her as a big sister who holds everyone accountable and tries her best to keep them focused and on task in class. During the 2016-17 school year, Alesia elected to take two AP courses: AP English and AP U.S. History and dual-credit Humanities course. She has realistic expectations for herself and understands exactly what is required in order for her to be successful.

\section{"Victoria"-I Can and I Will}

Victoria, who is 17 years of age, has attended North High since her freshman year. Victoria is the youngest of three children and resides with both parents. Her siblings have moved out and she is currently the only child still living at home. Victoria is a Governor's Scholar also who enjoys school and is a self-described optimist that surrounds herself with like-minded peers: 
I'm a really happy positive person. I'm organized. I try to like keep everyone else happy and positive and I guess I don't let things way down on me. I'm workoriented. Yeah, they're positive people and we always try to get our work done. We don't like to dwell on too much, we like to get things done.

Victoria proudly claims her aunt as her role model for her ability to overcome adverse circumstances and persevere to achieve success:

The circumstances she grew up in were worse I guess than the ones I grew up in and she still managed to go to college, get three degrees and have a successful job and raise her kids as a single mother, so I admire that.

She uses her aunt's story as motivation to push through academic challenges and personal trials to continue working towards her goal of enrolling at a four-year university. Victoria acknowledges that she is a socialite and that, if it were not for her friends, school would be boring. However, her social nature has not deterred her academic focus, as she is enrolled in three gifted classes this year (AP Biology, AP English and AP U.S. History) and a dual-credit Humanities course. Victoria works hard, encourages others, and continues to persevere towards her goal of attending college.

\section{Journey to Giftedness}

This section includes highlights from each participant's discussion about their choice to enroll in AP courses voluntarily. Participants revealed how motivation from personal obstacles, along with encouragement from friends and trusted adults, were all influential factors in their decision to take gifted classes. 


\section{Jayron}

A former student athlete, Jayron was a member of the North High football team during his first two years. His athletic career was short-lived when the last of several concussions he received put an end to his participation in all contact sports. When asked to reflect on his ability to persist through difficult tasks or overcome challenges, Jayron shifts in his seat, looks downward, sighs, laughs nervously, and reflects on his recovery from his last concussion. Later, when asked if he enjoyed school, he again reflected on his recovery, acknowledging the academic struggle that followed:

Yeah, yeah it's not that bad (laughs). At first it was a little rough, but now it's not that bad. Like around the time I got hurt. I got a concussion and that kind of played in everything, like my grades dropped and everything so it got a little rough.

Mr. Moore, business teacher and mentor, recalled Jayron's emotional dissonance following his unexpected departure from the football team:

Now from an emotional standpoint I know that he [Jayron] struggled mightily with not being on the football team and wanting to be there. I think it was an embarrassment that he wasn't there because he may have suggested to people that he was 'less than' or not capable. I think he took a couple - a few serious - a few pretty serious hits that knocked him out. I mean when you concuss that's serious. It's not like you can make that up so I do believe it disturbed him...

Following his recovery period, Jayron enrolled into his first AP course and although embarrassed to admit it, he was not prepared for the course work and expectations: 
'Cause it's a...like I'm kind of new to taking AP classes - the pace its totally different and I'm trying to adjust to the pace. I took AP English... and that sucked (laughs a little). I didn't do too good [sic.] in that. I think I passed with like a D or something.

Despite this initial poor performance, Jayron continued his AP matriculation adding additional AP and dual-credit courses to his course load. Jayron's resilience as a gifted student although admirable, was not intrinsically driven and through various conversations, it was revealed that his motivation was influenced by classmates and friends significantly.

\begin{abstract}
Alesia
Alesia's parents prepared her for the challenges of advanced level coursework by enrolling her in the MST program at a local middle school. As a high school freshman, Alesia participated in Advanced Program ${ }^{4}$ classes in elementary school which are courses designed promote creative thinking and independent learning among students identified as gifted-talented as determined by district criteria including standardized assessment scores. Alesia's parents selected her path to gifted education, but as she grew older, she learned to find the value in accepting challenges is the reward that comes with persistence. Her best friend, Deveon, spoke candidly about her commitment to academic achievement:
\end{abstract}

\footnotetext{
${ }^{4}$ According to 2015 district-level data Advanced Program is a pool of courses offered to academically gifted students primarily identified by benchmark score on a standardized assessment; input from teachers and parents may also be considered as supplemental criteria. Participation in Advanced Program may begin in intermediate grades.
} 
She puts her schoolwork before everything. She don't go out that much. She'll be like "aw naw I got to do all this" ya know. Or she'll uh....and she's like really working on trying to like.... she really wants to go to the University of ------------. She's really focused on trying to get there-she's working on a bunch of stuff.

A self-proclaimed writer, Alesia told me English was her favorite subject and that she has learned to accept critical feedback as a way to improve her writing:

My best subject is English because I enjoy writing and there's always room to get better at it and I'm always up for feedback like constructive criticism in my writing because that's what I want to do when I'm older I want to be a journalist so I like English.

A review of Alesia's transcript shows her grades and are continuously exceptional, suggesting that her participation in Advanced Program classes in elementary school was an appropriate match for her aptitude and skill level.

\section{Victoria}

Victoria revealed that her elementary school experience introduced her to the lack of diversity often associated with gifted education:

In elementary, I went to a predominately White school. Middle school was predominately White school. Girl scouts—White girls I guess. And they'll never understand, never get you. It's really a one-sided relationship—you're really putting in all that effort to be their friend and fit in.

Victoria admitted that her failed attempts to befriend White classmates in elementary school led to her strong association with peers more similar to herself (i.e., African- 
American) and the confidence to speak out about social inequities and racial discourse, particularly among gifted peers.

During her freshman year at North High, Victoria attended both advanced program and honors level classes. She elected to enroll in AP classes for the first time during her sophomore year after continuous encouragement from the school counselor, Mr. Simmons, the trusted mentor that she identified. During the interview, Mr. Simmons indicated that he believes Victoria exemplifies the qualities of an AP student, and noting her leadership abilities, willingness to learn and desire to be successful. He stated:

She [Victoria] stands for success. She is goal-oriented. She is an achiever. She wants to be the best at whatever she does... She brings her AP peers to our "Road to College" club and she leads by example. So she shows them that she wants it that she wants to be successful, she wants to go to college.

Matriculation through AP courses provided the backdrop for Victoria's acceptance into the Governor's Scholar program and according to friend, Ava, awakened her academic motivation:

...she does it for herself, like she really wants to do good, so she really does good in school. And like she's not really like a student she's a scholar, you know like there's a difference—like students just come to school, but scholars actually want to be here and do good.

For Victoria the decision to enroll AP classes not only provided access to the rigorous college preparatory coursework and critical-thinking skills, as her friend described it also 
shapes her identity as a scholar and cultivated the I can-I will attitude that would become part of her persona.

\section{Peer Group Relationships: "Who You Wit"”}

Adolescence is a transformational period potentially influenced by variety factors, in particular peer groups (Chavous, Hilkene, Schmeelk-Cone, Caldwell, Kohn-Wood \& Zimmerman, 2003). A goal of my study was to explore how African-American gifted students perceive peers as influencing their academic self-concept and identity formation. Each student informant maintains peer relationships with gifted and traditional peers, yet the extent of their interactions with each type of peer subgroup and corresponding reasons are attributed to a variety of factors.

\section{Jayron}

When Jayron began his high school career, he enrolled in honors ${ }^{5}$ courses primarily, and this is where many of his peer relationships and groups originated. His interactions with some of these peers has decreased due to his decision to enroll in AP courses. He reflected on one honors classmate in particular, who did not support his AP decision:

Yeah I used to have this one. He graduated last year. He'll tell me like to get out of it-he was saying 'cause it's just too hard and stuff. But I was like well if that's what you think ya know (scoffs)....I mean I don't — that's you that's not me.

\footnotetext{
${ }^{5}$ Several years prior to this study, North High School received its Persistently Low Achieving (PLA) designation as the result of a state audit of its academic progress and administrative effectiveness. In the administration's effort to improve school culture and exit the school from priority status, a student-centered culture shift ensued. As a part of these, comprehensive classes (or "regular" classes) were eliminated and all students, no referred to as "scholars" were enrolled in honors and/or advanced courses accordingly.
} 
Jayron said that he still associates with honors classmates and that his AP peer group has grown as his participation in those classes increased. He reveals that his gifted peers are supportive of his choice to take challenging courses seemingly because "they want to see me do better I guess." However when asked to consider if his the majority of his selective peer group are AP or non-AP, he acknowledges they are evenly split, "so it's pretty much....I'm kind of in the middle." He attributes this divide to that fact that since last year his schedule has been a mix of both honors and gifted classes.

Nationally, the number of students of color participating in AP programs is significantly lower when compared to their White counterparts; particularly for AfricanAmerican males, who make up the largest deficit in the national achievement gap (U.S. Department of Education, 2009a, 2010). Several of the gifted classrooms at North High could easily be mistaken for comprehensive classrooms - the level of diversity in each are strikingly similar. Jayron provides contrasting views of this reality as he discussed the diversity in two different AP classrooms:

Okay uh...in my English class there's a lot of minorities I think—well actually I take that back, there's probably like 6 or 7 of a class of like 31 and in my environmental [science class] there's 2-2 Black, 3 Black people.

Jayron revealed that the majority of his friends are African-American and the only traditional peers he associates with are a handful of his former teammates. Although he has no apparent explanation of how this came to be, some of his closest friends and biggest supporters are a part of his African-American gifted peer group, including his best friend Hana. During our individual interviews, Jayron, Hanah and Mr. Moore all agree that Jayron and Hana's relationship resembles that of siblings. Mr. Moore describes 
Hana as the mature, goal-oriented older sister who constantly checks on her less organized, seemingly complacent younger brother. Although they do not have any classes together this year, Hana and Jayron have been friends since the eighth grade. When asked to describe Jayron as a student, Hana noted:

He works hard. He gets down on his self [sic.] a lot. Sometimes he lets himself get to him, like his thoughts-- cause he feels like he's not able to do it. He kind of gives up on his self a lot.

Hana admitted she is concerned about her friend's wavering self-confidence. She believes in its weakened state, that his high-achieving peers influence his confidence negatively:

Yes I think [they] influence him, but does he act on that influence?

Sometimes not. But then he'll see that other students are doing good and sometimes says "I just can't" and he'll give up and sometimes he'll ask for help like he's supposed to.

Further, she acknowledged her frustration with Jayron when he gives up, but she continues to encourage him and he eventually comes around.

\footnotetext{
Alesia

The transition to gifted courses increases the level of rigor and challenges students to think critically, equipping them with the problem-solving skills that will better prepare for college (Callahan, 2003; Kyburg et al., 2007; Princeton Review, 2017; Viadero \& Johnston, 2001). Alesia revealed that she associates with a small group of peers all of whom are African-American gifted students. She explained her reasons for affiliating primarily with AP peers:
} 
Because we see the benefits that it can give us for college so we-all of us want to go to college so we just try our hardest in the AP classes instead of taking easier that we may be too smart for anyways. With my AP peers it's easier for us to talk to each other and motivate each other 'cause we are in the same classes. With my non-AP peers I feel kind of bored to talk to them because there's not much to talk about because they don't really relate with what I'm going through when it comes to school I guess.

Alesia revealed that during her freshman year, her peer group was a mix of gifted and traditional students and it was during her transition into gifted courses that an unintentional shift occurred:

'Cause we weren't in the same classes. They may not have understood everything I was going through. That's just like my freshman year, I wasn't in AP but I had two friends that were and they had a lot of stress on them. I like my social studies class it's pretty cool and it's advanced. I didn't really know that— the complete difference between the AP classes freshman year. So when I joined AP junior year I understood what they were going through, but I also understood what my other friends were going through which they did stop being my friend--- not on purpose but because we weren't in the same classes.

When asked if her gifted peers supported her choice to take AP courses Alesia described a memorable conversation with a friend:

Some of them support it and one was saying before I started taking AP she was completely saying not to do it because she had already took them and she said no 
don't do it, it's going to be horrible, you play sports, you're not gonna be able to do it, it's hard. Then if you don't pass the test you won't even be able to benefit from it, but I still took them anyways, I thought it would be a good experience and it was.

She continued, explaining why her friends that are not in gifted courses are unable to influence her:

Because, I don't want to say I don't value what their opinion is, but I feel like this is what I want to do. I don't just want to be in regular classes. So even if they did say something to me like "you're putting too much work on yourself or you don't have to do all that" I'm still gonna feel like I have to. For one, my dad always pushes me to so nobody's gonna change that and sometimes I feel like my nonAP friends don't really understand why I'm in AP classes cause they've never tried it.

Alesia shared that some of her non-AP peers supported her decision to take gifted classes. However, she indicated that they did not understand why she voluntarily increased her workload and frequently questioned her dedication and sanity. Alesia acknowledged difficult coursework could become stressful at times and shared her current gifted peers' perspective:

They say it's a lot of work but it is definitely hard. It's just we have to motivate each other and ourselves to do the work. Some of them get overwhelmed but others don't. The frustration comes with all the work 'cause it's a big load of work that's basically what they say. 
According to her teacher, Ms. Michaels (See Table 3), Alesia is viewed as the matriarch among her peer group as she is often tasked with getting her classmates refocused whenever they are sidetracked. This is not something Alesia discussed during her interview; however, Ms. Michaels repeatedly boasted about her no-nonsense attitude towards her complacent classmates:

Positive or negative Alesia is hilarious 'cause she's so-I don't even think she would know to be self-aware about this-Alesia gets fed up with people when they're not doing what they're supposed to do. So she was in here with [a student]—listen — (laughs) — and he'd be snoozing in the corner or you know not having his stuff and she'd be like "get it together-would you please, this is ridiculous!" She was definitely taking on that older sister kind of role with him and being like "are you kidding me right now" because she saw him making excuses and acting in ways she knew was not acceptable and she knew he was capable of more....she'd be like "that's foolish" or "that's ridiculous" or "could you please stop talking" or "would you get back on track" or "wake up." So she's pulling everybody else along with her in this very sort of momma duck kind of way_like everybody get in line come on we're doing this and she was very motivational and she stayed on them.

Ms. Michaels admits proudly that she is one of Alesia's biggest cheerleaders. She revealed that admires her ambition, determination, perseverance and her pragmatic way of thinking and doing without a sense of entitlement. Ms. Michaels continued that Alesia's classmates look up to her and her personal code is inspiring to them: 
And Alesia's own personal code is like "uh-uh if you can do it you do it and there's no excuses." Even if it's done maybe not as well as you wanted it to be or you don't think it's - that's what I love about Alesia is even though she has this self-doubt, she does it. She doesn't make excuses and say well I wasn't sure so I didn't do it....she does the work she doesn't let that stop her from making the attempt which is exactly the perseverance you need to be successful in anything and she has that.

For Alesia, issues with peer group influence seem to arise when she compares herself to AP peers and believes she cannot measure up. However, it seems that Alesia is most influenced by the expectations set forth her parents and by the expectations she has created for herself. She states:

I get motivated because I think about the...my parents' experience with school and they always tell me that I have to be better than them so that motivates me to do better in school and actually get a job that I want to get and I just think about my future a lot so that motivates me.

\section{Victoria}

Similar to other student-informants, Victoria reported she most of her friends are AP students. She explained the reason for her selection of gifted peers was that they had more in common with her than her traditional peers.

I guess freshman year I had balance I had AP classes and I classes with non-AP students and you really can see the difference I guess. AP students, well my AP 
peers they're just like me. Motivated always ready to do what they got to do, but they still want to have fun on the side and talk. So I like that that they're balanced.

Continuing Victoria admitted that she did not realize the shift in disassociating with her non-AP peers during her freshman year-it just happened:

Well in non-AP classes I guess students really don't care. We're just trying to get out of that class. Trying to still do our thing and get out—-listen to music sleep talk—really in non-AP really it's busy work that's all it is. It's really just you gotta do this, just do it and we're good so and that really didn't help the student and that really told the student that hey you really don't have to put much work in to anything you can still pass. So those students start to get lazier and lazier to the point where they just stop caring. They would leave and go do what they wanted to do because they really had....there was nothing challenging them. And then with the AP students you see that someone is challenging them, they're putting in work they never skip school, never. We get asked to skip school all the timenever. Because we got classes we gotta go to we got things we got help we need after school anyway. But then when you don't take AP class there's nothing challenging you and then you just don't care anymore and you're like it's not good for nothing it's not helping me and you just go. And I needed some people that were just gonna keep me motivated keep me positive keep me ready to do what I had to do rather than dragging me down with them to laziness.

When asked about the racial composition of her peer group, Victoria acknowledged that it is comprised of predominately African-American students: 
In elementary, I went to a predominately White school. Middle school was predominately White school....you're really putting in all that effort to be their friend and fit in with them but with other Black people it's effortless you are just automatically understood and it's that mutual if you need something I'm gonna help you out. You gotta ask rather than you gotta make that effort to really be their friend. You gotta straighten your hair you gotta try to buy some blue contacts and make up and didn't even touch on the topic. But, with my Black friends it's so much easier they can help me out they understand what's going on they're like yeah I've been through that too. I told them I told my friend I had to take a cold shower the other day 'cause my water heater went out. She was like "girl yeah I been through that too". And I told a White friend she was like "what, what does that mean? What do you mean you had cold water? Why didn't you just go take another shower in another room?" And I was like that's not really how it works. It's just you gotta explain stuff with them rather than someone feeling you and getting it.

Victoria's peer group perceptions are different from that of other studentinformants. She does not allow her peers to influence who she is as a student $—$ she hears what they say and perseveres. Victoria instead listens to peers' criticism about her choice to enroll in AP classes, complaints about her working all the time (e.g., "acting White") and even compliments on her hard work:

I'm too worried about stuff. I'm always looking at college stuff or doing work staying in school or going to talk to some teachers. I'm always talking to teachers and counselors and stuff. And they're like "you're always doing that stuff' uh 
"why you never skip with me" or "why you never go do this with me; why you still doing homework right now?" or "why you so worried about it?" like “you always doing this stuff; I guess let's just go have fun let's just go do this; That's a big question I always get or why don't you just chill out, let's just chill— like it'll be middle school, let's just go chill. No I don't want to for real. That's a big question. And some of them are like that's inspirational you know that you're doing what you need to do or they wouldn't really say it like that but they'll say in like I guess more informal ways um that like you just doing what you gotta do you know.

Victoria attributes her peers' criticism to the fact that they are focused on different goals: "I guess that maybe that we focus on different things I guess our focus is more on work and school and stuff like that and for non-AP students it's on maybe home stuff. “ Nevertheless, she listens and their comments only propel her forward towards success.

\section{Self-Efficacy as a Gifted Student: "I Think I Can Do This"}

This section summarizes data collected on student informant efficacy beliefs from several perspectives. As previously mentioned, efficacy protocol and rating scale were modified from Usher's (2009) study on efficacy in middle school mathematics, wherein students utilized a scale to rank themselves from low to high efficacy. Table 5 shows selfidentified efficacy ratings for each student informant based on a questions regarding confidence-level with accomplishing specific tasks associated with AP coursework. Data on student informant self-belief, task confidence and perceived peer impact are highlighted, as well as peer and trusted adult perspectives on these topics. Table 5 shows informant responses to efficacy rating questions regarding confidence with rigorous 
tasks. Informants were asked to rate their level of confidence on a scale of 1 to 10 with a specific task and then were asked to explain why they selected that score.

\section{Table 5}

Student Informant Self-Efficacy Rating for AP Tasks

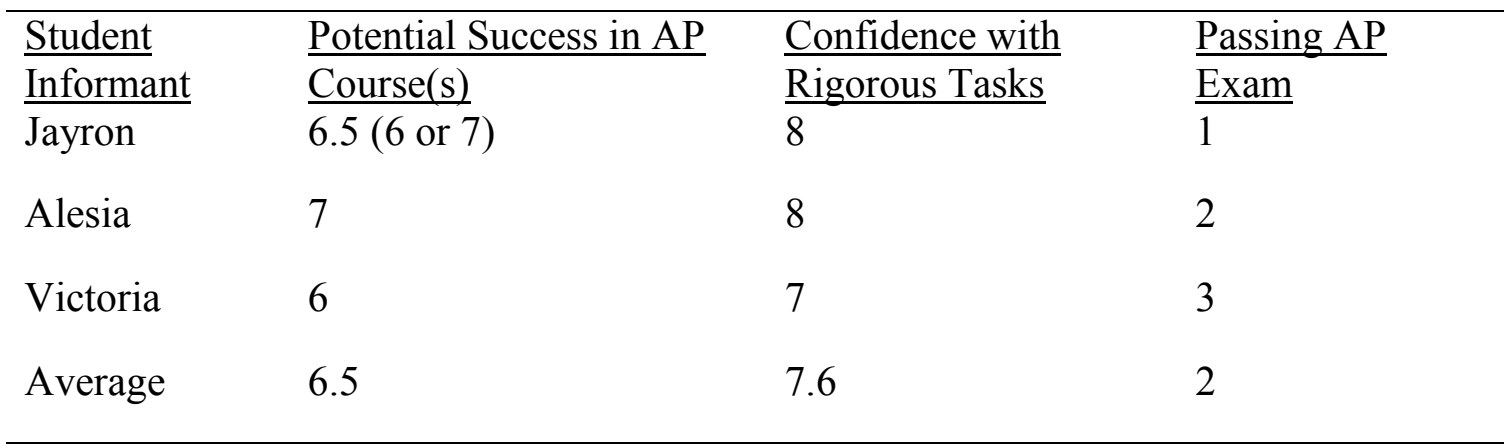

Note: Rating scale 1-10, where $1=$ no belief in self with completing task; $10=$ full belief in self with completing task

Data from Table 5 demonstrates that students are fairly confident their ability to compete difficult coursework and succeed in gifted classes. Despite their belief that they can successfully complete classwork, students are much less confident in their ability to pass the culminating AP exams.

\section{Jayron}

Jayron is a 'diamond in the rough' who, by all appearances, defies the negative social and educational stereotypes assigned to African-American males. On the surface, Jayron appears well-suited for gifted courses, yet while engaging in conversation with him it becomes apparent that he possesses minimal academic confidence and low selfefficacy. Researchers insist that students lacking self-belief often avoid difficult tasks and show minimal engagement and effort (Bandura, 1997; Schunk, 1995; Schunk \& 
Swartz, 1993). When asked to rate his potential to succeed in gifted classes, Jayron was assured that he would do well:

Interviewer: If you were asked to rate yourself or rate your potential to succeed in your AP classes on a scale of 1-10 - where 1 is I don't think I will do very well and 10 is I'm going to be awesome in my AP classes, where would you be?

Jayron: Uh, I'd say like—I'd say like a 6 or a 7.

Interviewer: A 6 or 7. Okay why do you say 6 or 7 ?

Jayron: 'Cause it's a...like I'm kind of new to taking AP classes - the pace its totally different and I'm trying to adjust to the pace

Jayron is more confident in his ability to complete AP work and perform well in his gifted classes:

Interviewer: So using the same scale 1 to 10,1 is low 10 is high — how do you rate your confidence now in doing AP work?

Jayron: I feel better.

Interviewer: So what would you give yourself on a 1 to 10 ?

Jayron: I'd say like an 8.

Interviewer: An 8, okay. So why do you give yourself an 8 now?

Jayron: This year I'm a little more prepared. I know what's about to come and I know where I'm supposed to like- how much work I'm going to get and I can actually do the homework and stuff like that. 
Interviewer: Alright. So you're organizing your time a little better and...

Jayron: Yeah it's just time management that's all it is-I got to get better at it.

Later it was revealed that his improved confidence to complete assignments came from classes where he received assistance from high-achieving peers. Yet when it came to individual rigorous tasks such as exams, his confidence level decreased significantly and self-doubt ensued:

Interviewer: Using the same scale of 1 low $10 \mathrm{high}$, rate your confidence in passing the AP exam at the end of the year.

Jayron: I'd say 1. (laughs)

Interviewer: 1, really? Wow! So have you passed an AP exam before?

Jayron: No.

Interviewer: What could make you feel more confident about taking an AP exam?

Jayron: Uh I don't know, I guess actually preparing myself for it. 'Cause last year I just, I really didn't care about it last year. I just took the test so I could get the grade for that class, but this year I don't know, that test was pretty hard...

When asked if he is self-motivated, Jayron affirms that he is and illustrates with an example: "Yeah nobody really stays on me about my grades so that's why I was like I gotta do it ya know — they're [teachers] not gonna...so I'll set reminders on my phone about homework and stuff." However, Hana has a different perspective on her friend's motivation in the classroom: 
I do believe he's focused. Organized, maybe not (laughs). He tries to motivate himself but it's like a big cycle—-he motivates his self and then he gets down and then he doesn't do the work sometimes and then I'm like, "how do you expect to do better if you don't do the work?"

Hana spoke about observing Jayron as he selected which students to pair up with for group work during an AP class. She indicated that he purposefully chose to work with students who he knew were performing well academically because he knew they could help him be successful.

Although Mr. Moore was Jayron's identified school-level adult, Ms. Michaels acknowledged her familiarity with Jayron and in agreed that he was "very respectful, very well-mannered and very well-kept" and both acknowledge his low self-confidence and poor academic motivation. His business teacher and trusted adult mentor, Mr. Moore, noted that Jayron desired success, but constantly sought the reassurance of his high achieving peers to validate his wavering confidence because he is afraid to fail.

When asked 'what do you think about something?', Jayron' immediate response until he hears from someone else is 'I don't know' - and it's not a thoughtprovoking 'I don't know' it's a 'let somebody else answer it and I'll formulate a response based on what someone else said.' He has not demonstrated or exhibited leadership qualities although they're there, I truly believe that they're there, he has not taken hold to demonstrate them. So I think there's a fear that Jayron has of failing more than anything and he's embarrassed by that failure so he chooses rather than to try to just act like paint on the wall and try to blend in. 
The idea of model similarity wherein over time adolescents become more similar to the groups with which they frequently interact (Hymel, Comfort, Schonert-Recihl \& McDougall, 1996; Schunk \& Pajares, 2001), does not apply to Jayron in the context of this study. He has instead become co-dependent on his peers, looking to them to validate his confidence on a task or to reaffirm his own thoughts before speaking them aloud. Jayron lacks confidence in his own ability to succeed and struggles to identify endearing about himself. Yet without hesitation, he praises his friends for their intelligence and influence, stating, “... they're very smart-- I have really smart friends. They're uh really focused on getting to school and I like being around that, like the positive energy." Moore has high hopes for Jayron, but is concerned that reliance on peers is making him complacent and ill-prepared for the academic rigor of post-secondary education. Moore acknowledged that in the classroom setting he has observed first-hand, Jayron's avoidance of difficult assignments:

Jayron is very well-kept, very mannerable, very respectful, but he's also very lazy to be quite frank and he will find the shortest way to get something done and the least benefit from it...But I don't think Jayron is working near as hard as he could.

Jayron's low academic confidence and low self-efficacy are evident to peers and adults around him. Glimpses of his self-doubt are also prevalent in the self-reflections he shares of his experiences inside gifted classrooms. Despite his lack of self-belief as a gifted student, the personal obstacles he overcame and his lingering battle with self-motivation and confidence, informants familiar with indicate that Jayron is well liked and possesses limitless untapped academic potential. 


\begin{abstract}
Alesia
I observed that at the start of our interview that Alesia appeared shy and nervous in the presences of unfamiliar company however, underneath the surface was an intelligent, confident young lady. Alesia admits that she likes school, but that without her friends class would be less enjoyable, even boring. Alesia's admits her favorite class is AP English and that her weakest (her least favorite) is AP U.S. History-not surprisingly this coincides with her strength and confidence in those courses.

My best subject is English because I enjoy writing and there's always room to get better at it and I'm always up for feedback like constructive criticism in my writing because that's what I want to do when I'm older I want to be a journalist so I like English. My weakest is AP US History or any kind of history. I'm just not good at it at all. And a lot of things we learn kind of mess with my brain and make me upset like the American history and all that so I don't really like learning it — but it is history so I guess I really have to.
\end{abstract}

The wavering confidence across specific subjects has not deterred Alesia's overall confidence in her ability to complete work:

Interviewer: Using that same scale of 1 to 10 , how would you rate your confidence in doing your AP work?

Alesia: With that I'd give myself an 8 because I try to push myself and I do push myself to do better-it's just what I think I need to work on is time management and not being so lazy. 
Interviewer: So you give yourself an 8 which is pretty confident. Is there anything that could make you feel a little more confident about your work even though that's pretty high? Anything that could push it to a 9 or a 10 ? You said time management so what could help you? Cause definitely in college that's something you're going to have to get with so what could help you to get better with that?

Alesia: I think having classes that I'm actually interested in will definitely help me because I wouldn't be lazy about it I would be motivated to do better in the class. Because in high school they just make you take certain classes and I know in college they do to but I'll have more freedom to be motivated in certain classes.

Although confident about completing in-class assignments, Alesia clearly doubted her ability to pass an AP exam considering her previous unsuccessful attempt. Alesia shared a few initial ideas she believed would improve her chances of receiving an AP exam score of 3 or higher and how her perspective on the importance of the exam has since shifted:

Interviewer: what's your confidence level for the exam?

Alesia: Two.

Interviewer: Really a 2?!

Alesia: Yes (laughs)

Interviewer: Alright, so then now I can ask you this question—what could make you feel more confident about taking these AP exams? 
Alesia: Um, better studying skills. The thing is we don't know what's going to be on the AP exams so my teachers prepare me as much as they can and then I see the test and it's nothing I've ever seen before. Last year was my first time ever taking the AP exams and it was just a big knock on the door because I didn't know what was going to be on the other side--- yeah so I' not really confident in them anyways because they're so intimidating — like "oh in order to get your credit in college you have to get a 3 or every school they might not give you any even if you pass.” So I think on the inside I don't really care if I pass, I'm just doing it because it looks good basically.

Interviewer: It looks good for...

Alesia: ...for colleges when they're looking at you to see that you actually challenged yourself in high schools....

Deveon, Alesia's friend since middle school, describes her as a "hard worker" who is "organized, focused and motivated" and he believes she is an ideal fit for gifted classes: "She's really smart so she [most definitely] fits in with the AP classes." Alesia attributes her self-motivation to the continued reflection on her parents' educational hardships, resilience and their push for her to be successful.

I get motivated because I think about the...my parents' experience with school and they always tell me that I have to be better than them so that motivates me to do better in school and actually get a job that I want to get and I just think about my future a lot so that motivates me. 
Although her source of motivation was personal to her, she chose to share it with teacher and mentor, Ms. Michaels:

She's just....she's talked so many times about—either one-on-one or when we're looking over college essays just about how she looks at her parents lives and not for good bad or otherwise but just knows---but she questions a lot if they had finished college how different would their lives be-cause they went but they didn't finish because I think her mom got pregnant with her I think while her parents were in college. And that's something she goes to again and again is always wonders like how different things might have been if they had been able to finish that. And that is deep for her that is a really true driving force

Ms. Michaels was adamant that Alesia does not hold a negative view of her parent's choices, but instead considers it a reason to set high expectations for herself and hold steadfast to the belief that she must be more successful than they were:

And again it comes up so frequently—and not in a big way, well I'm sure she does but—I don't think she even notices how much she brings it up but it's big for her-- parents and not in a negative way. Her parents are a big influence thoughand she wants and any kid does and as any parent would want they want to do better that their parents - do more or have more have a better life. She definitely sees that. Her parents obviously support her from what I can tell. She needs to like take over the world.

For Alesia, the embedded motivation manifested itself into the perseverance needed to remain focused during challenging tasks: 
In my government class last year we had a debate and paper to write and I don't... I didn't usually have opinions in government or history classes so it's hard to have a debate paper if you don't really have a natural opinion. So I was going through it-I cried so much and I had to write my essay and I did this like two days before it was due I think because I didn't know what to write about or what to actually say. I don’t really know... I didn’t know where to get my information from. I didn't know which websites were trustworthy, it was just really hard. After it was all over and my team, because the teacher made us do different teams-my team actually did okay and it was just a big weight lifted off my shoulders because that debate paper was horrible....It was a C. And I was proud of myself because I was expecting a $U$ [to fail].

At times when Alesia struggles through coursework, her self-belief begins to decline, she feels overwhelmed and stressed and begins fearing that she will fail her classes. She often confided in Ms. Michaels when she reached her breaking point:

Interviewer: Anything else that concerns you most about being an AP student?

Alesia: Not passing the class itself. Sometimes I feel like that might happen, but as it turns out when I get my little progress report I'm doing fine. It's just a stress thing - the stress is a concern 'cause I stress out a lot anyways.

Ms. Michaels: But she did have times when she would get frustrated. When she hit a point of being overwhelmed she would lose it and just say "this is stup..." not this stupid but this is like, she's not a kid that would say "stupid"-she would always say like “I'm done; I'm just can't do this, I'm done." And sometimes there 
was tears and sometimes she just got really overwhelmed so we'd have to go out in the hallway—and it was never about -as it is with kids—it was never about what was in front of her...just feeling like she was so overwhelmed.

She pointed out that aside from not passing the AP exam, failing a course is one her biggest concerns and at these moments, positive self-talk the most helpful tool: "I have to constantly tell myself that I have to do it and that it's going to be over soon. I have to give it my all and I have to get it done." Alesia acknowledged that for rigorous assignments she will occasionally seek help from her AP classmates. She continued that the help she receives from classmates is not always beneficial and she may resort to consulting online resources, textbooks or the course instructor assistance.

Alesia's efficacy beliefs are generally positive and are driven by her strong intrinsic motivation fueled by her parents' push for academic achievement. When faced with adversity Alesia may experience moments of self-doubt and fearfulness, but she digs deep to find the strength and resilience to propel herself forward.

\section{Victoria}

I noted in my observations that in her discussion of experiences, Victoria's selfdescribed optimism keeps her attention focused on the tasks in front of her. Her friend Ava noted that Victoria is positive, organized, self-motivated and will always complete her work even if she complains about it. That is, except when it comes to those classes she considers 'complex' that reach beyond the realm of her creative learning style and challenge her comfort level. Victoria candidly admitted she prefers abstract courses such as psychology and art that allow for creative expression over concrete classes like science and math. She stated: 
The science is so complex. Every little thing has another little thing that makes it do what it does. And there's like equations to every little thing, why it does what it does, why the thing makes it do what it do does and it's just too much, too complex.

Her dislike of these courses has not adversely affected her academic performance, as she maintains the ability to persevere through difficult tasks, maintaining a 3.8 cumulative grade point average. When faced with rigorous assignments, Victoria admitted to seeking assistance from others:

I guess I'll ask the teacher to further explain it to me. I'll look to peers to see if they know what this is. I'll look over in the book, go to Google see what can help out and once I developed a further understanding then I'll try it out. If I still don't get it then I'll talk to a counselor someone who can find ways for me to understand. Or go talk to another teacher, teachers have different teaching styles so someone else could show me what I need to do.

Likewise, instead of feeling discouraged by high-achieving classmates, Victoria's efficacy improved because she was inspired:

It makes me want to get on my stuff. Or I'll ask them: 'hey how do you know all this? What resources are you using? What are you doing that I'm not doing? What are you doing that I should be doing?'’

I noted that the confidence to request help and the skillfulness to know which viable resources to turn to are important features of Victoria's self-motivation. Ava also 
observed a positive shift in her Victoria during her association with some highly motivated peers:

And when she was around them [Governor's Scholars Entrepreneurs] she always talked about how she was motivated to do better because you are around people who are a little bit better than you, you know what I'm saying - it's kind of like so when she's around smart people she pushes herself to be smarter or try to equal to them. She's like 'man I don't understand how they do it.' And then she's like keeps going.

When asked to rate her level of confidence in her potential to succeed in AP classes, Victoria revealed her need for deeper learning as a source of concern:

Interviewer: Rate your potential to succeed your overall ability in your AP classes on a scale of 1 to 10 .

Victoria: Um... about a 6.

Interviewer: A 6, okay. Can you tell me why you picked a 6 ?

Victoria: Um, I guess because I'll do the work if you ask me to do the work. I'll turn in stuff, I'll do all I need to do but, the issue is that sometimes I'm not understanding things and we don't talk about them we don't do none of that we just move on and I'll try to work on it at home but the resources aren't there or the things I need aren't there so I'm still lost then we all just got to move on. If I stay on a topic too long then I'm even deeper lost so I gotta move on when they move on. 
Victoria's dissatisfaction with the surface learning and pacing of the AP courses may have influenced her belief that she can be successful here. Her confidence in completing gifted coursework was slightly higher which Victoria credits to her ability to listen and follow directions:

Interviewer: So, on a scale of 1 to 10 , rate your confidence in doing AP work.

Victoria: Mmmm....(pauses)...7, yeah.....7.

Interviewer: Okay, why do you say a seven?

Victoria: Um, for most of my classes, it's pretty easy to do the work. Um, you just got do the work I guess pay attention and do what you gotta do. So I'm pretty confident in that I'll get the work done, I'll make time for it.

Interviewer: What would make you feel more confident in getting the work completed?

Victoria: If I understood every little thing that I was doing. If I was completely confident that I would pass every test that a teacher gave me.

Evidence, such as that provided by Ava, suggests that Victoria embodies a scholarly attitude as she continues to refer to her need for deeper understanding of contentcompleting and submitting required assignments is not enough.

Despite the fact that Victoria has already passed and received college credit for two AP exams, she rated her confidence level only slightly higher than the other studentinformants who have not passed exams. As she explained her reasoning, the scholarly poise once again resurfaced coupled with a bit of frustration: 
Interviewer: rate your confidence in passing the exam at the end of the year

Victoria: About a 3.

Interviewer: About a three? Okay. So why not so confident?

Victoria: Um, I guess because I tend to forget the content and I usually only pass when I am really passionate I guess about the topic and I feel like that teacher has taught me everything I needed to know and has fully prepared me

Interviewer: Okay. So um, have you passed an AP exam before?

Victoria: Yes.

Interviewer: What could make you feel more confident about passing the AP exams?

Victoria: Um, I guess if the teacher had... if there was more structure. Sometimes there's not enough structure to the class and we're here then we're there then we're back to this then we're at something else and like it's not really progressing I guess it's sort of just bouncing everywhere; they just throw pages in a book at you rather than actually taking the time to teach the content to you like giving you examples showing you the best ways to do it how to get through it rather than just throwing pages at you or handing you a paper and saying try this, do this or and then getting a good grade even though it's wrong. Like I know that I've turned in papers that were completely wrong and I got an A on it for effort. And then on the test I flunk 'cause I really don't know what's going on. So really what would help would be if teachers really wanted you to learn rather than just do the work. 
When discussing her exam scores, Victoria's response to the question suggested frustration. She was critical of the teacher's only awarding effort grades for rigorous assignments and assigning what she categorized as "busy work" in lieu of meaningful learning experiences.

Mr. Simmons, school counselor, acknowledged Victoria's self-motivation as one of several qualities that made her an excellent choice for the AP program:

Yeah Victoria is intrinsically motivated so she wants be different and she wants to like I said earlier, she wants to be successful. She knows that to be successful college has got be a major part of her life. So I think that a big part of her academic career at North High has been to push herself, to make good grades, to do well on the ACT, participate in different clubs and extracurricular [activities] so that she can do well academically-- and thus, be able to go to college.

Mr. Simmons' comments provided a glimpse into Victoria's high school experience including the academic and post-secondary goals she set for herself. During the interview, Victoria reflected on time when her motivation fueled her resilience and shared this experience:

I think a time is when I guess nothing was really working in my favor. My brother was going through stuff, my sister... like no one in my family I could really reach out to. Mom was sick, she was really sick I couldn't even talk to her and my dad he was always at work so there was no one really to talk to. My peers they were busy with their own lives so I feel like that was mid junior year when everything was like tumbling and I just.... we had so much work to do so I think that was I 
time where I really had to push through. We had ACT studying stuff and trying to get a job, trying to find people to help you out with college applications and what you need to do next year 'cause nobody tells you what you need to do next year.(laughs)..., if you don't ask no one's gonna tell you. So, I tried to make relationships with teachers and counselors people that could help me out so that's when I was really self-motivated.

Similar to other student informant, self-efficacy for Victoria includes motivation and confidence; both of which fluctuate only slightly depending upon the context of the situation or task at-hand. Victoria's persistent desire for deeper learning frustrates her scholarly nature, yet she remains resilient and focused on her goals.

\section{Academic Identity: African-American and Gifted}

Academic identity is a progressive, multidimensional concept that includes social, cultural, psychological, cognitive, and developmental components that intertwine, creating distinct persona for each individual. Among gifted students, the formation of an identity often rests with their level of intrinsic motivation and their belief in their own ability to succeed at difficult tasks. For African-American students solidifying their role as students is complicated further by racial identity. That is, not only how they see themselves as learners, but more importantly how they view themselves as part of racial group that is bombarded with negative stereotypes, particularly with regards to educational attainment. For gifted African-American students, the racial identity component adds depth to the vastly complex task of discovering who they are as learners. Table 6 shows student informant academic identity features identified by the participant themselves, peers and/or trusted adults. 
Table 6

Student Informant Summary of Identified Academic Identity Factors

\begin{tabular}{|c|c|c|c|c|}
\hline Student & Racial & Gifted & $\underline{\text { Peer }}$ & Personal \\
\hline Informant & Identity & $\underline{\text { Feature }}$ & $\underline{\text { Related }}$ & \\
\hline Jayron & $\begin{array}{l}\text { Yes, acute } \\
\text { awareness. }\end{array}$ & Potential & Co-dependency & $\begin{array}{l}\text { Dependent } \\
\text { external } \\
\text { motivation }\end{array}$ \\
\hline Alesia & $\begin{array}{l}\text { Yes, pressure to } \\
\text { excel. }\end{array}$ & $\begin{array}{l}\text { Self- } \\
\text { motivation }\end{array}$ & Comparison & $\begin{array}{l}\text { Parent pressure; } \\
\text { 'momma duck' }\end{array}$ \\
\hline Victoria & $\begin{array}{l}\text { Yes, vocal on } \\
\text { issues. }\end{array}$ & Aptitude & $\begin{array}{l}\text { Improves } \\
\text { Motivation }\end{array}$ & I can and I will. \\
\hline
\end{tabular}

The factors shown in Table 6 were derived directly from student-informant, peer and trusted adult responses collected during the interview. As part of the coding process, informant responses were themed and the key components that captured personal attributes of an informant's identity were selected and placed into the table. Categories listed horizontally at the top of the table aligned with theoretical frameworks (e.g., efficacy, academic identity, peer interactions) and were derived during coding process. The second column of Table 6 shows how each informant identifies race as part of their identity and how they have responded to experiences with race in their gifted classrooms. This table contrasts varying features of informants' identities including personal and peer-related factors, which provided additional insight into who they were as students and how they interacted with peers.

\section{Jayron}

Since adopting AdvanceState open enrollment policies, enrollments for students of color has significantly increased at North High. However, Jayron is one of only two African-American males in his two AP classes during the 2016-2017 academic year. 
When asked to describe non-academic experiences in gifted classrooms, Jayron revealed he was not the only one to recognize this vast disparity:

Jayron: Uh...I don't know. I feel like I'm kind of looked at differently. Like when I walk in. 'cause it's just...it's not that many Black [males] in my classes. Yeah the principal...Ms. Henley (pseudonym)...came in and she was like "I'm glad you're taking it." She only came in and talked to me and I was like "uh, okay."

Interviewer: Were you the only black male?

Jayron: ...Yes...actually there's one other one in each class.

Jayron cited that encounter with his principal as a negative experience that made him more acutely aware of his race within the classroom — a factor of his academic identity of which he was obviously aware but, seemingly had not paid much attention to until now.

Jayron was uncomfortable discussing his role as a student or those qualities that best described him as a student. Instead, he seemed to defer to what other people have said about him:

Jayron: Mr. Simmons always says that the teachers call me a leader and stuff, but I don't know. I don't really see it.

Interviewer: What do you see your best qualities as a student?

Jayron: I guess I won't quit on things and uh I don't know...talking about myself is weird.

Jayron's reluctance to speak positively about himself (e.g., self-esteem) throughout the interview suggests an overall lack of confidence and low self-concept (Pajares, 1995; 
Schunk \& Pajares, 2001; Cokley et al., 2011). Jayron's closest friend Hana admitted she becomes frustrated at times with his self-doubt and complacency:

He tries to motivate his self but it's like a big cycle - he motivates his self and then he gets down and then he doesn't do the work sometimes and then I'm like, 'how do you expect to do better if you don't do the work?'

Mr. Moore, teacher and mentor, reflects on his concerns for Jayron's poor academic motivation in the classroom:

There's very little self-motivation in James and that the part that I've been trying to help encourage....he's so underperforming and has so much potential and so much talent that and he's just not pushing himself so he's going to fall short—if this pattern remains he going to fall short in life with achievement just because he won't allow himself to push that one step or two steps further.

Those around Jayron have an alternate perspective of his struggles that he does not see, at least not completely. Despite the obstacles, Jayron attempts to increase his academic performance are fueled in part by the value for education his family instilled in him. Jayron noted that he frequently falls behind with the rigorous pace of gifted classes, yet he is determined to improve his grades:

...They're [my grades] alright right now... Right now my GPA for the past six weeks is like uh a $3.5 \ldots$ but like my cumulative is like a 2.5 so I'm trying to get that back up....nobody really stays on me about my grade so that's why I gotta do it ya know...they're [teachers] not gonna. 
Efforts towards improving his grades are also motivated by what seems to be a fundamental component of Jayron's academic identity as an African-American gifted student — codependency. Jayron is continuously comparing himself and his work to that of his AP classmates, particularly those he deems more intelligent or capable than he is. As Hana mentioned previously, this codependency on classmates has become an inherent part of his identity to the extent that he uses it as a means to gauge the level of effort he will put forth towards a task. At times, these comparisons, coupled with the intrinsic selfdoubt, move this capable gifted student into moments of hopelessness:

Yeah, they be doing better than me. (laughs). Yeah sometimes, like when uhsometimes when—like the class moves fast sometimes so it's like when everybody else is on pace and on track and everything and I'm just like ok like that — and everybody else is understanding and like when I ask a question everybody's just like “what?!"-like I'm ya know, like I feel like I'm beneath them or behind them. I don't know. Sometimes I feel like I shouldn't even be in there.

For Jayron, peer competition - the need to compare himself to his classmates constantly became a subconscious process - an everyday function in his role as an AP student and an inherent component of his academic identity. Establishing an academic identity is challenging simply considering the complexity of factors such as race and gender. Jayron's task is further compounded by his fluctuating intrinsic motivation, poor selfconfidence and codependency on both peers and adults. 


\begin{abstract}
Alesia
Although completely unaware, Alesia likely began developing her academic identity at an early age when introduced to gifted-style programming during elementary school by testing into advanced level classes. The advanced program track continued through Alesia's junior year when she elected to participate in AP courses. This was due to the insistence of Mr. Simmons, her school counselor. Alesia states:
\end{abstract}

I was always advanced ever since elementary school but the real actual AP started my junior year...because my counselor told me that I needed to be in them because I was doing so well in the advanced classes.

This early start in higher-level courses has helped to provide Alesia with critical problemsolving skills and academic confidence she needs to persevere in gifted classes. Alesia's communication style, although informal, is articulate and poised as she responds thoughtfully to each question. When asked to describe positive personal experiences in AP classes, she acknowledged her value for learning real-world connections and crosscurricular applications:

Positive things are that I'm actually learning something that can help with me with my real original life, like at home maybe or I can actually teach my parents things at times. And I remember last year a lot of my classes were actually connected so this class would help me learn about this class. Last year, I was in AP Psychology and we were learning about the studies and how sometimes in statistics they use studies you know make someone learn, well you know analyze the study; so I was also in AP Stats and they were talking about the same thing in those two classes. 
Alesia attributes her value for educational attainment to her parents' continuous reminders of their mistakes, which translate into high expectations for her. Following her expectations, Alesia post-secondary goals are to attend a four-year institution where she plans to pursue a major in journalism. Likewise, she eagerly boasts about her favorite subject and explains how it coincides with her future career plans: "My best subject is [AP] English because I enjoy writing and there's always room to get better at it... When I'm older I want to be a journalist so I like English." Alesia is fueled by her desire to achieve, focusing on the result and knowing how to set realistic goals that will help her get there. Those who know her best, comment on her commitment to her future academic plans:

Deveon (best friend): She puts her schoolwork before everything. She don't' go out that much. She'll be like 'aw naw I got to do all this' ya know. She really wants to go to [State University]-- she's really focused on trying to get thereshe's working on a bunch of stuff.

Ms. Michaels (teacher, mentor): Alesia is incredibly dedicated. She is determined. She knows exactly what she wants to do. As far as like I don't know if she knows exactly what she wants to do to study in college but she knows she's going to college. She knows she wants to be successful and she knows what that takes. We've talked a lot about it — this year especially she's had me read over her college essays- - her application essays and I've written her a couple letters of recommendation. And she's very aware and has placed this burden on herself that college is everything. So she's very motivated. 
Alesia acknowledges her self-motivation and discusses how her continuous reflection along with strong advice from her parents push her forward:

I get motivated because I think about the...my parents' experience with school and they always tell me that I have to be better than them so that motivates me to do better in school and actually get a job that I want to get and I just think about my future a lot so that motivates me.

Ms. Michaels, who has known Alesia since her junior year, describes her as a student who will achieve success because she know what it takes to get there. Most noteworthy are her comments about Alesia's motivation:

Motivated?! Absolutely she is self-motivated! She is self-motivated. She is inquisitive. She wants to know and that's what I think the hallmark of any AP student should be. It's not how smart are you it's how much do you want to learn. And so that's her to a "T"- she wants to learn because she wants to do well, she wants to be successful....and I think that's pretty much her.

As is common with most students, Alesia's confidence is stronger in certain times and weaker during other times. During times when her confidence is shaken, it is her fierce academic motivation that provides the additional support, particularly when she is being compared to her gifted peers. It is at these moments that Alesia's confidence is at is weakest point and self-doubt sets in:

Alesia: I know sometimes I'm being compared to other AP students and if I feel like they are doing better than me it kind of brings me down.

Interviewer: So, it makes you be harder on yourself? 
Alesia: Yeah, hard on myself or I feel not confident.

Alesia calibrates her own achievement gauge based on the performance of her gifted peers. A measure she admits causes her to feel out of place in AP classes, particularly during those times when she believes she does not measure up:

If I see another student doing better than me or actually answering the questions correctly in class it does motivate me to do better, but it also brings me down like 'man that's not what I had on my paper; why do I not understand what they're saying?'

Ms. Michaels recalls moments when Alesia experienced lack of confidence resulting from comparison to peers:

I think she doubts herself in those AP classes way more than she needs to. I think she has this feeling that she's not an AP kid or not a traditional AP kid or not smart enough and so when she's in like group discussion settings - especially at the beginning of the year--was much more hesitant and was much more the kid like here "will you check this for me; will you come over here and see if this is right?"- -very tentative. And she was so good, oh my gosh she was so good! At the absolute top of I would say the top $20 \%$ of my AP kids last year and not because I like her but literally if you went objectively she was in the top $20 \%$ of my kids.

Likewise as an African-American female, Alesia reveals that she is acutely aware of the role her race plays in comparison to her AP classmates. For Alesia there exists a subconscious sense of internal pressure to strive be "better than" her White classmates- 
in all aspects of schooling. Alesia explains how being African-American and female shapes her identity as a student: "I think it helps me identify myself as a good student. Someone who's working just as hard as everyone else, but there's other people, the outsiders who may think the opposite." She admits she is inspired when she sees gifted African-American students performing well in class:

In a way, it does because I see other minority students answering questions correctly and that makes me feel I can do it, but the ones that are doing the best in the class just happen to be White. And sometimes I don't see the engagement that they apparently bring to the class, sometimes [I think] they just know certain things that I don't.

Recognizing the impact race has on how she perceives herself as a student and how those around her might view her is vital the development of Alesia's academic identity.

Alesia likes school and admits having her friends in class with her makes it more enjoyable. However, she does not allow socializing with friends to distract her learning and knows when to re-engage quickly and get back on track. During class, Alesia will take on the role of a "momma duck," nudging her friends and classmates along whenever they lag behind. For Alesia, this is particularly true for classmates who bear a striking similarity to her-'non-traditional' gifted students who may be exhibiting complacency or off-task behavior.

Ms. Michaels: So a lot of times - my experience with her in the class she had last year was she was the one trying to pull other kids who didn't see themselves as traditional AP kids maybe or weren't being successful—she'd be like 'that's 
foolish" or "that's ridiculous" or "could you please stop talking" or "would you get back on track" or "wake up." So she's pulling everybody else along with her in this very sort of momma duck kind of way—like everybody get in line come on we're doing this and she was very motivational and she stayed on them.

Alesia's academic identity is comprised of physical attributes, self-identified qualities and those that others have observed in her. Her intrinsic motivation, wavering confidence, articulate communication, realistic goal planning, motherly encouragement and high expectations for academic success have become essential pieces of who she is as a student. Each of these components in addition to being African-American and gifted, make her who she is and she will somehow learn to navigate the complexities of her identity.

\section{Victoria}

Victoria's positive demeanor and stable motivation (as evidenced by...) are two features that help shape her academic identity. Although Victoria is a creative thinker, she reveals that she values structure and organization within her AP courses and prefers that her teachers progress with content in an orderly fashion. Her optimism drives her to maintain good relationships with teachers to use their expertise as a resource to grasp difficult content:

Whenever you finally get something. Every time I completely understand something it's just it's like that little kick you needed to keep on working to keep doing what you gotta do so that's always helpful. Whenever the teacher really clears it up for me that's also a really good moment cause I feel like I can trust 
that teacher and I can ask them anything and I'm the person I like to have really good relationships with my teachers so that absolutely is a bonus.

Victoria describes what it is like for when she is unable to connect with her teachers and the frustration she feels with rigorous content:

When I don't have a good relationship with my teacher. When I don't know what's going on. Times when there's so much to do I really don't know what it is I'm kind of confused on what we're talking about at the moment, whenever I'm really deep in confusion that's just my huge biggest thing. And I can't...I ask a teacher and they just tell me like 'I don't know what to tell you, what do you think the answer is?' Well then I'm just even deeper in confusion 'cause I think the answer is all the answers, I don't know what the answer is. So then I'm lost every time I'm confused and then I don't have a good relationship with my teacher. That don't help at all either and then I've got none of my peers to help me out and I'm just confused.

As a gifted student, Victoria learned to use available resources as a problem-solving skill and her relationships with teachers is a tool she uses to access a valuable resource. When her teacher-resource is unavailable because of lack of rapport, Victoria becomes aggravated as her search for deeper learning is hindered temporarily-

until she discovers alternative resources that can help.

Victoria reports that her identity is not influenced adversely by her peer groups, and that instead, her motivation improves whether her peers are being negative or positive. She had this to say about her AP peers in class: 
When I see that kid that knows all the answers I want to be that kid too. So that really I guess motivates me to be work-oriented um whenever we do group work and some people aren't participating that's when teamwork and communicating kicks in and we just got to get it done.

Mr. Simmons observes Victoria's interactions with AP peers and perceives their influence on her as positive, stating:

I don't know.... I think she knows the "top dogs" in our school and she makes strides to get to them, to get to those places and I think that she wants to be one of those "top dogs" so she pushes herself to get to those places - but other than that I'm not quite sure.

Victoria stated that her traditional peers also motivate her to work harder, despite their occasional negative comments:

Victoria: Yeah. They make me feel like I'm really working hard that I'm staying on my stuff. And they make me want to work harder and motivate them so we can all be on our stuff and we can all get this work done together! So they make want to be somebody that I can look up to I guess if I were to look at me-am I doing what I need to do.

Interviewer: What about the negative ones? The ones that say "why are you doing this; why are you taking these classes?" Do they influence what you think about yourself as a student at all?

Victoria: Um sometimes yeah. They make me feel like I'm unnecessary, I'm doing too much at this moment that I don't have to do like that's always what I 
get, like you really don't have be doing all that. 'Cause it's like I really don't have to be taking that class but I know that it's good for me.

Another important feature of Victoria's identity is her candidness among peers when it comes to sociocultural issues. Ava described observations of her friend's classroom conversations with gifted peers, pointing out her strong defense of AfricanAmerican culture when an issue arises:

....so like sometimes they would say things that she doesn't necessarily believe, kind of offensive towards Black people, not meaning it, but kinda like she's super pro-black — so whenever anyone says something she kinda like hops on them (snaps fingers). But it's nothing negative, she just wants them to see it from a different perspective.

For Victoria, the development of an academic identity is backed by strong intrinsic motivation and does not appear to be negatively affected by peers. Her interactions with peers seem to intensify her motivation to improve herself or allow her to turn a misguided comment from a classmate, into a teachable moment about culture.

Finally, her positive attitude and outgoing personality allows her to be able build relationships with teachers and other adults.

\section{Cross-Case Analysis}

I conducted a cross-case analysis in order to compare and contrast study findings across all participants: student-informants, peers, and trusted adults. Cross-case analysis evaluates each case separately and then compares data across all cases. Baxter and Jack (2008) suggested that cross-case analysis is beneficial process for researchers to engage 
in, as it enhances the case and increases credibility. The theoretical frameworks of selfefficacy and academic identity, along peer group relationships, are each designated as broad categories. Subcategories and themes resulting from first and second cycle coding of interview transcripts and field notes are assigned to each category as determined by their relevance.

Cross-case analysis results are organized into three tables with theoretical frameworks outlining the first column, followed by codes as subheadings in the second column. Table 7 highlights cross-case analysis results derived from participant responses to questions about peer group relationships and interactions with gifted and traditional peer groups, as well as how each interaction may have influenced informant self-efficacy or academic identity. Each feature is coded with symbol representing how each informant responded to or acknowledged the peer relationship statement. Table 7 and Table 8 provide key evidence that demonstrates the connection between informant responses and the frameworks of the study (e.g., self-efficacy, academic identity, peer relationships). 
Table 7

Cross-Case Analysis of Participant Findings-Peer Relationships

\begin{tabular}{|c|c|c|c|c|c|c|c|c|c|c|}
\hline 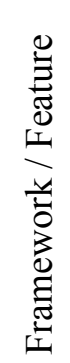 & $\begin{array}{l}\text { Cyclic Codes } \\
\text { (First and Second) }\end{array}$ & 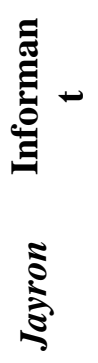 & $\frac{\mathbb{Z}}{\mathbb{Z}}$ & 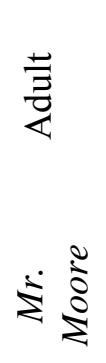 & 离 & 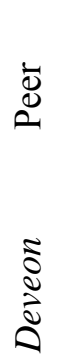 & 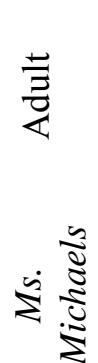 & 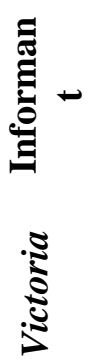 & 㐫 & 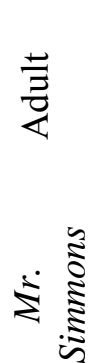 \\
\hline \multirow{9}{*}{ 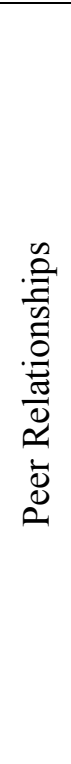 } & $\begin{array}{l}\text { Peer Group Similar to } \\
\text { Informant }\end{array}$ & $\mathbf{O}$ & & $\mathrm{O}$ & $\mathbf{X}$ & & $\mathrm{X}$ & $\mathbf{X}$ & $X$ & \\
\hline & $\begin{array}{l}\text { Past Peer Group Mix of } \\
\text { Traditional and Gifted Peers }\end{array}$ & $\mathbf{X}$ & & & $\mathbf{X}$ & & & $\mathbf{X}$ & $X$ & \\
\hline & $\begin{array}{l}\text { Current Majority of Peers are } \\
\text { Gifted }\end{array}$ & $\mathbf{O}$ & & & $\mathbf{X}$ & & $X$ & $\mathbf{X}$ & $X$ & \\
\hline & $\begin{array}{l}\text { Majority of Peers are } \\
\text { African-American }\end{array}$ & $\mathbf{X}$ & & & $\mathbf{X}$ & & & $\mathbf{X}$ & $X$ & \\
\hline & $\begin{array}{l}\text { Gifted Peer Comments on } \\
\text { AP Choice }\end{array}$ & + & & & -- & & & & + & \\
\hline & $\begin{array}{l}\text { Traditional Peer Comments } \\
\text { on AP Choice }\end{array}$ & -- & & & + & & & $-1+$ & -- & \\
\hline & $\begin{array}{l}\text { Perception of Gifted Peer } \\
\text { Academic Performance }\end{array}$ & + & & + & $-/+$ & & & $-1+$ & & \\
\hline & Gifted Peer Interactions & + & + & + & + & + & + & -- & + & + \\
\hline & $\begin{array}{l}\text { Traditional Peer Interactions } \\
\text { following AP Enrollment }\end{array}$ & -- & & & + & + & & $-1+$ & -- & + \\
\hline $\begin{array}{l}\text { Not } \\
\text { part } \\
\text { negc } \\
\text { prol }\end{array}$ & $\begin{array}{l}X \text { denotes code confirmed by } \\
\text { pant; }(+) \text { denotes a positive r } \\
\text { ve response received from stuc } \\
\text { e a response. }\end{array}$ & onse & & $t ; O d c$ & $\begin{array}{l}\text { enotes } \\
\text { i study } \\
\text { k indic }\end{array}$ & & ot conj & irmed & & \\
\hline
\end{tabular}

Informants agreed on several factors related to peer relationships, such as majority of their friends are gifted African-Americans. Informants also acknowledge a shift in composition within the peer group — from mixed achievement level to predominatelygifted peers.

Table 8 organizes participant responses to questions on self-efficacy and academic identity. Each feature is coded with symbol representing how each informant 
responded to or acknowledged the statement. For attributes such as confidence and motivation, responses were coded as low/average/high or low/present, respectively. Other items in Table 8, represented by symbols, are defined at the bottom of the table and reflect the type of response given by each informant.

\section{Table 8}

Cross-Case Analysis of Participant Findings-Efficacy and Academic Identity

\begin{tabular}{|c|c|c|c|c|c|c|c|c|c|c|}
\hline \multirow{2}{*}{ 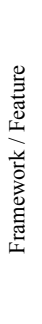 } & \multirow[b]{2}{*}{$\begin{array}{c}\text { Cyclic Codes } \\
\text { (First and Second) }\end{array}$} & : & $\begin{array}{l}\vec{\Xi} \\
\stackrel{0}{0}\end{array}$ & $\frac{\vec{Z}}{\frac{7}{4}}$ & 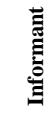 & 岕 & 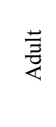 & $\stackrel{\vec{\Xi}}{\stackrel{\Xi}{\Xi}}$ & $\begin{array}{l}\overline{\mathrm{d}} \\
\stackrel{0}{0}\end{array}$ & $\frac{\overrightarrow{7}}{\frac{7}{2}}$ \\
\hline & & $\stackrel{\overline{5}}{\overline{2}}$ & $\underset{\mathfrak{Z}}{\mathfrak{Z}}$ & 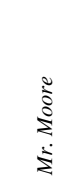 & 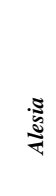 & $\begin{array}{c}\bar{\Xi} \\
\stackrel{\Xi}{\Xi}\end{array}$ & $\begin{array}{l}\frac{\tilde{\Xi}}{\Xi} \\
\stackrel{\Xi}{\Xi} \\
\dot{\Xi} \\
\dot{\Sigma}\end{array}$ & 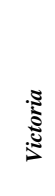 & $\stackrel{\square}{\dot{\psi}}$ & 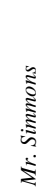 \\
\hline \multirow{8}{*}{ 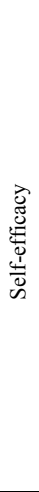 } & Positive Self-belief statements & $\mathbf{O}$ & $\mathrm{X}$ & & $\mathbf{X}$ & $\mathrm{X}$ & $\mathrm{X}$ & $\mathbf{X}$ & $\mathrm{X}$ & $\mathrm{X}$ \\
\hline & Negative Self-belief statements & $\mathbf{X}$ & $\mathrm{X}$ & $\mathrm{X}$ & $\mathbf{X}$ & & $\mathrm{X}$ & & & \\
\hline & $\begin{array}{l}\text { Self-confidence in completing rigorous tasks } \\
(\mathrm{L}=\text { low } ; \mathrm{A}=\text { average } \mathrm{H}=\text { high })\end{array}$ & $\mathbf{H}$ & $\mathrm{n} / \mathrm{a}$ & $\mathrm{n} / \mathrm{a}$ & $\mathbf{H}$ & & & $\mathbf{H}$ & & \\
\hline & $\begin{array}{l}\text { Self- confidence in passing AP exam } \\
(\mathrm{L}=\text { low } ; \mathrm{A}=\text { average; } \mathrm{H}=\text { high })\end{array}$ & $\mathbf{L}$ & $\mathrm{n} / \mathrm{a}$ & $\mathrm{n} / \mathrm{a}$ & $\mathbf{L}$ & & $\mathrm{L}$ & $\mathbf{L}$ & & \\
\hline & $\begin{array}{l}\text { Self-confidence in potential to pass AP } \\
\text { course }\end{array}$ & $\mathbf{A}$ & $\mathrm{n} / \mathrm{a}$ & $\mathrm{n} / \mathrm{a}$ & $\mathbf{H}$ & & & $\mathbf{A}$ & & \\
\hline & $(\mathrm{L}=$ low $; \mathrm{A}=$ average $; \mathrm{H}=$ high $)$ & & & & & & & & & \\
\hline & $\begin{array}{l}\text { Self-Motivation } \\
(\mathrm{L}=\text { low; } \mathrm{P}=\text { present })\end{array}$ & $\mathbf{L}$ & L (obsv) & $\begin{array}{c}\mathrm{L} \\
\text { (obsv) }\end{array}$ & $\mathbf{P}$ & $P$ & $\mathrm{P}$ & $\mathbf{P}$ & $\mathrm{P}$ & $\mathrm{P}$ \\
\hline & Acknowledged Peer Influence on Efficacy & & $\mathrm{X}$ & $\mathrm{X}$ & $\mathbf{X}$ & & $\mathrm{X}$ & & $\mathrm{X}$ & \\
\hline \multirow{7}{*}{ 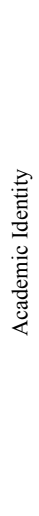 } & Acknowledged race as part of identity & $\mathbf{X}$ & $\mathrm{O}$ & $\mathrm{X}$ & $\mathbf{X}$ & & & $\mathbf{X}$ & & \\
\hline & $\begin{array}{l}\text { Experience with racial identity in gifted } \\
\text { courses }\end{array}$ & -- & & & -- & & & & & \\
\hline & Acknowledged giftedness as part of identity & + & & & $\mathbf{X}$ & & & & & \\
\hline & $\begin{array}{l}\text { Experiences with giftedness as part of } \\
\text { identity }\end{array}$ & -- & $-1+$ & & & & & & & \\
\hline & Identified Gifted Student Qualities & $\mathbf{O}$ & $\mathrm{X}$ & $\mathrm{O}$ & $\mathbf{X}$ & $\mathrm{X}$ & $\mathrm{X}$ & $\mathbf{X}$ & $\mathrm{X}$ & $\mathrm{X}$ \\
\hline & $\begin{array}{l}\text { Personal Features } \\
\text { ( } \mathrm{E}=\text { external motivation; } \mathrm{P}=\text { parental } \\
\text { pressure; } \mathrm{I}=\mathrm{I} \text { can } / \text { will })\end{array}$ & $\mathbf{E}$ & E & $\mathrm{E}$ & $\mathbf{P}$ & & $\mathrm{P}$ & I & I & I \\
\hline & $\begin{array}{l}\text { Peer Influenced Factors } \\
(\mathrm{Cd}=\text { co-dependency; } \mathrm{Co}=\text { comparison; } \mathrm{M}= \\
\text { motivation })\end{array}$ & Cd & & $\mathrm{Cd}$ & Co & & Co & $\mathbf{M}$ & M & M \\
\hline
\end{tabular}

Note: $X$ denotes code confirmed by study participant; $O$ denotes code not confirmed by study participant; $(+)$ denotes a positive response received from study participant; (-) denotes a negative response received from study participant; a blank indicates study participant did not provide a response. 
Student-informants varied in most of their academic identity features, yet all acknowledged being African-American as part of their identity. Motivation was an efficacious feature present in each informant and acknowledged by both peers and adults. Other aspects of efficacy were varied in identification by the informants themselves and also in confirmation from peers and trusted adults.

\section{Summary}

In this chapter, I presented the data collected to answer the research questions posed for this study. The findings are drawn from data provided by the nine study informants from North High School, including six students (three studentinformants and three of their peers) along with three trusted adults. The data collected from all informants in this study provided insight into the perspectives of AfricanAmerican students in Advanced Placement courses—in particular, how they and others around them perceive peers (AP and traditional peers) as influencing their academic trajectory. The primary informants were the three African-American students at North High School who enrolled in AP classes, seeking to follow a rigorous pathway that would prepare them for successful post-secondary matriculation, but it did not come without its challenges. As expected upon transition into gifted programming, students would experience higher academic expectations, difficult coursework and increased accountability, yet for these students, the most arduous tasks were not exclusively academic. For these students, self-identifying as "smart kids" also was accompanied by internal struggles associated with questions of academic identity and self-confidence, as well as learning how to navigate new social stereotypes and peer group nuances. 
The relationships and interactions with peers, at least to some degree, seemed to influence the academic efficacy of student-informants. Alesia and Victoria acknowledged their perceptions of gifted peers as impactful to the extent that it created pressure for them to prove themselves academically. Other informants (interviewed peers and trusted role models) for both young women agree that interactions with gifted peers, whether positive or negative, only fueled their resiliency and intrinsic motivation for high academic achievement. In contrast, Jayron admittedly does not harbor strong self-efficacy and does not perceive his peers as having a direct influence on his academic beliefs. However, his peer and adult informants agreed that Jayron is heavily dependent on his gifted peers for academic support and that much of his potential remains unrealized because of his low confidence.

Each student informant shared their experience as self-identified gifted student and their perspective on how peers influence their academic beliefs and thoughts about who they are as students. Gifted student-informants identified peers and adults to share further insights on the gifted experience. I divided this chapter into sections designed to introduce the reader to each informant first, how they came to enroll in AP courses, perspectives on peer interactions, efficacy and formation of academic identity. Within each category, I presented the findings for each participant separately. Analysis of state and district documents and student files provided additional information about school demographics, informant grade level, gifted trajectory, academic achievement and assessment data. First cycle structural coding and second cycle data theming were used to develop categories and show themes across all participants. Efficacy ratings are presented in Table 4, while categories and codes derived from participant responses were organized 
by peer group relationships, efficacy and academic identity (See Tables 5 and 6 , respectively). Chapter V discusses key findings and implications for policy, practice and future research. 


\section{CHAPTER V: DISCUSSION}

Empirical research on students of color who self-identify as gifted by electing to enroll in AP courses through open enrollment processes is, at best limited if not nonexistent (Awaya, 2001; Winebrenner, 2006). The aim of this study was to provide a response to the lack of research and to provide insight into the perspective of this group of unique students. Through this study, I sought to explore how African-American high school students who voluntarily enrolled in Advanced Placement courses (e.g., open enrollment) perceived their peers as influencing their self-efficacy and their academic identity. To address this, I posed two research questions: (1) What are the perceived influences of peers on the development of self-efficacy and academic identity of AfricanAmerican students enrolled in Advanced Placement courses? (2) How do AfricanAmerican students in Advance Placement courses perceive interactions with peers and cultural ideas towards achievement, as influencing their overall academic experiences in Advance Placement courses?

My study findings provide practitioners insight into gifted students' perspective which may be valuable when making data-based decisions about ways to improve AP program recruitment and retention. The data presented are insightful for practitioners and administrators at all levels should consider experiences by informants as insightful 
evidence for academic and socioemotional supports that promote positive expectations and identity development.

This data is particularly useful for building-level administrators seeking to modify admission policies and implement support programs to ensure successful matriculation of African-American students in gifted courses. The remaining sections of this chapter include a discussion of the key findings from this study followed by implications for practice, policy and future research.

\section{Peer Group Relationships}

The cross-case analysis revealed several answers to my both research questions regarding perceived influences of peers on efficacy and identity development for gifted students of color. Student-informants shared several factors in common with each other regarding peer group composition (refer to Table 7 and Table 8). The commonalities among student-informant peer groups aligns with the idea of model similarity wherein adolescents become more similar to one another the more they interact with each other (Hymel, Comfort, Schonert-Recihl \& McDougall, 1996; Schunk \& Pajares, 2001. Research suggests that model similarity significantly influences a student's perception of his or her own academic abilities and ultimately their achievement (Bandura 1986; Schunk 1987; Schunk \& Pajares, 2001). Each participant reported having primary peer groups whose personalities and academic values were similar to their own. Jayron and Victoria characterized their circle of friends as "positive" with a driven work ethic, while Alesia compares her friends to her own likeness, admitting they have a lot in common. Likewise, all agreed they would not enjoy school without having their friends in class. 
When asked about the race/ethnicity of their peer groups, all participants responded that the majority were African-American. In fact, the peers they recommended as peer informants were all African-American. Jayron and Alesia could not clearly explain why most of their friends happened to be African-American; however, Victoria attributed this dynamic to the difficulties she faced making friends in a predominately White elementary school. For students of color, association with homogenous peer groups signifies a strong awareness of cultural norms and quite simply, is more comfortable for them. Nevertheless, gifted African-American students often feel compelled to disassociate from their gifted identities in response to peer pressure to comply with culture norms - to be smart and do your work is to "act White" (Ford \& Grantham, 2003; Ford \& Thomas, 1997; Fordham \& Ogbu, 1986; Ogbu, 1991). Two of the student-informants said they felt pressured to deny their "giftedness" to their peer group, but reported having moments in which they felt less intelligent than White gifted classmates. Victoria reported experiences in which her African-American peers criticized her for her choice to take AP classes, saying that she was working too hard and did not "hang out" to the extent that she had prior to enrolling in AP courses. Despite this, Victoria's strength of identity allowed her to remain focused on her goals.

Two out of three student-informants stated that the majority of their friends were gifted students and they shared some AP classes with those friends. Alesia admitted she did not realize that most of her friends were AP students until it was brought to her attention during this interview. Victoria echoed this statement, not noticing that she had drifted away from her non-AP classmates. Nevertheless, both young ladies acknowledged they still associate with non-AP friends occasionally and attribute this peer group shift to 
their decision to enroll in AP courses-- subsequently having fewer encounters with former traditional peers. Jayron reported that his friends were an even mix of gifted and traditional friends from his honors (comprehensive) schedule the first two years and the AP classes added during the last two years.

During the interview, questions related to peer relationships sought to illicit information about two distinct groups: AP and traditional (non-AP) peers. Studentinformants noted distinct differences between how they communicated with these two peers groups. Alesia and Victoria reported that their AP peers were easier to talk to because they could relate to what they were experiencing. They reported sharing similar post-secondary goals and general conversations that were more interesting. Both women stated they had both positive and negative conversations about their AP choice with traditional peers - negative conversations left them with brief second thoughts. Jayron did not refer to differences between how he communicates with either peer group. Alesia and Jayron admitted that sometimes during AP class discussions they begin to doubt themselves when they cannot answer a question and feel like they do not belong in this class. Victoria noted that during discussions she channels her lack of knowledge into competitive motivation to learn what her classmates already know. Scholars contend that the level of value students place on peer relationships makes peer group perceptions an influential factor on academic beliefs, confidence and achievement (Ryan, 2000; Wentzel, 1991). In my study, Mr. Moore discussed Jayron's dependency on peer responses before answering questions aloud in class. Likewise, Alesia reported moments of academic inferiority triggered by her inability to keep up with the rapid responses of her classmates. Thus, an intrinsic process occurs as students consider peer opinions 
subconsciously when struggling with difficult tasks and intentionally when making social and academic decisions.

\section{Self-Efficacy}

Self-efficacy is a component of social cognitive theory, which is driven primarily by the process of motivation (Bandura, 1982; 1986; 1993). Efficacy describes a person's belief in one's own ability to complete a task to achieve an expected outcome. The student-informants responded to a series of questions wherein they rated their efficacy pertaining to AP tasks (See Table 3). Informant data on self-efficacy provided answers to both research questions. Surprisingly, all informants rated themselves similarly (average 6.5) when it came to belief in their potential to succeed in AP courses. Despite moments of wavering confidence, they were confident in their ability to perform well academically. Likewise, each participant was very confident (average 7.6) in their ability to complete rigorous assignments despite complaining about self-induced stress from the amount of course work. Teachers/mentors, Ms. Michaels and Mr. Moore both spoke about supporting gifted students when they felt overwhelmed and discouraged — helping to build resilience. Each student-informant reported very low confidence in their ability to pass an AP examination (average 2) and receive college credit. For Jayron and Alesia, this self-efficacy rating was expected, given that they had taken at least one AP exam, but had not received college credit as evidenced by a score of 3 or higher. Victoria took two AP examinations and received college credit for both of them, yet she rated her efficacy as "fairly low". She explained that her confidence level was dependent upon the course subject area and how well she felt the teacher covered the material. 
Individually, each student-informant varied with regards to the factors that comprised their efficacy beliefs, in particular when it came to the source of their academic motivation. Bandura (1986) acknowledged that motivation was a key factor in determining the level of self-efficacy. Jayron lacked the intrinsic self-motivation to drive his efficacy and clearly depended on encouragement and support from those around him to boost his confidence and work ethic—co-dependency. Alesia's efficacy stems from motivation that was rooted in her parents' expectations for her and the resulting pressure she places on herself to achieve greatness. She acknowledged that comparing herself to classmates weakens her confidence and causes her to doubt her giftedness. Victoria stabilized her motivation through scholarly poise and determination to learn which kept her focused. She indicated that she believes her peers boost her motivation, making her work harder and persevere. Pajares (1996) contended that differences in self-efficacy levels are expected, suggesting that cognitive processes coupled with intrinsic motivation makes self-efficacy a "task-and-situation-specific" (p.546) factor and as such it varies across individuals. Although they share giftedness and race in common, the way in which they cultivate beliefs about themselves and their academic abilities is very different. Further, how they perceive peers as influencing these beliefs strikingly different despite similarities among peer groups.

\section{Academic Identity}

Within this study, efficacy beliefs overlapped with academic identity, as evidenced by participants describing how they viewed themselves as students while simultaneously speaking about their level of confidence in their academic abilities. Race stood out as a prominent feature of academic identity. The semi-structured style of the 
interview protocol helped to address the academic identity components of my research questions and sparked reoccurring conversations about race. Each student-informant acknowledged that being African-American played a role in how he or she perceived themselves as AP students. Jayron discussed how race and gender made him feel uncomfortable in gifted classrooms because he believed peers and adults were acutely aware of his unique presence in the room. Alesia reported that being an AfricanAmerican female meant she essentially had to demonstrate she was as smart as her classmates and work harder to measure up. Victoria revealed that her racial identity pushed her to be more vocal on equity issues, particularly during AP class discussions when the topic of race came up.

Self-motivation was a fundamental feature of each student informant's academic identity. Self-motivation for Jayron was the lowest among the three student-informants and was dependent upon the encouragement of the peers and adults around him. Alesia's motivation was intrinsic and driven by high expectations from her parents. Victoria's scholarly drive fueled her motivation to perform well academically.

\section{Study Implications}

Having summarized the findings of my study, I now turn my attention to the implications that this study may have for policy, practice, and future research. First, I discuss implications for policy, urging education lawmakers to implement open enrollment protocols, but with some suggested modifications to improve outcomes for African-American gifted students. I then turn to the implications for practice; specifically that of building-level stakeholders, such as program coordinators, principals, assistant principals, school counselors, and teachers all working collaboratively to improve 
educational experiences and academic outcomes for Advance Placement students, in particular those who voluntarily enrolled through open access protocol. Finally, I suggest directions for future research with gifted African-American students including the effectiveness of open enrollment and support programs on improving achievement, sociocultural dynamics of peer relationships and academic achievement, as well as a shift in higher education protocol regarding gifted coursework.

\section{Implications for Policy}

The results of my study highlight several factors that educational policymakers and stakeholders at the state and district levels should consider with regard to enrolling high school students of color into gifted education programs. First, recouping current AP data for my study proved to be a challenging task, as the most current data available was from the 2013-14 academic year. This suggests the need for a change in data tracking and management policies at district level to ensure accurate records of AP enrollment numbers and AP data are available to schools. In this large school district, lags in data tracking are problematic and impede purposeful planning efforts at the school-level for administrators seeking to improve gifted education or develop supplemental programs to support students like Jayron, Alesia and Victoria.

This study contributes to a growing body of research suggesting that open enrollment and self-nominating (Coleman, Gallagher \& Foster, 1994) admission processes are viable protocols at the school-level for identifying students of color for gifted courses. Although open access challenges traditional enrollment practices as a predictor of AP success, the inherent tracking methods used by many schools to group minority students into low-level courses subsequently limits their exposure to rigor and 
the critical skills needed to meet elevated achievement expectations (Baldwin, 2004; Edward \& Duggan, 2012; Ford et al., 2001; Ford \& Grantham, 2003; Fordham \& Ogbu, 1986; Fordham \& Thomas, 1997; Grantham \& Ford, 2003; Klopfenstein, 2004; Ndura, Robinson \& Ochs, 2003; Siegle, 2001; Solozano, 2004). In this way, voluntary enrollment may unintentionally perpetuate the problem of minority underachievement by providing access to students who are unprepared for rigorous coursework. Despite this, the benefits of providing AP access to nontraditional students are critically important for post-secondary goals and it is the responsibility of school-level administrators to ensure school policy reflects efforts to recruit and retain gifted students of color.

Federally funded efforts aimed at closing the achievement gap sought to increase access for marginalized students (e.g., low income, African-Americans, Latino/a) to Advanced Placement (AP) courses without standardized testing, as well as supplementing the cost of cumulative exams. These federal efforts helped to fuel initiatives such as AdvanceState, which individual schools in this district (e.g., North High) elected to adopt as part of their gifted admission protocol. Each student and peer-informant in this study gained access to AP classes through AdvanceState open enrollment protocol. As a result they provided the opportunity to participate in rigorous coursework and learn problemsolving skills deemed necessary for post-secondary progression. Although their presence in AP courses helped to increase diversity at North High, at times student-informants were made acutely aware of their unique presence. For example, Jayron expressed his explicit awareness and the acknowledgement of others around him, including the principal, that he was the only African-American male in an AP class. Alesia recalled moments where she felt less intelligent than her gifted classmates when she could not 
answer a question. Modest increases in diversity are admirable, however occurrences such as these warrant the need for school-level administrators to improve recruitment and retention efforts for gifted African-American students. Recruitment policy should incorporate open enrollment protocol as part of the gifted program admission process, while retention efforts focus on creating holistic support programs.

\section{Implications for Practice}

There were modest increases in nontraditional enrollment at North High and other school across the country (Ford, 1998; Ford \& Thomas, 1997; Grantham \& Ford, 2003; Kao \& Thompson, 2003; Klopfenstein, 2004; Kyburg et al., 2007; Ndura, Robinson \& Ochs, 2003). However, simply enrolling students was not enough to ensure successful matriculation. For gifted African-American students, the self-nomination into AP courses present challenges beyond rigorous the coursework for which they may be inadequately prepared, as they also must cope with identity development issues and related emotional stressors resulting from peer judgements about 'acting White' (Ford, 1998; Ford \& Thomas, 1997; Fordham \& Ogbu, 1986; Rodgers, 2008). It is the collective responsibility of administrators, counselors, program coordinators, and teachers to support the successful matriculation of gifted minorities. In her analysis of sociocultural issues surrounding the recruitment and retention of gifted minorities, Ford (1998) insists that educators must do more to support students: "the quality of students' educational experiences must be examined prior to identification and placement so that students will be successful in the gifted education program" (p. 11). The student voices featured in this study highlight the need for targeted resources designed to help feel better prepared to sit for AP exams. Student-informants reported they feel unprepared for Advance Placement 
exams (Table 5) and all suggested that an African-American support group to study with would be beneficial. Students need to learn how to analyze information, think critically to solve problems and some may require reinforcement of fundamental concepts that will allow them to attack rigorous tasks head-on. Equipping students with academic tools will address the academic deficiency piece, but students must still find ways to contend with contentious attitudes from peers. Informants acknowledged shifts in peer groups where they were finding themselves wanting to associate more with African-American peers even though at times, these same peers were the ones questioning their gifted choice. These students must find ways to navigate tumultuous relationships, focus on academic coursework, discover their identity, all while remaining committed to the cultural norms of the racial group - a socioemotional roller coaster.

In her analysis of gifted African-American students, Rodgers (2008) argued that sociocultural constructs and racial identity peer pressure amplifies emotional sensitivity to judgement. Further, when gifted minorities are subject to negative comments, ostracizing and even isolation by individuals they value, it leads to underperformance in the classroom (Rodgers, 2008). For example, in this study each student informant spoke of a peer(s) who criticized their decision to enroll in AP classes. Alesia spoke of peers who suggested she withdraw from gifted courses because of the amount and rigorous intensity of assignments and although she was able to dismiss the negativity and push forward, for those who lack resilience educators must do more to encourage them to prevent possible lags in achievement. Student-informants also agreed that the presence of an after-school group exclusively for AP students of color would be beneficial to assist them with test preparation, as well as provide a social outlet to share experiences. Gifted 
program coordinators, in collaboration with school counselors and teachers can create supplemental support services for gifted minorities to ensure successful matriculation. Stallworth and Thomas (2017) suggest the use of support groups and mentors to help diminish sensitivity among gifted minorities. As part of their intervention programs, AVID (detailed in Chapter II) and Project Promise ${ }^{6}$ foster positive interpersonal relationships by encouraging teacher-student and student-student interactions, along with selecting high-achieving African-American and Latino/a students and pairing them up with participants to act as mentors. School administrators, coordinators, counselors and teachers should consider both the positive and negative experiences of peer group interactions presented in this study and consider including mentors in the organization of their own gifted support programs. Mentors can help students navigate the sociocultural pressures that if not addressed, could result in a growing trend of academically capable students of color who remain stagnant in unrealized potential.

Gifted African-Americans who voluntarily participate in gifted courses and then underperform academically, challenge the purposeful intent of open enrollment inevitably contribute add to the achievement gap. In addition to sociocultural resources, gifted students who struggle with rigorous coursework would benefit from academic resources that include targeted interventions to address deficiencies. In order to sustain their comprehensive support programs, it is imperative that building-level administrators seek out ways to increase opportunities for gifted African-American students to find success. Existing support programs such as AVID and EXCITE are excellent templates for district

\footnotetext{
${ }^{6}$ University for Young People's Project Promise is a tuition-free summer enrichment program located on a college campus designed to promote academic potential in gifted students from $4^{\text {th }}$ to $12^{\text {th }}$ grades (Kaul, Johnsen, Witte \& Saxon, 2015).
} 
and school-level stakeholders to develop their own comprehensive resources to address both academic and nonacademic needs. AVID provides academic and social resources to motivate minority students to challenge their achievement potential by taking collegepreparatory courses in high school. Project EXCITE is an intervention program created to increase academic achievement among gifted students of color. The goal of EXCITE was to improve students' chances of being accepted into gifted education programs by increasing their academic skills and proficiency with rigorous tasks (Olszewski-Kubilius, 2006; Olszewski-Kubilius et al., 2004). The EXCITE program is primarily focused on recruiting students into Science Technology Engineering and Math (STEM) careers programs, but its designers incorporate intentional social opportunities with mentors and encourage teachers to build relationships with students in order to create buy-in for a culture of high expectations.

Programs with similar sociocultural frameworks to AVID, Project EXCITE and Project Promise should be mandatory for schools and districts seeking to adopt open enrollment policies for AP admissions in order to ensure students are truly benefitting from 'getting in'-earning college credit on cumulative exams and acquiring college preparatory skills. To be most effective, these programs should be holistically designed to effectively address the needs of the whole child. This requires involvement from all stakeholders including administrators, coordinators, counselors, teachers and students-input from each role group adds valuable perspective and creates buy-in, providing a sense of program ownership (Kent, 2004). Gifted student voice is a valuable asset to program planning and the educational experiences and self-identified needs of these 
students can help determine specific support program components (e.g., academic skills, socioemotional support, mentoring).

Administrators must ensure that gifted teachers are fully prepared to support the diverse needs of nontraditional gifted students, in particular gifted African-American students. In addition to content-based AP training and certification, administrators must make culturally relevant professional development available to enhance pedagogy and encourage sensitivity towards sociocultural issues (e.g., Jayron's encounter with his principal). Current reports indicate that the district site for this study is making efforts to improve the quality of culturally relevant practices in its schools by offering professional development opportunities to all staff throughout the school year. It is important to note the district's use of research-based culturally relevant professional practices and curriculum, which add both value and relevance once implemented. Teachers and administrators must work collaboratively to implement culturally relevant practices with fidelity and ensure ample opportunities exist for continuous reflection and growth to improve pedagogical competency. Likewise, higher education institutions play a fundamental role in preparing future practitioners for diverse gifted classrooms. Administrators of educator preparation programs are tasked with ensuring graduates are adequately prepared to work with diverse K-12 gifted students. Pre-service educators should be made aware of pervasive academic issues such as low-level tracking, as well as the sociocultural issues faced by gifted African-American students and to how best to support them.

Ideally, once implemented at the school-level comprehensive gifted support programs would be funded at district or state level as an addendum to the gifted 
education budget. As previously discussed, it is counterproductive to increase Advanced Placement (AP) access for students of color without creating specialized systems to ensure their success. Taking shortcuts will continue to exacerbate minority underachievement issues such as Jayron's codependency and low confidence about AP exams reported by all student informants. The real experiences shared by all informants highlight neglect on the part of educators to consider the ramifications that open enrollment could have on achievement, efficacy and identity development. Thus, in order to be successful at recruiting, retaining and supporting gifted African-American students, open enrollment programs will require commitment, collaboration, expertise and thoughtfulness from all stakeholders.

\section{Implications for Future Research}

My study explored the educational and sociocultural experiences of gifted African-American students who elected to enroll in AP courses through an open access program at their school. Despite the generalizability limitations of this study that were previously outlined, the data presented here raises additional questions about the contextual factors of this study and their effectiveness in addressing issues surrounding academic success for gifted students of color. Future research that expand upon the limitations of this study, would prove beneficial for stakeholders struggling to address achievement gap, as well as those seeking to support underachieving gifted minorities.

One suggestion for future research would be to pursue quantitative research designs that determine the success rate of state-level open enrollment programs at increasing access for traditionally underserved populations (e.g., low-income, minority students). One essential question: Are nontraditional students attending schools where 
they have voluntarily enrolled in gifted courses, more academically successful compared to gifted students whose school uses traditional measures of eligibility? A noteworthy challenge for such a study would be the researcher's accessibility to a large enough sample size of open-access gifted minorities in order to establish a credible comparison to traditionally enrolled gifted minorities. Although open enrollment or self-nomination initiatives have expanded over the years (Ford, 1998), the number of districts or individual schools utilizing its protocol may be difficult to ascertain. This will pose a challenge for researchers attempting to compare two distinct groups of students within demographically similar schools, however the data would be important to understanding scope and effectiveness of self-nominating protocols. Open access programs sought to increase access to gifted education and in doing so, narrow the achievement gap-a goal realized according to educators at North High School. Yet, despite slight increases in diversity in gifted classrooms, it remains undetermined if continuing to fund these programs is truly adding value as a worthwhile effort aimed at improving minority achievement.

Another suggested research topic would be to determine if a correlation exists between gifted student achievement, as measured by standardized assessments such as AP tests or college-entrance exams, and the sociocultural dynamics of peer group relationships, particularly for African-American students in gifted education. Perhaps such a study would consider if or to what extent the type of peer interactions, positive, negative or neutral, are impactful to gifted student efficacy and academic performance. Issues for researchers would include in what context to observe peer interactions, deciding on a reliable instrument for determining the neutrality (or not) of peer 
interactions as interpreted by gifted students and presenting the data in a credible manner. Resulting data would provide stakeholder insight into the development of supplemental programs with embedded sociocultural components addressing the inherent needs of the students served.

Future researchers could explore the effects of intervention, enrichment, and support programs on academic achievement gifted students of color. Extant literature suggests that large-scale programs like AVID, Project EXCITE and Project Promise result in improved motivation, academic skill and efficacy for participating students (AVID, 2017; Olszewski-Kubilius, 2006; Olszewski-Kubilius et al., 2004; Kabul et al., 2015). Research to highlight effectiveness for local programs developed at the district or school-level may provide useful data for those working with smaller student populations or with limited funding sources. An essential component of this research would be supplemental programs linked directly to AP open enrollment initiatives as case studies to compare exam scores for low-income and gifted students of color prior to and following the incorporation of support programs to determine if there is any improvement. Ideally, study data would indicate more students passing AP exams to receive college credit, which would lead to the spread of open access protocol. Moreover, if more schools utilize open enrollment protocol then the need for effective support programs increases and stakeholder efforts would be focused on improving academic outcomes, gifted program retention rates and overall educational experiences for nontraditional students. This research would add to small batch of existing literature on open access programs, as well as contribute to the research base on issues concerning gifted students of color. 
Lastly, recent media reports indicate a rising trend among post-secondary institutions that are no longer accepting AP and dual credit coursework from incoming students (Ben-Achour, 2013). For example, institutions like Dartmouth College which has a competitive admission process, elected not to accept earned college credit citing that college preparatory courses are not rigorous enough at the high school level (Chappell, 2013) therefore students arrive inadequately prepared for college coursework. Researchers should be prepared to investigate the effects of this trend should it continue, as it could interfere with efforts like open enrollment designed to increase access to gifted education and improve college readiness among students of color.

\section{Conclusion}

The review of literature, research, and results of my study emphasize the need for education stakeholders to provide comprehensive supplemental resources to support gifted students of color. The educational experiences of these students are characterized by struggles with academic deficiency, underachievement and the careful navigation of sociocultural constructs that impede upon identity development. State, district and building-level personnel should consider the anecdotal stories shared by student peers and trusted adult informants as vital evidence pointing to the need for sociocultural supports to help promote positive self-efficacy and shape positive identity development.

I selected the study location, North High School, because of its central urban location and its diverse student population. North High is one of 100 schools throughout the state that adopted AdvanceState open enrollment protocols to increase AP access to low-income and students of color. The gifted African-American students who volunteered to participate in my study each identified an in-school peer and trusted adult 
informants that provided additional insight into student-informants' experiences. Studentinformants' overall perceptions of peers as influencing their efficacy beliefs varied, but all included acknowledgment of predominately African-American peer group identification, as well as positive, negative and neutral responses to both traditional and gifted peer interactions. All students gave themselves medium to high efficacy ratings when it came to completing AP coursework, however all had low confidence with regard to passing exams. Two of three student-informants identified sources of intrinsic motivation and one informant acknowledged codependency on peers to as a motivating factor. Moreover, student-informants cited the need for a support group designated specifically for students of color in AP classes in order to receive help with homework and connect with others about sociocultural issues.

In order to improve the effectiveness of initiatives like AdvanceState we must look beyond simply increasing diverse numbers of students in AP classrooms. Educators are responsible for ensuring that open-access nontraditional students are realizing the full benefits of gifted enrollment, including being prepared to pass cumulative AP exams to receive college credit. In order to address the academic struggles and sociocultural dynamics among gifted students of color, a component of open access initiatives should be a school-based holistic program that includes academic, familial and sociocultural resources and involves building-level stakeholders in various support roles. As educators we must be committed to maintaining an academic environment of excellence for all students, ensuring each individual receives an equitable education — which does not mean every child receives the same thing, but instead ensures each child has their individual needs met in order to be successful. A supplemental program that does not coddle 
students, but instead establishes expectations for high achievement, presents rigorous tasks, encourages parental involvement and fosters positive social relationships among teachers and peers would most certainly improve the educational experiences of gifted students of color. Perhaps if socioemotional woes have subsided and academic skills are enriched, then the concept of open access programs will not appear to be a wasted initiative after all. Meanwhile, as educators we must first recognize that gifted students come in many shades - from white to onyx. Second and most importantly, our gifted African-American students often navigate sociocultural issues and related peer pressures in silence, but we have the power to encourage, motivate, nurture and positively influence what they believe about themselves and quite possibly who they will become-but, we first must believe in them. 


\section{REFERENCES}

Adelman, C. (1999). Answers in the tool box academic intensity, attendance patterns, and bachelor's degree attainment. Washington, DC: U.S. Dept. of Education, Office of Educational Research and Improvement.

Ashforth, B., \& Mael, F. (1989). Social identity theory and the organization. Academy of Management Review. 14(1), 20-39.

Advancement Via Individual Determination: AVID. (2017). What is AVID?. Retrieved from http://www.avid.org/what-is-avid.ashx

Awaya, A. (2001). Equitable access to excellence: Opportunities for gifted education to an underrepresented population through open enrollment. Journal for the Education of the Gifted, 25(2), 177-197.

Baldwin, A. Y. (2004). Introduction to culturally diverse and underserved populations of gifted students. In A.Y. Baldwin (Ed.), Culturally diverse and underserved populations of gifted students (pp. xxiii-xxxi). Thousand Oaks, CA: Sage.

Bandura, A. (1977). Self-efficacy: toward a unifying theory of behavioral change. Psychological Review, 84(2), 191-215.

Bandura, A. (1982). Self-efficacy mechanism in human agency. American Psychologist, $37(2), 122-147$.

Bandura, A. (1986). Social foundations of thought and action: A social cognitive theory. Englewood Cliffs, NJ: Prentice-Hall.

Bandura, A. (1991). Social cognitive theory of self-regulation. Organizational Behavior and Human Decision Processes, 50(2), 248-287.

Bandura, A. (1993). Perceived self-efficacy in cognitive development and functioning. 
Educational Psychologist, 28, 117-148.

Bandura, A. (1997). Self-efficacy: The exercise of control. New York: W. H. Freeman.

Barber, B. L., Eccles, J. S., \& Stone, M. R. (2001). Whatever happened to the jock, the brain, and the princess? Young adult pathways linked to adolescent activity involvement and social identity. Journal of Adolescent Research, 16(5), 429-455.

Burke, M. A., \& Sass, T. R. (2013). Classroom peer effects and student achievement. Journal of Labor Economics, 31(1), 51-82.

Baxter, P., \& Jack, S. (2008). Qualitative case study methodology: Study design and implementation for novice researchers. The Qualitative Report, 13(4), 544-559.

Becker, B. E., \& Luthar, S. S. (2002). Social-emotional factors affecting achievement outcomes among disadvantaged students: Closing the achievement gap. Educational Psychologist, 37(4), 197-214.

Ben-Achour, Sabri. (February 20, 2013). More colleges stop giving credit for AP exams. Marketplace. Retreived from https://www.marketplace.org

Berndt, T. J., \& Keefe, K. (1995). Friends' influence on adolescents' adjustment to school. Child Development, 66(5), 1312-1329.

Berndt, T. J. (2002). Friendship quality and social development. Current Directions in Psychological Science, 11(1), 7-10.

Betancourt, J. R., Green, A. R., Carrillo, J. E., \& Ananeh-Firempong, O. (2003). Defining cultural competence: a practical framework for addressing racial/ethnic disparities in health and health care. Public Health Reports, 118(4), 293-302. 
Bong, M., \& Skaalvik, E.M. (2003). Academic self-concept and self-efficacy: How different are they really? Educational Psychology Review, 15, 1-40.

Bowen, G. A. (2009). Document analysis as a qualitative research method. Qualitative Research Journal, 9(2), 27-40.

Boyatzis, R. E. (1998). Transforming qualitative information: Thematic analysis and code development. Thousand Oaks, CA: Sage.

Britner, S. L., \& Pajares, F. (2006). Sources of science self-efficacy beliefs of middle school students. Journal of Research in Science Teaching, 43(5), 485-499.

Brooks-Gunn, J., \& Duncan, G. J. (1997). The effects of poverty on children. The Future of Children, 7(2), 55-71.

Callahan, C. (2003). Advanced placement and international baccalaureate programs for talented students in American high schools: A focus on science and mathematics. Retrieved from University of Connecticut, National Research Center on the Gifted and Talented. http://www.gifted.uconn.edu/nrcgt.html

Cairns, R. B., Cairns, B. D., \& Neckerman, H. J. (1989). Early school dropout: Configurations and determinants. Child Development, 60, 1437-1452.

Chappell, B. (January 17, 2013). AP Credit will no longer be accepted at Dartmouth. National Public Radio. Retrieved from http://www.npr.org

Chavous, T. M., Bernat, D. H., Schmeelk-Cone, K., Caldwell, C. H., Kohn-Wood, L., \& Zimmerman, M. A. (2003). Racial identity and academic attainment among African-American adolescents. Child Development, 74(4), 1076-1090.

Chen, X., French, D. C., \& Schneider, B. H. (Eds.). (2006). Peer relationships in cultural context. Cambridge: University Press. 
Chenoweth, K. (1998). The College Board decries preparation gap. Black Issues in Higher Education, 15(15), 24-25.

Cokley, K., McClain, S., Jones, M., \& Johnson, S. (2011). A preliminary investigation of academic disidentification, racial identity, and academic achievement among African America adolescents. The High School Journal, 95(2), 54-68.

Coleman, M., Gallagher, J., \& Foster, A. (1994). A Javits project: Gifted education policies program. Final report. (Research Report No. 143). University of North Carolina, Chapel Hill.

Corbin, J. M., \& Strauss, A. (2014). Basics of qualitative research: Techniques and procedures for developing grounded theory. Thousand Oaks, CA: Sage.

Creswell, J. (2009). Research design: Qualitative, quantitative, and mixed methods approaches (3rd ed.). Thousand Oaks, CA: Sage.

Creswell, J. W., \& Miller, D. L. (2000). Determining validity in qualitative inquiry. Theory into Practice, 39(3), 124-130.

Cruz, C. (2001). Toward an epistemology of a brown body. International Journal of Qualitative Studies in Education, 14(5), 657-669.

Cutcliffe, J., \& Mckenna, H. (1999). Establishing the credibility of qualitative research findings: The plot thickens. Journal of Advanced Nursing, 30(2), 374-380.

Darensbourg, A. M., \& Blake, J. J. (2014). Examining the academic achievement of black adolescents: Importance of peer and parental influences. Journal of Black Psychology, 40(2), 191-212. 
Darling-Hammond, L., \& Hill, P. T. (2015). Accountability and the federal role: A third way on ESEA. Retrieved from Stanford Center for Opportunity Policy in Education website https://edpolicy.stanford.edu/publications/pubs/1324

Davies, M., \& Kandel, D. B. (1982). Parental and peer influences on adolescents' educational plans: Some further evidence. American Journal of Sociology, 363387.

Denzin, N. K. (2009). The elephant in the living room: Or extending the conversation about the politics of evidence. Qualitative Research, 9(2), 139-160.

Dougherty, C., Mellor, L., \& Jian, S. (2006). The relationship between advanced placement and college graduation: 2005 AP study series (Report No. 1). Retrieved from National Center for Educational Accountability website http://www.nc4ea.org/index.cfm

Edwards, K., \& Duggan, O. (2012, July). Data-Based Decision Making: The road to AP equity. Paper presented at the annual meeting of the Advanced Placement Annual Conference (APAC). Retrieved from http://research.collegeboard.org

Eccles, J. S., Lord, S. E., Roeser, R. W., Barber, B. L., \& Jozefowicz, D. M. H. (1997). The association of school transitions in early adolescence with developmental trajectories through high school. In J. Schulenberg. J. L. Maggs \& K. Hurrelmann (Eds.), Health risks and developmental transitions during adolescence, (pp. 283$320)$.

Elementary and Secondary Education Act: Comparison of the No Child Left Behind Act to the Every Student Succeeds Act (2015). Retrieved from http://www.ascd.org 
Fielding, M. (2004a). 'New wave' student voice and the renewal of civic society. London Review of Education, 2(3), 197-217.

Fielding, M. (2004b). Transformative approaches to student voice: Theoretical underpinnings, recalcitrant realities. British Educational Research Journal, 30(2), 295-311.

Fielding, M., \& Rudduck, J. (2002, September). The transformative potential of student voice: confronting the power issues. In M. Fielding (Chair), Student consultation community and democratic tradition. Symposium conducted at the meeting of the annual conference of the British Educational Research Association, University of Exeter, England.

Flores-González, N. (1999). Puerto Rican high achievers: An example of ethnic and academic identity compatibility. Anthropology \& Education Quarterly, 30(3), $343-362$.

Flutter, J., \& Rudduck, J. (2004). Pupil consultation as a key to improving teaching and learning. In K. Myers \& J. Macbeath (Eds.), Consulting pupils: What's in it for schools? (pp. 1-21).

Flyvbjerg, B. (2006). Five misunderstandings about case-study research. Qualitative Inquiry, 12(2), 219-245.

Ford, D. Y. (1998). The under-representation of minority students in gifted education problems and promises in recruitment and retention. The Journal of Special Education, 32(1), 4-14. 
Ford, D. Y., \& Grantham, T. C. (2003). Providing access for culturally diverse gifted students: From deficit to dynamic thinking. Theory into Practice, 42(3), 217-225.

Ford, D.Y., Harris, J., \& Schuerger, J. (1993). Racial identity development among gifted black students: Counseling issues and concerns. Journal of Counseling \& Development, 71(4), 409-417.

Ford, D. Y., Harris III, J. J., Tyson, C. A., \& Trotman, M. F. (2001). Beyond deficit thinking: Providing access for gifted African-American students. Roeper Review, 24(2), 52-58.

Ford, D. Y. \& Thomas, A. (1997). Underachievement among gifted minority students: Problems and promises. Retrieved from https://www.ericdigests.org/1998-1/gifted.htm

Ford-Harris, D. Y., Schuerger, J. M., \& Harris, J. J. (1991). Meeting the psychological needs of gifted black students: A cultural perspective. Journal of Counseling and Development, 69, 577-580.

Fordham, S. (1988). Racelessness as a factor in Black students' school success: Pragmatic strategy or pyrrhic victory?. Harvard Educational Review, 58(1), 54-85.

Fordham, S., \& Ogbu, J. U. (1986). Black students' school success: Coping with the "burden of 'acting white'." The Urban Review, 18(3), 176-206.

Frasier, M. M, Garcia, J. H., \& Passow, A. H. (1995). A review of assessment issues in gifted education and their implications for identifying gifted minority students. 
Retrieved from University of Connecticut National Research Center on the Gifted and Talented website http://nrcgt.uconn.edu

Gallagher, S. A. (2009). Myth 19: Is advanced placement an adequate program for gifted students?. Gifted Child Quarterly, 53(4), 286-288.

Gibson, S., \& Dembo, M. H. (1984). Teacher efficacy: A construct validation. Journal of Educational Psychology, 76(4), 569.

Graham, A., \& Anderson, K.A. (2008). I have to be three steps ahead: Academically gifted African-American males students in an urban high school on the tension between ethnic and academic identity. Urban Review, 40, 472-499.

Graham, S. (1994). Motivation in Afro-Americans. Review of Educational Research, 64(1), 55-117.

Grantham, T. C., \& Ford, D. Y. (2003). Beyond self-concept and self-esteem: Racial identity and gifted African-American students. The High School Journal, 87, 1829.

Groenewald, T. (2004). A phenomenological research design illustrated. International Journal of Qualitative Methods, 3(1), 2-26.

Guay, F., Boivin, M. \& Hodges, E.V. (1999). Predicting change in academic achievement: A model of peer experiences and self-system processes. Journal of Educational Psychology, 91(1), 105-115 
Gubbins, E. J., Callahan, C. M., \& Renzulli, J. S. (2014). Contributions to the impact of the Javits Act by the national research center on the gifted and the talented. Journal of Advanced Academics, 25(4), 422-444.

Hanushek, E., Kain, F.F., Markman, J., \& Rivkin, S. (2003). Does peer ability affect student achievement? Journal of Applied Econometrics, 18(5), 527-544.

Haveman, R., \& Wolfe, B. (1995). The determinants of children's attainments: A review of methods and findings. Journal of Economic Literature, 33(4), 1829-1878.

Healy, M., \& Perry, C. (2000). Comprehensive criteria to judge validity and reliability of qualitative research within the realism paradigm. Qualitative Market Research: An International Journal, 3(3), 118-126.

Heidegger, M. (1977). Letter on humanism in basic writings. In D. Krell (Ed.), Basic Writings (pp. 189-242). New York, NY: Harper \& Row.

Hays, P. A. (2004). Case study research. In K. deMarrais \& S. Lapan (Eds.), Foundations for research: Methods of inquiry in education and the social sciences, (pp. 217234). Routledge.

Hibert, T. P. (2000). Defining belief in self: Intelligent young men in an urban high school. Gifted Child Quarterly, 44(2), 91-114.

Horvat, E. M., \& Lewis, K. S. (2003). Reassessing the" burden of 'acting white"': The importance of peer groups in managing academic success. Sociology of Education, 76(4), 265-280.

Hobbs, F., \& Stoops, N. (2002). Demographic trends in the 20th century. Washington, D.C.: Retrieved U.S. Census Bureau website from https://www.census.gov 
Howard, T. C. (2003). A tug of war for our minds: African-American high school students' perceptions of their academic identities and college aspirations. The High School Journal, 87(1), 4-17.

Hymel, S., Comfort, C., Schonert-Reichl, K., \& McDougall, P. (1996). Academic failure and school dropout: The influence of peers. In J. Juvonen \& K. R. Wentzel (Eds.), Social motivation: Understanding children's school adjustment (pp. 313-345).

Issues a-z: The Every Student Succeeds Act: An ESSA overview. (2016, March 31). Education Week. Retrieved from http://www.edweek.org/

Jencks, C., \& Phillips, M. (Eds.) (2011). The black-white test score gap. Washington, D.C.: Brookings Institution Press.

Kaiser, K. (2009). Protecting respondent confidentiality in qualitative research. Qualitative Health Research, 19(11), 1632-1641.

Kao, G., \& Thompson, J. (2003). Racial and ethnic stratification in educational achievement and attainment. Annual Review of Sociology, 29, 417-442.

Kaul, C., Johnsen, S., Witte, M., \& Saxon, T. (2015). Critical components of a summer enrichment program for urban low-income gifted students. Gifted Child Today, $38(1), 32-40$.

Kent, A. M. (2004). Improving teacher quality through professional development. Education, 124(3), 427-436.

Klopfenstein, K. (2004). Advanced placement: Do minorities have equal opportunity?. Economics of Education Review, 23(2), 115-131.

Kober, N. (2001). It takes more than testing: Closing the achievement gap. Center on Education Policy. Retrieved October 26, 2015 from http://www.ctredpol.org/ 
Kyburg, R. M., Hertberg-Davis, H., \& Callahan, C. M. (2007). Advanced placement and international baccalaureate programs: Optimal learning environments for talented minorities. Journal of Advanced Academics, 18(2), 172-215.

Ladson-Billings, G. (1999). Preparing teachers for diverse student populations: A critical race theory perspective. Review of Research in Education, 24, 211-247.

Ladson-Billings, G. (2006). From the achievement gap to the education debt: Understanding achievement in US schools. Educational Researcher, 35(7), 3-12.

LeCompte, M. D., \& Goetz, J. P. (1982). Problems of reliability and validity in ethnographic research. Review of Educational Research, 52(1), 31-60.

Lee, J. S., \& Bowen, N. K. (2006). Parent involvement, cultural capital, and the achievement gap among elementary school children. American Educational Research Journal, 43(2), 193-218.

Leech, B. L. (2002). Asking questions: Techniques for semi structured interviews. Political Science and Politics, 35(4), 665-668.

Leech, N. L., \& Onwuegbuzie, A. J. (2007). An array of qualitative data analysis tools: A call for data analysis triangulation. School Psychology Quarterly, 22(4), 557-84.

Lester, S. (1999). An introduction to phenomenological research. Retrieved from https://www.researchgate.net/publication/255647619/

Lichten, W. (2007). Equity and excellence in the College Board advanced placement program. The Teachers College Record, 6(7).

Marland, S. P., Jr. (1971). Education of the gifted and talented-volume 1: Report to the Congress of the United States by the U.S. Commissioner of Education. 
Marshall, H. W., \& Weinstein, R. S. (1984). Classroom factors affecting students' selfevaluations: An interactional model. Review of Educational Research, 54(3), 301325.

Mathison, S. (1988). Why Triangulate? Educational Researcher, 17(2), 13-17.

McCoach, D. B., \& Siegle, D. (2003). Factors that differentiate underachieving gifted students from high-achieving gifted students. Gifted Child Quarterly, 47(2), 144154.

Merriam, S. (2002). Introduction to qualitative research. In S. Merriam (Ed.), Qualitative research in practice: Examples for discussion and analysis (pp. 1-17). San Francisco, CA: Josey-Bass.

Miles, M. B., \& Huberman, A. M. (1994). Qualitative data analysis: An expanded sourcebook (2nd ed.). Thousand Oaks, CA: Sage.

Miles, M. B., Huberman, A. M., \& Saldaña, J. (2014). Qualitative data analysis (3rd ed.). Thousand Oaks, CA: Sage.

Milner, H.R. (2007). Race, culture, and researcher positionality: Working through dangers seen, unseen, and unforeseen. Educational Researcher, 36(7), 388-400.

Mitra, D. (2004). The significance of students: can increasing "student voice" in schools lead to gains in youth development?. Teachers College Record, 106(4), 651-688.

Mitra, D., Serriere, S., \& Stoicovy, D. (2012). The role of leaders in enabling student voice. Management in Education, 26(3), 104-112.

Moustakas, C. (1994). Phenomenological research methods. Thousand Oaks, CA: Sage. 
National Association for Gifted Children. (2015a, October 8). Why are gifted programs needed?.

National Association for Gifted Children. (2015b, December 29). Definitions for giftedness. Retrieved from NAGC website https://www.nagc.org/resourcespublications/resources/definitions-giftedness

National Association for Gifted Children. (2016, February 28). Questions and answers about every student succeeds act.

Ndura, E., Robinson, M., \& Ochs, G. (2003). Minority students in high school Advanced Placement courses: Opportunity and equity denied. American Secondary Education, 32(1), 21-38.

Nelson, R. M., \& DeBacker, T. K. (2008). Achievement motivation in adolescents: The role of peer climate and best friends. The Journal of Experimental Education, 76(2), 170-189.

Noor, K. B. M. (2008). Case study: A strategic research methodology. American Journal of Applied Sciences, 5(11), 1602-1604.

Oakes, J. (1990). Multiplying inequalities: The effects of race, social class, and tracking on opportunities to learn mathematics and science. Santa Monica, CA: The RAND Corporation.

Ogbu, J. U. (1991). Minority coping responses and school experience. Journal of Psychohistory, 18(4), 433-456.

Oldfather, P. (1995). This issue: Learning from student voices. Theory into Practice, 34(2), 86-87. 
Oldfather, P., \& Thomas, S. (1998). What does it mean when high school teachers participate in collaborative research with students on literacy motivations?. Teachers College Record, 99(4), 647-91.

Olszewski-Kubilius, P. (2006). Addressing the achievement gap between minority and nonminority children: Increasing access and achievement through project EXCITE. Gifted Child Today, 29(2), 28-37.

Olszewski-Kubilius, P., Lee, S., Ngoi, M., \& Ngoi, D. (2004). Addressing the achievement gap between minority and nonminority children by increasing access to gifted programs. Journal for the Education of the Gifted, 28(2), 127-158.

Oyserman, D., Harrison, K., \& Bybee, D. (2001). Can racial identity be promotive of academic efficacy?. International Journal of Behavioral Development, 25(4), 379-385.

Pajares, F. (1996). Self-efficacy beliefs in academic settings. Review of Educational Research, 66, 533-578.

Pajares, F. (2003). Self-efficacy beliefs, motivation, and achievement in writing: A review of the literature. Reading \&Writing Quarterly, 19(2), 139-158.

Paris, D. (2012). Culturally sustaining pedagogy: A needed change in stance, terminology, and practice. Educational Researcher, 41(3), 93-97.

Patton, M. Q. (1990). Qualitative research and evaluation methods. Thousand Oaks, CA: Sage Publications.

Patton, M. Q. (2002). Qualitative research and evaluation methods. Thousand Oaks, CA: Sage Publications.

Pintrich, P. R., \& Schunk D. H. (1996). Motivation in education: Theory, research, 
and applications. Englewood Cliffs, NJ: Merrill/Prentice Hall.

Reis, S. M., Hibert, T. P., Diaz, E. I., Maxfield, L. R., \& Ratley, M. E. (1995). Case studies of talented students who achieve and underachieve in an urban high school (Research Monograph No. 95120). Retrieved from National Research Center on the Gifted and Talented website http://nrcgt.uconn.edu/researchbased_resources/reishebe/

Renzulli, J. S., Callahan, C. M., \& Gubbins, E. J. (2014). Laying the base for the future one cornerstone of the Javits Act. Journal of Advanced Academics, 25(4), 338348.

Ritchie, J., Lewis, J., Nicholls, C. M., \& Ormston, R. (Eds.). (2013). Qualitative research practice: A guide for social science students and researchers. Thousand Oaks, CA: Sage.

Robertson, D., \& Symons, J. (2003). Do peer groups matter? Peer group versus schooling effects on academic attainment. Economica, 70(277), 31-53.

Rodgers, K.A. (2008). Racial identity, centrality and giftedness: An expectancy-value application of motivation gifted African-American students. Roeper Review, (30), 111-120.

Roeser, R. W., \& Lau, S. (2002). On academic identity formation in middle school settings during early adolescence. In T. Brinthaupt \& R. Lipka (Eds.), Understanding early adolescent self and identity: Applications and interventions (pp. 91-131). Albany: SUNY. 
Rosenholtz, S. J., \& Simpson, C. (1984). The formation of ability conceptions: Developmental trend or social construction?. Review of Educational Research, 54(1), 31-63.

Ross, P. (1993). National excellence: A case for developing America's talent: An anthology of readings. Office of Educational Research and Improvement, Washington, D.C.

Rudduck, J., \& Flutter, J. (2000). Pupil participation and pupil perspective: 'Carving a new order of experience'. Cambridge Journal of Education, 30(1), 75-89.

Ryan, A.R. (2000). Peer groups as a context for the socialization of adolescents' motivation, engagement, and achievement in school. Educational Psychologist, 35(2), 101-111.

Saldana, J. (2013). The coding manual for qualitative researchers (2nd ed.). Thousand Oaks, CA: Sage.

Schunk, D. H., \& Hanson, A. R. (1985). Peer models: Influence on children's selfefficacy and achievement. Journal of Educational Psychology, 77(3), 313.

Schunk, D. H., Hanson, A. R., \& Cox, P. D. (1987). Peer-model attributes and children's achievement behaviors. Journal of Educational Psychology, 79, 54-61.

Schunk, D. H. (1991). Self-efficacy and academic motivation. Educational Psychologist, 26(3-4), 207-231.

Schunk, D. H. (1995). Self-efficacy and education and instruction. In J. E. Maddux (Ed.), Self-efficacy, adaptation, and adjustment: Theory, research, and application (pp. 281-303). New York: Plenum Press. 
Schunk, D.H., \& Pajares, F. (2001). The development of academic self-efficacy. In A. Wigfield \& J. Eccles (Eds.), Development of Achievement Motivation. San Diego: Academic Press.

Schunk, D. H., \& Swartz, C. W. (1993). Goals and progress feedback: Effects on selfefficacy and writing achievement. Contemporary Educational Psychology, 18(3), $337-354$.

Siegle, D. (2001). Overcoming bias in gifted and talented referrals. Gifted Education Communicator, 32(2), 22-25.

Smith, E. M. J. (1989). Black racial identity development: Issues and concerns. The Counseling Psychologist, 17(2), 277-288.

Sokolowski, R. (2000). Introduction to phenomenology. Cambridge: University Press.

Solorzano, D.G., \& Ornelas, A. (2004). A critical race analysis of Latina/o and AfricanAmerican Advanced Placement enrollment in public high schools. The High School Journal, 87(3), 15-26.

Speroni, C. (2011). Determinants of students' success: The role of Advanced Placement and dual enrollment programs (NCPR Working Paper). New York, NY: National Center for Postsecondary Research.

Stake, R. E. (1978). The case study method in social inquiry. Educational Researcher, $7(2), 5-8$

Stake, R. E. (1995). The art of case study research. Thousand Oaks: Sage Publications.

Stake, R. E. (2005). Qualitative case studies. In N. K. Denzin \& Y. S. Lincoln (Eds.), Qualitative Research (3rd ed., pp. 443-466). Thousand Oaks, CA: Sage. 
Stallworth, C.A., \& Thomas, K.D. (2017). Minority recruitment and retention among gifted students. In M. Gray \& K. Thomas (Eds.), Strategies for increasing diversity in engineering majors and careers, (p. 26-59). IGI Global.

Steele, C., Spencer, S., \& Aronson, J. (2002). Contending with group image: The psychology of stereotype and social identity threat. Advances in Experimental Social Psychology, 34, 379-440.

Steinberg, L., Brown, B., \& Dornbusch, S. (1996). The real problem. In Beyond the classroom: Why school reform has failed and what parents need to do (pp. 1328). New York, NY: Simon \& Schuster.

Stephens, K. R., \& Karnes, F. A. (2000). State definitions for the gifted and talented revisited. Exceptional Children, 66(2), 219-238.

Strauss, A., \& Corbin, J. (1990). Basics of qualitative research (Vol. 15). Newbury Park, CA: Sage.

Sumsion, J. (2002). Becoming, being and unbecoming an early childhood educator: A phenomenological case study of teacher attrition. Teaching and Teacher Education, 18(7), 869-885.

Tajfel, H., \& Turner, J.C. (1986). The social identity theory of intergroup behavior. In S. Worchel \& W.G. Austin (Eds.), The psychology of intergroup relations (pp. 724).

Tang, S., McLoyd, V.C., Hallman, S.K. (2016). Racial socialization, racial identity, and academic attitudes among African-American adolescents: Examining the moderating influence of parent-adolescent communication. Journal of Youth Adolescence, 45, 1141-1155. 
Teachman, J., Day, R., Paasch, K., Carver, K., \& Call, V. (1998). Sibling resemblance in behavioral and cognitive outcomes: The role of father presence. Journal of Marriage and the Family, 60(4), 835-848.

The College Board. (2001). Equity 2000: A systematic education reform model: A summary report 1990-2000. Retrieved from http://www.collegeboard.com/prod_downloads/about/association/equity The College Board. (2003). A brief history of the Advanced Placement program. Retrieved from http://www.collegeboard.com/prod_downloads/about/news_info/ap/ap_history The College Board. (2006). National summary reports 2006 [Electronic version]. Retrieved from http://www.collegeboard.com/student/testing/ap/exgrd_sum/2006.html

The College Board. (2015, November 28). AP Exams. Retrieved from http://apcentral.collegeboard.com/apc/public/exam/index.html

The Princeton Review. (2017). All about your AP scores. Retrieved from https://www.princetonreview.com/college-advice/ap-scores

The White House, Office of Press Secretary. (2015, December 2). Fact sheet: Congress acts to fix No Child Left Behind. Retrieved from https://www.whitehouse.gov/thepress-office/2015/12/03/fact-sheet-congress-acts-fix-no-child-left-behind Trochim, W. (2006, October 20). Qualitative Validity. In The Research Methods Knowledge Base (2nd ed.). Retrieved from http://www.socialresearchmethods.net/kb/qualval.php 
Usher, E. L. (2009). Sources of middle school students' self-efficacy in mathematics: A qualitative investigation of student, teacher, and parent perspectives. American Educational Research Journal, 46, 275-314.

Usher, E. L., \& Pajares, F. (2006). Sources of academic and self-regulatory efficacy beliefs of entering middle school students. Contemporary Educational Psychology, 31(2), 125-141.

U.S. Department of Education, National Center for Education Statistics. (2009a). Achievement gaps: How Hispanic and White students in public high schools perform in mathematics and reading on the National Assessment of Educational Progress. [Data file]. Retrieved from http://nces.ed.gov/nationsreportcard/studies/gaps/hwgaps_press.aspx

U.S. Department of Education, National Center for Education Statistics. (2009b). Achievement gaps: How Black and White students in public high schools perform in mathematics and reading on the National Assessment of Educational Progress. [Data file]. Retrieved from http://nces.ed.gov/nationsreportcard/pubs/studies/2009455.asp

U.S. Department of Education, National Center for Education Statistics. (2010). Status and trends in the education of racial and ethnic groups. [Data file]. Retrieved from http://nces.ed.gov/pubs2010/2010015

U.S. Department of Education, National Center for Education Statistics. (2016). Digest of education statistics, 2015 (NCES 2016-014). Retrieved from https://nces.ed.gov/fastfacts/display.asp?id=98 
Van Tassel-Baska, J., Feng, A.X., Swanson, J.D., Quek, C. \& Chandler, K. (2009). Academic and affective profiles of low-income, minority, and twice exceptional gifted learners: The role of gifted program membership in enhancing self. Journal of Advanced Academics, 20(4), 702-739.

Viadero, D., \& Johnston, R. C. (2000). Lags in minority achievement defy traditional explanations. The achievement gap. Education Week, 19(28), 18-22.

Walker, C. O., \& Greene, B. A. (2009). The relations between student motivational beliefs and cognitive engagement in high school. The Journal of Educational Research, 102(6), 463-472.

Wentzel, K. R. (1991). Social competence at school: Relation between social responsibility and academic achievement. Review of Educational Research, 61(1), $1-24$.

Wertz, F. (2005). Phenomenological research methods for counseling psychology. Journal of Counseling Psychology, 52(2), 167-177.

West, E. T. (2013). A phenomenological case study of the experiences of AfricanAmerican high school students. Sage Open, 3(2), 1-11.

White, K. R. (1982). The relation between socioeconomic status and academic achievement. Psychological Bulletin, 91(3), 461-481.

Winebrenner, S. (2006). Effective teaching strategies for open enrollment honors and AP classes. Journal of Secondary Gifted Education, 17(3), 159-177.

Yin, R. (1984) Case study research. Beverly Hills, CA: Sage Publications. 
Yin, R. K. (2003). Case study research: Design and methods (3rd ed.). Thousand Oaks, CA: Sage.

Yin, R. (2009). How to do better case studies. In L. Bickman \& D. J. Rog (Eds.), The Sage Handbook of Applied Social Research Methods (2nd ed., pp. 254-282). Thousand Oaks, CA: Sage.

Zarate, M. E., \& Pachon, H. P. (2006). Gaining or losing ground? Equity in offering Advanced Placement courses in California high schools 1997-2003. Retrieved http://trpi.org

Zeldin, A. L., \& Pajares, F. (2000). Against the odds: Self-efficacy beliefs of women in mathematical, scientific, and technological careers. American Educational Research Journal, 37(1), 215-246.

Zimmerman, B. J. (1990). Self-regulated learning and academic achievement: An overview. Educational Psychologist, 25(1), 3-17.

Zimmerman, D.J. (2003). Peer effects in academic outcomes: Evidence from a natural experiment. The Review of Economics and Statistics, 85(1), 9-23. 


\section{APPENDIX A}

Student Interview Protocol (semi-structured)

Adapted from Usher (2009), originally modified from Zeldin and Pajares (2000)

\section{Background}

1. Tell me about the schools you have previously attended.

a. How long have you been at this school?

2. Tell me a little bit about your family.

3. Tell me about yourself.

a. What is your personality like?

b. Tell me about your friends.

c. Do you enjoy school? (explain)

d. Who do you most admire? Tell me about him/her/them.

4. Describe yourself as a student

a. What is your best subject? Why?

b. What is your favorite subject? Why?

c. Which subject is your weakest? Why?

d. Which subject is your least favorite? Why?

5. Tell me about your academic grades. Do you feel your grades truly reflect your understanding/knowledge in the courses? Why?

Advance Placement Experiences and Self-Efficacy

I am going to ask you several questions about your social and academic experiences as an AP student.

6. If you were asked to rate your (potential to succeed) overall ability in your AP courses on a scale of 1 (lowest) to 10 (highest), where would you be? Why?

7. Using the same scale, rate your confidence in doing AP work. Why? What could make you feel more confident about your work?

8. Using the same scale, rate your confidence in passing the AP exam(s) at the end of the year. Have you passed AP exams in the past? If so, how many and which courses? What could make you feel more confident about taking AP exams?

9. Tell me about a time you experienced a setback in your AP class(es). How did you deal with it?

10. How do you handle difficult assignments/tasks in your AP classes?

11. Do you consider yourself to be self-motivated? Example? 


\section{Relationships \& Academic Identity}

I want you to think about all of the AP classes you have taken and your experiences with AP and non-AP peers. I am going to ask you some questions about your experiences with these peers.

12. Would you say the majority of your friends are AP or non-AP students? Why?

13. Has this always been the case?

a. Do your friends belong to a particular race or ethnicity? Why? Do you have non-AP friends support your decision to be in AP?

14. On average, how many minority students are in your AP courses?

15. Do your friends (not necessarily your classmates) support your choice to take AP classes? Why?

16. Describe how your most of your friends do in AP classes.

b. How do they handle difficult assignments/tasks?

17. What do your friends say about AP classes? What do they say about those who do well in AP?

18. How do your friends/peers feel about your choice to take AP courses?

19. How do you think your AP peers would describe how you "act" as a result of your enrollment in AP classes?

20. How would your non-AP peers describe how you "act" as a result of your enrollment in AP classes?

21. Is there anything else you'd like to tell me about your experiences with your peers and non-AP peers?

\section{Academic Identity}

Now, I want you to think about how you identify/describe yourself as a student, including how those around you might describe you. How you describe yourself as a student is referred to as 'academic identity."

22. Describe your experience as an AP student.

a. How long have you been enrolled in AP classes?

b. Currently, how many AP classes are you enrolled in? What subject $\operatorname{area}(\mathrm{s})$ ?

c. Tell about your academic experiences in AP classes.

i. Describe some of the positive experiences you have had as an AP student?

ii. Describe any negative experiences you have encountered as an $n$ AP student.

d. Tell me about your nonacademic experiences in AP classes (e.g., social, cultural)

23. What qualities best describe you as a student? 
24. Do your friends in AP classes influence how you identify yourself as a student? Explain.

25. Do your non-AP friends influence how you identify yourself as a student? Explain.

26. What concerns you the most about being an AP student? Least? 


\section{APPENDIX B}

Trusted Adult Interview Protocol (semi-structured)

\section{Acquaintance}

1. What is your role/position at North High School?

2. How long have you been employed in this district? At NHS?

3. How long have you known (participant name)?

4. How did you come to know (participant name)?

5. How would you describe your relationship with (participant name)

Perception of Academic Identity

1. How would you describe (participant name) as a student?

2. Do you believe he/she exhibits qualities of an AP student? (e.g., organized, selfmotivated, academic focus) Explain.

Perception of Peer Relationships

1. Describe an occasion which you have observed this student interacting with AP classmates?

2. Describe an occasion which you have observed this student interacting with non AP classmates?

3. To what extent do you believe (participant name) peers influence his/her academic progress? 


\section{APPENDIX C}

\section{Peer Interview Protocol (semi-structured)}

\section{Acquaintance}

1. How long have you been a student at NHS?

2. How long have you known (participant name)?

3. How did you come to know (participant name)?

4. Do you currently or have you had classes with (participant name)?

5. How would you describe your relationship with (participant name)

Perception of Academic Identity

1. How would you describe (participant name) as a student?

2. Do you believe he/she exhibits qualities of an AP student? (e.g., organized, selfmotivated, academic focus) Explain.

Perception of Peer Relationships

1. Describe an occasion which you have observed this student interacting with AP classmates?

2. Describe an occasion which you have observed this student interacting with non AP classmates?

3. To what extent do you believe (participant name) peers influence his/her academic progress? 


\title{
CURRICULUM VITAE
}

\author{
Tinisha Y. Taylor
}

\section{CAREER OBJECTIVE}

To obtain a counseling position at an educational institution that embraces the diversity of its students and staff, places student growth as a priority and values whole-child education.

EDUCATION

EARNED

EdD Professional Doctorate (EdD)

August 2017 (expected)

Educational Leadership and Organizational Development

University of Louisville-Louisville, KY

MEd University of Louisville - Louisville, KY 2010

MAT University of Louisville - Louisville, KY 2005

BA University of Louisville - Louisville, KY 2001

\section{CERTIFICATIONS}

Kentucky Professional Certification - Biology Grades 8-12

EXPIRES

Kentucky Certificate - School Counseling All Grades

\section{PROFESSIONAL EXPERIENCE}

April 2016-June 2017

Valley Preparatory Academy

Louisville, $\mathrm{KY}$

Professional School Counselor

- Assists with master scheduling for eighth grade

- Maintains and auditing student educational records

- Leads classroom guidance lesson for academic planning and social-emotional progression

- Positive Behavior Intervention and Supports Lead

- Manages eighth grade transition center; conferences regularly with students and parents/guardians regarding progress and program exit expectations.

- Creates and modifies student schedules and course rosters to ensure students are placed in the most appropriate instructional setting

- Collaborates with mental health counselor for mental health triage and support

- Facilitates individual small group guidance for students to address socialemotional issues

- Communicates with families to provide access to education and community resources and assist with academic planning

- Organizes community outreach programs to promote student success

- Conferences with students and families to improve academic performance, attendance and/or behavior

- Administrator assigned to Science Department and Related Arts PLC

- Manages special education services for students adhering to federal, state and district policies, chairing Admissions and Release Committee (ARC) and 504

Plan meetings and develops data collection systems for teachers.

- Assists with referrals for optional magnet and alternative programs

- Student supervision in school common areas and/or classrooms 
- Performs other duties as assigned by the principal.

\author{
January 2016-April 2016 \\ Valley Preparatory Academy \\ Behavior Coach / Resource Teacher / PBIS Lead \\ Louisville, $\mathrm{KY}$ \\ - Responded to classroom disruptions to deescalate and redirect student behaviors \\ as part of the Student Response Team (SRT). \\ - Led Positive Behavior Interventions and Supports (P.B.I.S.) Team; facilitate \\ meetings and trainings \\ - Designed and facilitated professional development training sessions for school \\ staff on implementation of P.B.I.S. principles \\ - Collaborated with team members to develop student expectations for school \\ common areas \\ - Attended nationally accredited P.B.I.S. training sessions; analyzes behavior data \\ and develops school-wide action plan \\ - Conducted classroom and common area observations to ensure P.B.I.S. \\ principles are implemented with fidelity and provide feedback to staff \\ - Collaborated with teachers, parents and students to create behavior intervention \\ plans for students to provide support and strategies for self-managed behavior \\ - Facilitated professional development training for teachers on classroom \\ management strategies \\ - Conducted classroom and common area observations to assess classroom \\ management protocols and strategies and provide feedback and resources \\ - Attended Safe Crisis Management training and follow up training \\ - Engaged in individual and group counseling with students to help build self- \\ esteem, social skills and self-manage their behavior \\ - Assigned tiered-level behavior resolutions for disciplinary referrals \\ - Facilitated peer mediation sessions to resolve student-student issues \\ - Assisted administration and support staff with supervision of common areas \\ - Other duties as assigned by the principal
}

\title{
August 2015-December 2016
}

Science Department Chairperson

\section{Seneca High School Louisville, KY}

- Evaluated the department program and assists the Principal in evaluating the curriculum.

- Consulted with the Principal on problems of the department and provides leadership to the staff development process.

- Worked cooperatively with other department chairpersons to diagnose instructional and management needs of the faculty

- Developed workshops and professional development training designed to meet the needs of the faculty

- Worked with Principal and department members to effect a peer coaching process to ensure effective teaching strategies designed to positively impact the learning process within the department; observes and assists teachers upon request of the teacher or Principal.

- Represented the department in meetings to evaluate programs, develop procedures and recommend policies.

- Advised and informs Principal of system wide progress, procedures and policies related to the department and assists in their implementation. 
- Assisted in preparation of the departmental budget and in requisitioning materials.

- Worked with Academic Sponsor to identify and train students for academic competition.

- New teachers orientation and assisted with materials, records and reports.

- Performed other duties as assigned by the principal.

August 2015-December 2016 Seneca High School

Louisville, $\mathrm{KY}$

Positive Behavior Interventions and Supports (P.B.I.S.) Team/Secretary

- Created meeting agendas and distributes to team members

- Participated in team meetings and assists members in analyzing, evaluating and implementing P.B.I.S. framework throughout the school

- Maintained accurate record of meeting minutes and distributes to team members and administrators

- Designed and facilitates professional development training sessions for school staff on implementation of P.B.I.S. principles

- Collaborated with team members to develop student expectations for school common areas

- Attended nationally accredited P.B.I.S. training sessions

- Conducted classroom and common area observations to ensure P.B.I.S. principles are implemented with fidelity and provide feedback to staff

\section{September 2015-December $2016 \quad$ Seneca High School Louisville, KY}

Extended School Services Coordinator (Winter School, Summer School)

- Developed ESS logistics plan for students, teachers, staff and administrative supervision and submits to principal for approval

- Established expectations for student academic progress (credit recovery) and behavior

- Collaborated with administration to coordinate before/after ESS bus transportation

- Coordinated with administrators and cafeteria manger to arrange complimentary meals for students

- Distributed, collects and submits payroll information for ESS staff

- Maintained contact with parents/guardians regarding ESS student attendance and discipline concerns

- Worked with counseling staff to ensure students recovered credits are allocated

\section{August 2014-December 2016 Seneca High School Louisville, KY}

Science Teacher (Biology), Technology Focus Team, Junior/Senior Class Sponsor

- Met and instructs assigned classes in the locations and at the times designated.

- Instructor for Honors and Advanced (Pre-AP) Biology courses

- Created and maintains a classroom environment that is conducive to learning and appropriate to the maturity and interests of students.

- Collaborated with PLC to analyze assessment data, revise instruction and develop common formative assessments

- Guided the learning process toward the achievement of curriculum goals 
- Established objectives for all lessons, units, projects, and the like in order to communicate these objectives to students.

- Attended staff meetings, serves on staff committees, and accepts a share of responsibility for extracurricular activities.

- Developed reasonable rules of classroom behavior and procedure, and maintains order in the classroom in a fair and just manner Communicates achievement results and academic progress to parents, students and instructional team

- Facilitated faculty learning opportunities on use of technology in the classroom

- Maintained accurate, complete and correct records as required by law, district policy, and administrative regulation.

- Employed instructional methods and materials that are appropriate for meeting stated objectives, prepares for classes

- Participated on Technology Focus Team and "Bring Your Own Device" Cohort

- Developed and implement individualized web-based learning opportunities for Integrated Science1A/1B, Biology

- Engaged in parent/teacher conferences as necessary to assist the parent's participation and support of a child's education.

- Continued personal professional growth and upgrading of skills appropriate to teaching assignments.

- Assessed the accomplishments of students on a regular basis and provides progress reports and counseling to parents as required concerning academic and behavioral progress of all assigned students.

- Organized, plans and coordinates junior/senior class events

- Performed other duties as assigned by the principal or school center head.

August 2005-May $2014 \quad$ Southern High School MCA Louisville, KY Science Teacher (Biology, Chemistry, Physics)

Professional Learning Community Lead

- Instructed Integrated Science (Chemistry and Physics), Biology, Forensic Science, Human Anatomy

- Planned and design inquiry instruction based on National Science Standards

- Facilitated Professional Learning Community (PLC) meetings

- Collaborated with PLC to develop curriculum, analyze instruction, develop common formative assessments

- Collected formative assessment data and analyze results to plan/revise lessons

- Communicated with parents/guardians, work alongside to create appropriate intervention strategies for students

- Developed/Implemented individualized web-based learning opportunities for Integrated Science 1A/1B, Biology, Forensics, Human Anatomy

- Cooperated with administrators and counselors to address student barriers to success

- Supervised students in the classroom, hallways and on field trips, manage daily classroom activities/behavior

- Performed other duties as assigned by the principal 


\section{School Counselor Intern}

Worked with students to plan yearly class schedules

- Oversaw the completion of student individual learning plans

- Met with parents/guardians to evaluate student progress

- Planned and led classroom guidance sessions

- Created student schedules in infinite campus

- Assisted in supervision of standardized testing

- Counseled students on personal/individual concerns

- Implemented small group sessions with at-risk students to improve achievement

- Planed fundraiser for family resource center

- Performed other duties as assigned by the principal or counselor mentor

\section{RELATED WORK \& LEADERSHIP EXPERIENCE}

\section{August 2005 - May $2014 \quad$ Southern High School MCA}

Louisville, $\mathrm{KY}$

Future Educators Association Chapter Advisor

- Facilitated educational workshops at local and national conferences

- Recruited/Supported diverse groups of students for chapter membership

- Plans chapter and district fundraising events

- Conducted weekly chapter meetings with students

- Collaborated with teachers and administrators at elementary school to assess student needs and created high school mentor/tutor programs

- Coordinated group large and small community service projects

- Managed and planned group travel for school and district chapters

- Assisted with designing and planning district and state conferences

- Supervised award-winning students to compete in district, state, regional and national conferences

- Chartered FEA chapter at T.T. Knight Middle school and mentorship program with advisors and students Knight Middle

August 2013-May 2014

PTSA Vice-President Membership

Louisville, $\mathrm{KY}$

Southern High School MCA

- Recruited parents, teachers and staff for membership

- Assisted with recruitment of parent volunteers

- Organized membership drive and related events

- Distributed local, state and national membership information and benefits

- Planned and facilitated fundraising events

- Attended National PTA conference; membership training, workshops

- Facilitated chapter meetings 
August 2011-May 2015 Kentucky Teacher Internship Program Resource Teacher Louisville, KY Southern High School MCA/Seneca High School

- Served as primary mentor for teacher intern

- Assisted teacher intern with lesson planning and differentiation and classroom management techniques

- Observed and evaluate teacher intern during class to ensure state standards are met

- Coordinated pre/post-evaluation meetings with teacher intern to evaluate progress and provide feedback

- Participated in KTIP committee meetings to asses teacher intern's progress

- Performed other duties as assigned by KTIP administrator

\section{August 2012-December 2015 Student Teacher Supervisor Louisville, KY}

Southern High School MCA / Seneca High School / University of Louisville

- Collaborated with student teacher to design and develop lesson plans

- Observed and records student teacher instruction

- Counseled student teacher on instruction reflection

- Mentored student teacher on classroom management techniques

August-May $2013 \quad$ Freshman Academy Leadership Team Louisville, KY Southern High School MCA Facilitated weekly Freshman Academy Team meetings

- Attended professional development seminars for improving academy structure

- Developed programs to assist with transitional issues

- Met with team teachers to evaluate student progress, plan activities and develop student interventions

- Planned inaugural freshman pledge ceremony where students commit to academic success and graduation

- Evaluated academic progress for ninth grade students

- Developed celebration programs and events to encourage and support student success

August 2009-May 2013; August 2011-May 2012

Professional Learning Community Leader Louisville, KY Southern High School

- Facilitated meetings with colleagues

- Collaborated to develop realistic, rigorous, relevant formative and summative assessments based on National Science Standards

- Analyzed/Interpreted assessment data to guide planning and instruction

- Assisted with planning of freshman pledge ceremony and designed banner and commitment card

- Created watch list of students and developed intervention strategies to address issues of attendance, behavior and academics 


\section{August 2012 - May 2014 Leadership Goal Team-School Culture Louisville, KY}

Southern High School MCA

- Collaborated with teachers to review CSIP on school culture

- Planned strategies to improve school culture through various methods

- Focus on improve student attendance and behavior

- Listened to input from student representatives regarding school culture

- Revise CSIP to reflect new goals/improvements in school culture

\section{Professional Learning Community Leader (Biology) Louisville, KY}

\section{Southern High School MCA}

- Collaborated with regular education and exceptional education teachers to create differentiated lessons for students of all levels

- Developed formative and summative assessments based on core content standards

- Implemented inquiry-based instruction enhancing student engagement

- Designed effective tools to communicate achievement results to students

- Created cumulative projects and research experiences to solidify content comprehension

- Modified inquiry-based activities to address student needs

- Analyzed student data for summary distribution to colleagues and administrators

- Facilitated scheduled weekly meetings

- Performed other duties as assigned by resource teacher and administrator

August 2009 - May 2010

CIMS Chemistry Program

Professional development for non-chemistry certified teachers to enhance their knowledge of general chemistry through simulated, student-centered, hands-on inquiry based learning

\section{AWARDS \& RECOGNITIONS}

FEA/MTRP Outstanding Chapter Advisor (2007, 2008, 2009, 2012)

JCPS Superstar Volunteer Award (2012)

Counselors for New Millennium Scholarship Recipient- University of Louisville (2007-10)

Dr. William C. Parker Scholarship Recipient- University of Kentucky (1996)

\section{PROFESSIONAL AFFLIATIONS}

JCPS Women in Administration

Jefferson County Association of School Administrators

Jefferson County Counselors Association

American School Counselors Association (ASCA)

Kentucky Counselors Association (KCA)

Kentucky Education Association (KEA)

Zeta Phi Beta Sorority, Inc.

Greater Louisville Alliance of Black School Educators (GLASBE)

Phi Delta Kappa International / Future Educators Association (PDK / FEA)

Multicultural Teacher Recruitment Program (MTRP) 